\section{Check for updates}

Cite this: Mater. Adv., 2021, 2, 1927

Received 31st December 2020, Accepted 24th January 2021

DOI: 10.1039/d0ma01030h

rsc.li/materials-advances

\title{
Progress of hybrid nanocomposite materials for thermoelectric applications
}

\author{
Neha Bisht, ${ }^{a}$ Priyesh More, (D)*a Pawan Kumar Khanna, (D) *a Reza Abolhassani, (DD ${ }^{b}$ \\ Yogendra Kumar Mishra (D) $^{\mathrm{b}}$ and Morten Madsen (D) ${ }^{\mathrm{b}}$
}

\begin{abstract}
Presently, the energy crisis and environmental burden have become two major problems due to the mismanagement of thermal power. Accordingly, the tremendous amount of waste heat from various energy sectors can be utilized by thermoelectric generators as a green energy source. Generally, thermoelectric generators (TEGs) exhibit enormous potential for converting waste heat to electricity without involving any mechanical vibrations and noises. However, they suffer from the disadvantages of expensive manufacturing techniques, low reliability, and scarce availability of conventional thermoelectric materials. Therefore, there has been significant interest from researchers in developing advanced new thermoelectric materials (TEMs). Recently, the promising thermoelectric properties of organic and inorganic nanomaterial-based hybrid nanocomposites have gained popularity among researchers. The grafting of conducting organic and inorganic thermoelectric nanoparticles on graphene/CNT matrices combines the advantages of both inorganic and organic materials. The present review comprehensively presents new hybrid nanocomposite-based TEMs used specifically for wearable TEGs. To demonstrate the performance requirement of these TEGs, we discuss their material synthesis methods, fabrication techniques and possible mechanisms in detail. For comparison, a few traditional TEMs are also reported with the addition of current and potential advanced general applications of TE materials in the form of rigid and wearable devices.
\end{abstract}

\section{Introduction}

Due to the rapidly depleting conventional energy resources, the present era is on the verge of a severe energy crisis. Additionally, these resources create environmental pollution, leading to the production of greenhouse gases and global warming effects. Accordingly, the rapid technological development and global warming issues have renewed immense interest in the thermoelectric (TE) effect for several applications such as refrigeration, electricity generation, heating, air conditioning, biomedical devices, military devices, aerospace instruments, automobile parts, wearable textiles, and domestic and commercial products. The co-friendly characteristics, easy fabrication and encapsulation, simple mechanism, durability, and portability of thermoelectricity make it suitable for a wide range of industrial applications. ${ }^{1}$ Recently, researchers have mainly been focused on direct solar energy-based power generation techniques such as magnetohydrodynamic, thermoelectric, alkali metal-based thermoelectric,

\footnotetext{
${ }^{a}$ Nano Chemistry and Quantum Dots $R \&$ D Laboratory, Department of Applied Chemistry, Defence Institute of Advanced Technology (DIAT), Ministry of Defence, Govt. of India, Girinagar, Pune-411025, India.

E-mail: pawankhanna2002@yahoo.co.in,morepriyesh@yahoo.com

${ }^{b}$ Mads Clausen Institute, SDU NanoSYD, University of Southern Denmark, Alsion 2, DK-6400, Sønderborg, Denmark
}

piezoelectric and thermo-ionic methods, which can directly convert solar heat or waste heat to electricity. ${ }^{2}$ Direct solar energy-based power generating tools (non-conventional energy devices) are fascinating electricity generation technologies because they do not include any intermediate mechanical conversion processes compared with conventional methods. Thermoelectric power generation is ideal for industries such as the defence industry, and deep space exploration, where system stability, long service life and noiseless systems are highly required together with energy distribution in small areas. Furthermore, thermoelectric technologies, which are reliable, silent, and stable in operation, can be easily extended in the above-mentioned sectors. ${ }^{2}$ However, their development is limited due to low efficiencies achieved from the various available materials.

Thermoelectric generators (TEGs) are known as solid-state fuel-free, reliable devices, which can convert waste heat to electricity directly, without involving any mechanical step, ${ }^{3}$ as shown in Fig. 1. Thermoelectric materials facilitate energy conversion between electrical power and heating/cooling based on the principles of the Seebeck effect and the Peltier effect. The Baltic German physicist Thomas Johann Seebeck pioneered the thermoelectric effect in late $1822,{ }^{4}$ while the French physicist Jean Charles Peltier pioneered the Peltier effect later 
in $1834 .^{5}$ According to Seebeck, when a temperature difference is applied between two different electrical conductors or semiconductors in a closed circuit, it will produce a voltage difference between the two substances. Here, the proportionality constant is known as thermopower or Seebeck coefficient: $\alpha=-\Delta V / \Delta T^{2}$. The reverse phenomenon is known as the Peltier

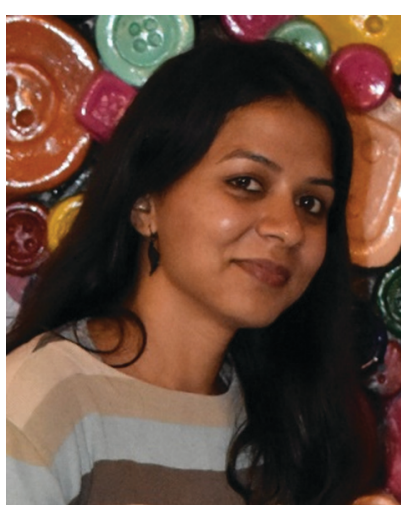

Neha Bisht
Neha Bisht obtained her BTech Degree in Chemical Engineering from BT Kumaon Institute of Technology (BTKIT), Dwarahat, Uttarakhand India in 2014. Continuing her studies, she received MTech Degree in Materials Science and Chemical Technology from the Defence Institute of Advanced Technology, Pune, India in the Department of Applied Chemistry in 2019, where she was a Gold medalist. During her study for her Master's Degree, she received the All India Council Technical Education (AICTE) Fellowship through Graduate Aptitude Test in Engineering (GATE). She is currently working as a Project Assistant under the guidance of Prof. P. K. Khanna at DIAT, Pune, India. She is a life member of the "Society for Materials and Chemistry (SMC)", BARC, Mumbai. She is a lifetime member of "DIAT Alumni Association". Her research interests include nanomaterials, hybrid nanocomposites, thermoelectric materials, metal oxides, ternary metal chalcogenides and their energy applications.

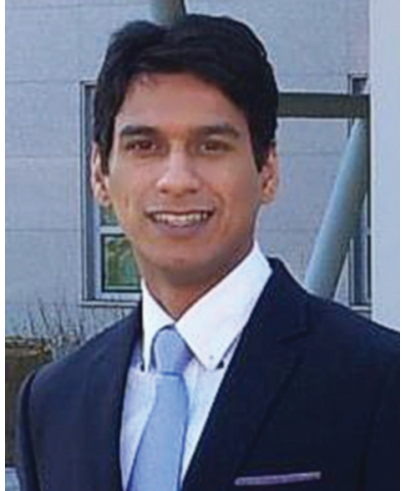

Priyesh More

Dr Priyesh was a faculty member of the Department of Applied Chemistry, Defence Institute of Advanced Technology (DIAT), Pune, India. He is now an entrepreneur and also works as a consultant in the field of nanotechnology. He has a Master's Degree in Polymer Science (2008) from University of Pune. He was awarded a PhD in the major "Nanomaterials Science and Engineering" from the University of Science and Technology, KRICT campus, Daejeon, South Korea. He was awarded with the prestigious "DST INSPIRE Faculty Award" by the Department of Science and Technology, Govt. of India in 2013. His primary research interests are quantum dot technology and customized nanomaterials for advanced applications such as thermoelectrics, sensors, and photocatalysis. He has published 48 research articles in various international journals in the area of nanomaterials.

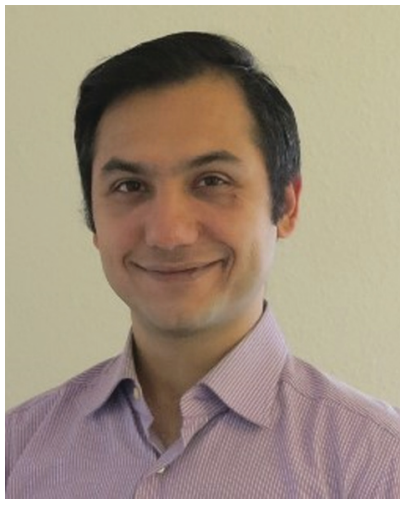

Reza Abolhassani

Reza Abolhassani is a PhD student under the supervision of Professor Yogendra Kumar Mishra at Smart Materials Group, NanoSYD, Mads Clausen Institute, University of Southern Denmark (SDU), Sønderborg, Denmark. He obtained his BSc in Electrical Engineering from K.N.T. University of Technology, Iran, and his MSc in Nanotechnology from University of Southern Denmark. In the Smart Materials Group in NanoSYD, he is developing complex-shaped nanostructures based on $3 D$ nanomaterials for applications in smart technologies.

organometallic chemistry at University of St. Andrews, Scotland (UK) with Professor David J Cole-Hamilton. He is currently a Professor in the Dept. of Applied Chemistry at the Defence Institute of Advanced Technology, Pune, India. His research interests include organometallic chemistry, materials chemistry, nanochemistry of biological importance, quantum dots, nano-inks, nano-fluids and photocatalysis. He has published over 200 research papers. He has recently been named as the top 1\% materials' scientist in the world by a study conducted by Stanford University, USA. He has guided more than 60 masters, doctoral and post-doctoral researchers and completed 20 research projects funded by Govt. Agencies. 


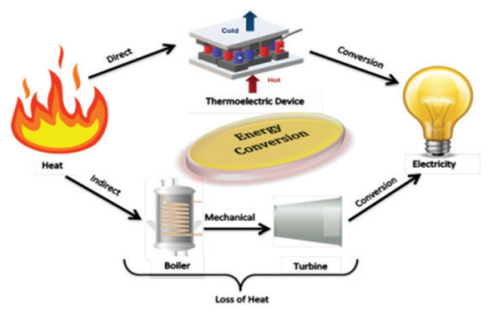

Fig. 1 Schematic representation of electricity generation by comparing the energy conversion between a thermoelectric device and conventional thermal plant.
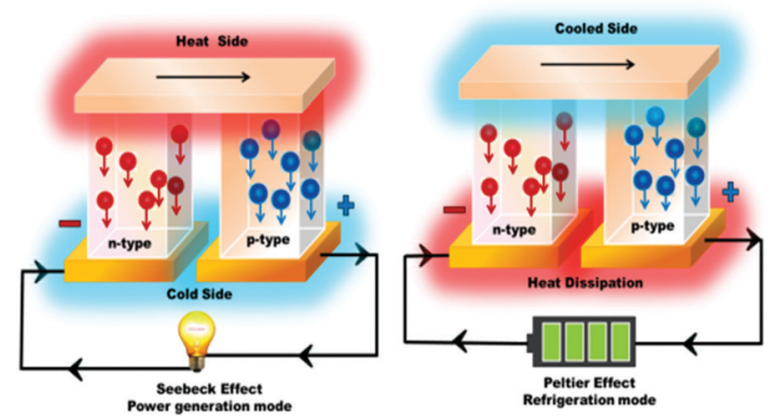

Fig. 2 Schematic of the thermoelectric and Peltier cooling modules.

effect, which is shown in Fig. 2. The performance of thermoelectric materials can be calculated using two parameters, namely the figure of merit (ZT) and power factor (PF). ZT depends on three basic materials properties, including the

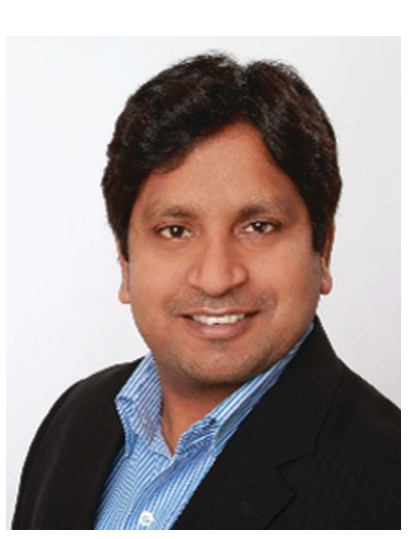

Yogendra Kumar Mishra
Yogendra Kumar Mishra is Professor MSO at Mads Clausen Institute, University of Southern Denmark (SDU), Sønderborg, Denmark. Prior to SDU, he was leading a scientifically independent group at Functional Nanomaterials Chair, Institute for Material Science, Kiel University, Germany. $\quad \mathrm{He}$ obtained Habilitation in Materials Science from Kiel University in 2015 and PhD in Physics in 2008 from Inter University Accelerator Centre/ Jawaharlal Nehru University, New Delhi, India. At SDU Sønderborg, he heads the 'Smart Materials' group with the main focus on the development of sustainable $3 D$ nanomaterials for advanced technologies via green transition. He has published more than 200 research papers. thermopower or Seebeck coefficient $(\alpha)$, electrical conductivity $(\sigma)$, and thermal conductivity $(k)$. Here, the total thermal conductivity is $k=k_{\mathrm{e}}+k_{\mathrm{ph}}$, where $k_{\mathrm{e}}$ and $k_{\mathrm{ph}}$ represent the heat carried by electrons and phonons.

ZT can be defined as: ZT $=\frac{\alpha^{2} \sigma T}{k}$ and the power factor can be calculated by $\mathrm{PF}=\alpha^{2} \sigma$

Additionally, the thermoelectric device efficiency $\left(\eta_{\mathrm{TE}}\right)$ can be represented as

$$
\eta_{\mathrm{TE}}=\eta_{\mathrm{C}}\left(\frac{\sqrt{1+\mathrm{ZT}}-1}{\sqrt{1+\mathrm{ZT}}+T_{\mathrm{H}}-T_{\mathrm{C}}}\right)
$$

where $\eta_{\mathrm{C}}$ denotes the Carnot efficiency, $\eta_{\mathrm{C}}=\left(T_{\mathrm{H}}-T_{\mathrm{C}}\right) / T_{\mathrm{H}}$ and $T_{\mathrm{H}}$ and $T_{\mathrm{C}}$ signify the hot and cold side temperatures, respectively. ${ }^{6}$ A survey of the literature shows that the efficiency and power output of a TEG produced by different methods is quite challenging to achieve.

In the last two decades, researchers have focused on exploring high-performance thermoelectric materials and significantly improving the ZT value by developing new TE materials. Thus, the conventional thermoelectric materials have been grouped according to their structure and composition, including chalcogenides, clathrates, skutterudites, half-Heuslers, silicides, and oxides. Based on their different working temperature range, these TE materials provide higher ZT values and higher efficiency, as shown in Fig. 3..$^{7-10}$ The different bulk nanostructured materials show different ZT values for TEG systems, i.e. PbTe-SrTe (0.77), clathrates (0.85), skutterudites (0.70), HH alloys (0.73), and TE oxides (0.43). ${ }^{11}$ However, from the mid-1990s, major attention was focused on inorganic chalcogenide-based

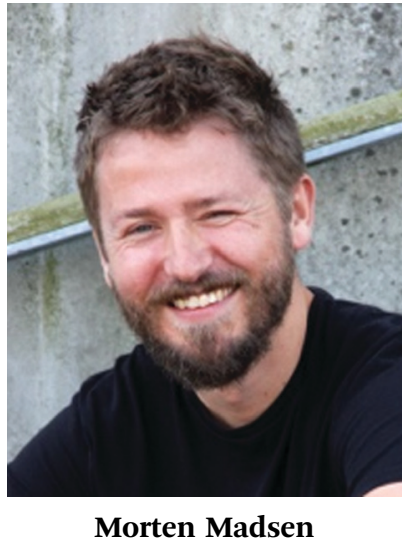

Morten Madsen is a Professor MSO at the University of Southern Denmark (SDU), Head of the SDU OPV Research Group, and Head of the SDU R2R Facility. He obtained his PhD on The Directed Growth of Organic Thin Films and Nanowires (SDU, 2009), and was subsequently a Postdoctoral Fellow in the Javey Research Lab, UC Berkeley, California (2010-2011), working on high performance transistors from III-V nanoscale membranes. He started research in 2011 in the OPV group at SDU NanoSYD, where the focus is on improving the performance of organic and hybrid solar cells, in particular studying the detailed role of interlayers and interfaces on device performance and stability. Since 2016, he has also had a strong focus on device up-scaling through roll-to-roll printing technology at the SDU R2R Facility. He is a coordinator and PI in several international (e.g., ITN Marie Curie, H2020 RIA) and national (e.g., DFF, Villum) research and innovation projects, centered around the topics of organic and hybrid photovoltaics. He is also the supervisor of $10 \mathrm{PhD}$ students and 10 postdocs. 

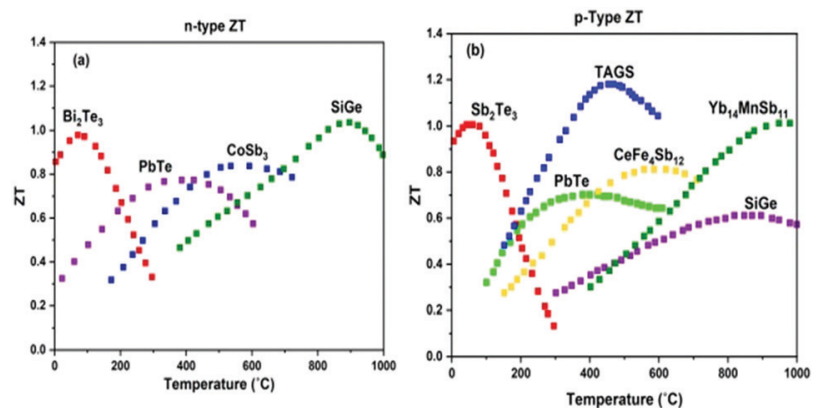

Fig. 3 ZT values of different materials in different temperature ranges (a) for $n$-type and (b) for $p$-type materials. Commercial alloys of bismuth and antimony tellurides from Marlow industries (unpublished data); types of doped $\mathrm{PbTe}^{7}$ skutterudite alloys of $\mathrm{CoSb}_{3}$ and $\mathrm{CeFe}_{4} \mathrm{Sb}_{12}$ from JPL, Caltech (unpublished data); TAGS; ${ }^{8}$ doped SiGe; ${ }^{9} \mathrm{Yb}_{14} \mathrm{MnSb}_{11},{ }^{10}$ Redrawn from ref. 8 .

nanostructured materials because of their higher ZT $(>1)$ and lower thermal conductivity value compared to their bulk inorganic counterparts for widespread use in TE applications, where bismuth telluride ${ }^{12,13}$ and lead telluride ${ }^{14}$ alloys were the most prominent. Lead telluride generally demonstrates superior TE properties at a higher temperature (nearly 500-600 ${ }^{\circ} \mathrm{C}$ ). However, due to its toxic nature, it was replaced by antimonyand selenium-based materials. ${ }^{15}$ Additionally, the scarcity of tellurium in nature led its use as tellurides only for some exceptional applications.

Furthermore, the conventional thermoelectric materials, which are rare, expensive, brittle, and toxic in nature, exhibit processability issues. ${ }^{16}$ Thus, to date, for practical applications, the achieved higher efficiencies of these materials are still lagging behind the maximum possible Carnot efficiency. ${ }^{17}$ Besides, thermoelectric generators require an assembly of dozens or even hundreds of thermoelectric modules to achieve higher performance. Thus, a large gap exists between the efficacy of TE materials and their modules for advanced applications. ${ }^{18}$ Hence, for the efficient use of TE materials in practical applications for energy conversion, we may have to consider: (a) the stability of TE materials under a large temperature gradient, (b) change in their mechanical properties under thermal cycling, and (c) large interfacial contact resistances such as thermal and electrical. ${ }^{11}$

Recently, due to technological challenges, researchers started developing advanced materials with enhanced thermoelectric properties, e.g. binary hybrid nanocomposites of organicconducting polymer and ternary hybrid nanocomposites comprised of organic-inorganic nanoparticles-conducting polymer to augment the thermoelectric performance. In general, organic materials such as graphene (GR), carbon nanotubes (CNT), multi-walled carbon nanotubes (MWCNTs), and reduced graphene oxide (RGO) possess higher thermal conductivities and poor thermoelectric conversion efficiency in comparison to their inorganic counterparts. ${ }^{19}$ However, carbon-based organic materials are abundant, non-toxic and show superior mechanical properties such as low mass density $\left(\sim 1 \mathrm{~g} \mathrm{~cm}^{-3}\right)$, which is almost seven times less than that of bulk inorganic $\mathrm{Bi}_{2} \mathrm{Te}_{3}\left(7.86 \mathrm{~g} \mathrm{~cm}^{-3}\right) .{ }^{19,20}$
Therefore, the limitations of single-phase thermoelectric materials can be overcome by blending organic-inorganic nano-materials to achieve superior tensile strength and controllable Seebeck coefficients in nanocomposite materials. ${ }^{19,21}$ In addition, conducting polymers such as polyaniline (PANI), polypyrrole (PP), polythiophene (PT), polyacetylene (PA), poly(3,4-ethylenedioxythiophene) (PEDOT), polystyrene sulfonate (PSS), and poly(3-hexylthiophene) (P3HT) possess modest electrical conductivity and lower thermal conductivity. ${ }^{22}$ The maximum reported TE properties of the conducting polymer PEDOT:PSS near room temperature is a power factor of $469 \mu \mathrm{W} \mathrm{mK}^{-2}$ with a $\mathrm{ZT}$ value of $0.42 .{ }^{23}$ Accordingly, impregnating conducting polymers into carbon-based materials can increase the Seebeck coefficient and overall power factor by lowering the thermal conductivity of carbon materials. ${ }^{24}$ However, embedding nanoparticles of inorganic materials alone in a conducting polymer matrix may adversely affect the overall electrical conductivity of the composite due to the poor interconnectivity between the particles compared to their inorganic counterparts. ${ }^{25}$ Furthermore, the sensitive nature of the charge transport properties of conducting polymers to oxygen and inorganic nanostructures to di-oxygen (formation of an oxide layer) has been shown to potentially damage the electrical conductivity of conducting polymers, which limits the use of this type of material for TE application. ${ }^{26}$ Additionally, the solution processability of conducting polymers at a low temperature has a great advantage in the large-scale production of hybrid nanocomposites. Therefore, the use of fascinating printing methods such as aerosol jet printing, ${ }^{24}$ screen printing, ${ }^{27,28}$ drop-casting, ink-jet printing, ${ }^{29,30}$ spin coating, ${ }^{31}$ slot-die coating, ${ }^{32}$ spray coating, ${ }^{33}$ and dispenser printing $^{34}$ makes it possible to fabricate fully flexible $\mathrm{TE}$ devices. For example, polyacetylene exhibits the highest thermoelectric properties with the highest power factor of $10^{-4} \mathrm{~W} \mathrm{~m}^{-1} \mathrm{~K}^{-2}$ due to its excellent electrical conductivity among the conducting polymers, but its ageing and environmental instability exclude it from the TE list. ${ }^{35}$ In contrast, other conducting polymers such as PEDOT:PSS and PANI are prospective candidates for TE applications. PANI exhibits a large electrical conductivity of $10^{5} \mathrm{~S} \mathrm{~m}^{-1} \cdot{ }^{36}$ Furthermore, conducting polymers are used widely because of their easy processing $^{32}$ and environmental stability. ${ }^{37}$ However, the reduced electrical conductivity and lower power factor in the order of $10^{-5}$ to $10^{-8} \mathrm{~W} \mathrm{~m}^{-1} \mathrm{~K}^{-2}$ limit the use of conducting polymers as single-phase materials in TE applications. ${ }^{26}$ Furthermore, hybrid nanocomposites furnish lightweight, flexible, low cost, easily processable and scalable TE materials. Therefore, the attractive features of organic-inorganic-conducting polymer composites such as superior mechanical strength, good thermoelectric properties, and solution processability are favorable for highly efficient wearable thermoelectric generators.

Generally, there are three types of thermoelectric generators (TEGs) as follows: (1) bulk TEGs are useful for high power application such as in automobiles, power plants, aerospace, and various factories. (2) Thin-film TEGs, which may be handy for low power applications such as electronic and biomedical devices. Thin-film TEGs require a thickness of 
fewer than 50 micrometres, and they are smaller in size than bulk TEGs. $^{38,39}$ (3) Smart and intelligent textile TEGs, which work based on their functionality in astronaut suits, biomedical devices, military and other intelligent devices, where a portable power source is preferred. Despite various efforts to insert TE materials into textiles for harvesting energy from body heat, the power level of these arrangements is quite low for practical wearable applications. In addition, upon various deformation resistances such as stretching, bending, twisting, folding and pressing, wearable TE devices should maintain a steady performance. ${ }^{40}$ However, approaches for bulk TEGs in comparison to thin-film-, printed- and textile-based TEGs demonstrate significantly higher power conversion due to the maintenance of an appropriate temperature difference across the TE junction. ${ }^{41}$

To obtain higher performances from wearable TE devices, they must be optimized by TE material selection, preparation method, device fabrication, choice of filler materials, ${ }^{42,43}$ geometry of legs, ${ }^{44-46}$ heat spreader effect, ${ }^{44,47}$ type of metal electrode and substrate, ${ }^{48-51}$ and matching of thermal load. ${ }^{52-54}$ The major requirements for an embedded TEG in wearable textiles include greater ZT $(>1)$, higher thermopower and lower thermal conductivity at room temperature. The low thermal conductivity of the material will maintain a higher temperature gradient across hot and cold surfaces and a higher Seebeck coefficient will provide sufficient voltage for the smooth operation of the subsequent DC-DC boost convertor. Another challenge for thermoelectrics is the low ZT of n-type materials, which can be improved by increasing the large number of valleys near the conduction band edge. These factors can be addressed by developing a new synthetic methods. The generated power output per unit area does not depend on the number of thermoelectric legs and the cross-sectional area of the legs, it only associated with the ratio of the insulated cross-sectional areas and the length of the thermoelectric legs. ${ }^{55}$ Therefore, finding very low thermally conducting filler materials can minimize the lateral heat loss through space and can improve the power output. The fill factor is the ratio of space occupied by the TE material to the total surface area occupied by the module. ${ }^{56}$ The geometry of the leg greatly influences the TEG performance. Thus, increasing the leg length in the aspect ratio, changing the leg shape, and reducing the fill factor can elevate $\Delta T .{ }^{57}$ Heat spreaders possessing high thermal conductivity can be placed on both sides of wearable TEGs to enhance their overall power output. These are preferable instead of finbased heat sinks because of their flexibility and light weight. ${ }^{41}$ Selecting a metal interconnected with high electrical conductivity and matching thermal expansion coefficient with TE legs, for wearable TEGs is an important parameter. ${ }^{48}$ The device performance can be improved by minimizing the interfacial contact resistance between the legs and interconnects. Wearable TEGs require a flexible substrate, which should be compatible with the human body and possess a low thermal resistance for improved performances, e.g. polydimethylsiloxane (PDMS) and Kapton. ${ }^{58}$ Another necessary factor for improving device performance is the thermal resistance of TEGs, which must be equal and comparable to the parasitic thermal resistances. The thermal resistance of TEGs can be enhanced by two ways, lowering the fill factor or reducing the thermal conductivity of the leg material. ${ }^{44}$

Furthermore, despite the compact size, easy operation, longer lifetime, and low maintenance $\operatorname{cost}^{2}$ of TE devices, they are still lagging behind other conventional energy generation technologies. Specifically, in the current market, the thermoelectric device efficiency is limited to $5-6 \% .^{59,60}$ Therefore, this type of energy transformative technology requires four vital conditions for bringing it into practice as follows: (1) simple processes and scalable materials to match the energy usage in terms of economic and physical magnitude, (2) the capability of economical maintenance during manufacturing and recycling, (3) compatibility and integrability with the existing manufacturing infrastructure, and (4) improved performance for a specific niche application. With the development and advancement of nanotechnology, these energy devices can become more practically viable. Thus, the above-mentioned four conditions establish a strong foundation for future research in flexible and rigid nanostructured thermoelectric generators. ${ }^{61}$ Presently, the thermoelectric technology market is growing and demanding because it is capable of converting any waste heat and solar radiation to generate more power. To extract the maximum power from TEGs and boost their efficiency, relevant investigations in many subareas are being carried out by researchers to optimize TE devices. ${ }^{2,59}$

This review comprehensively covers the progress of hybrid nanocomposites towards a variety of applications including flexible/wearable devices. The introduction covers the general background of thermoelectrics, their working principle, advancement, and requirement of TE materials. To bridge the knowledge gap between advanced and conventional TE materials, a few traditional TE materials are also discussed. The next section highlights the well-developed hybrid nanocomposites in detail by focusing on their manufacturing techniques, materials used, device fabrication, device performance, and working mechanism. In addition, the use of traditional to hybrid $\mathrm{TE}$ materials in primary industrial applications is addressed briefly. The application section covers the use of both rigid and flexible/wearable thermoelectrics in various sectors. The final section presents our conclusions by providing various solutions to improve the TE efficiency.

\section{Conventional TE materials}

Recently, various conventional TE materials and their devices have been reported by researchers. Different materials have been utilized in the fabrication of TEGs for significantly improving their performance. The different characteristics of these materials make them appropriate for use in device manufacturing techniques. Accordingly, this section describes the various traditional thermoelectric materials and their selection criteria based on their properties and working temperature range for use in thermoelectric applications. ${ }^{62}$ 


\subsection{BiTe alloys}

Bismuth tellurides and their alloys are widely established and efficient materials in the field of thermoelectric generators since the 1950s. The p-type $\mathrm{Bi}_{x} \mathrm{Sb}_{2-x} \mathrm{Te}_{3}$ and n-type $\mathrm{Bi}_{2} \mathrm{Te}_{1-x} \mathrm{Se}_{x}{ }^{7}$ are already being used in commercial TE cooling at RT and power generation modules at a temperature not exceeding $500 \mathrm{~K} .{ }^{63}$ Group-IV tellurides generally function in the temperature range of $600-800 \mathrm{~K}^{64}$ In 1952 , Ioffe reported the interesting behaviour of semiconductors according to their position in the periodic table. He discovered that elements possessing a large atomic weight show lower thermal conductivity. ${ }^{65}$ Thus, based on this fact, $\mathrm{Bi}_{2} \mathrm{Te}_{3}$ and its alloys were selected as potential thermoelectric materials because of their heavy weight, narrow bandgap, and large charge mobility, which impart high phonon scattering due to smaller phonon group velocity and lower thermal conductivity compared to other heavy-weight semiconductors. ${ }^{66}$ However, baseline alloys of the bulk material show the highest ZT value of 1 , which is inefficient. Therefore, to improve the ZT value, various nanocomposites of these alloys have been reported. Moreover, embedding nanoparticles in the bulk matrix to prepare nanocomposites, which is known as 'nano-inclusion', can reduce the lattice thermal conductivity of nanocomposites without damaging the thermoelectric performance of materials. ${ }^{67}$ Based on this phenomenon, Fan et al. ${ }^{67}$ synthesized nanocomposites of p-type $\mathrm{Bi}_{0.4} \mathrm{Sb}_{1.6} \mathrm{Te}_{3}$, in which $40 \%$ of their nanostructured particles were less than $200 \mathrm{~nm}$ in size and $60 \%$ were micronsized, using a spark plasma-sintering method to obtain a ZT value of 1.8 at $43{ }^{\circ} \mathrm{C}$. Other p-type materials showed a ZT of 1.56 for $\mathrm{Bi}_{0.52} \mathrm{Sb}_{1.48} \mathrm{Te}_{3}$ at $27{ }^{\circ} \mathrm{C}, 1.50$ for $\mathrm{Bi}_{0.48} \mathrm{Sb}_{1.52} \mathrm{Te}_{3}$ at $117{ }^{\circ} \mathrm{C}$ and 1.50 for $\left(0.3\right.$ vol\% $\left.\mathrm{Al}_{2} \mathrm{O}_{3}\right) / \mathrm{Bi}_{0.5} \mathrm{Sb}_{1.5} \mathrm{Te}_{3}$ at $50{ }^{\circ} \mathrm{C} .{ }^{68-70}$ Yan et al. ${ }^{71}$ reported the synthesis of an n-type $\mathrm{Bi}_{2} \mathrm{Te}_{2.7} \mathrm{Se}_{0.3}$ nanocomposite (NC) using ball milling and hot pressing to obtain a high ZT value 1.04 at $125{ }^{\circ} \mathrm{C}$. The obtained mean particle size of the nanocomposite was 1-2 $\mu \mathrm{m}$. The nanocomposites synthesized by the spark plasma-sintering (SPS) method were better than highenergy ball milling (HEBM) because SPS results in the formation of coherent grain boundaries, whereas the HEBM method results in random grain boundaries. ${ }^{68}$ Cao et al. ${ }^{72}$ employed a hydrothermal method to prepare p-type (Bi Sb) ${ }_{2} \mathrm{Te}_{3}$, where the ZT of 1.47 at $167{ }^{\circ} \mathrm{C}$ was obtained. Some researchers have reported flexible wearable TEGs using BiTe alloy nanocomposite ink or paste using different methods and substrates. Cao et al. ${ }^{73}$ fabricated a screen-printed flexible thick film thermoelectric generator on a polyimide substrate from bismuth and antimony tellurium with a low material resistivity. The fabricated prototype resulted in an output voltage and power of $36.4 \mathrm{mV}$ and $40.3 \mathrm{nW}$ at $\Delta T$ of $20{ }^{\circ} \mathrm{C}$. Francioso et al. ${ }^{58}$ reported a wearable micro TEG, generating an output voltage and power of $430 \mathrm{mV}$ and $32 \mathrm{nW}$, respectively, using 100 thin-film pairs of p-type antimony tellurides and n-type bismuth tellurides at a $\Delta T$ of $40{ }^{\circ} \mathrm{C}$. Kim et al. ${ }^{74}$ reported the fabrication of a wearable-type TEG on a polymerbased fabric via a dispenser printing method. The TE device consisted of 12 pairs of $\mathrm{Bi}_{0.5} \mathrm{Sb}_{1.5} \mathrm{Te}_{3}$ and $\mathrm{Bi}_{2} \mathrm{Se}_{0.3} \mathrm{Te}_{2.7}$ over an area of $6 \times 25 \mathrm{~mm}^{2}$, connected by a conductive thread, which produced $224 \mathrm{nW}$ power at a $\Delta T$ of $15 \mathrm{~K}$. Madan et al. ${ }^{75}$ reported the synthesis of n-type bismuth tellurides powders by mixing $\mathrm{Bi}$ and Te in a molar ratio of $36: 64$ and $2 \mathrm{wt} \%$ using selenium as a filler by mechanical blending. Epoxy resins were added to the material in a 20:80 ratio as a binder. The obtained ZT was 0.17 at $300 \mathrm{~K}$. A TEG $(5 \mathrm{~mm} \times 400 \mu \mathrm{m} \times 120 \mu \mathrm{m})$ was fabricated, containing 20 single legs of n-type material on a polyimide substrate using an evaporated gold contact as the electrode, which generated an output power, voltage, and current of $1.6 \mu \mathrm{W}, 40 \mathrm{mV}$ and $40 \mu \mathrm{A}$ at a $\Delta T$ of $20^{\circ} \mathrm{C}$, respectively. However, the insulating effect of the epoxy resin resulted in poor thermal conductivity and electrical conductivity, which affected the overall ZT value. Nishino et al. $^{76}$ fabricated two types of flexible TEGs using 54 thermocouples (TCs) $/ \mathrm{cm}^{2}$ of $\mathrm{Bi}_{2} \mathrm{Te}_{3}$-and nickel alloy in a $25 \mathrm{~cm}^{2}$ polyimide substrate. The $\mathrm{Bi}_{2} \mathrm{Te}_{3}$-based TEG consisted of $\mathrm{Bi}_{0.3} \mathrm{Sb}_{0.7} \mathrm{Te}_{3}$ as the p-type and $\mathrm{Bi}_{2} \mathrm{Te}_{3}$ as the n-type material. In contrast, the nickel-based alloy consisted of $\mathrm{Ni}_{0.9} \mathrm{Cr}_{0.1}$ alloy as the p-type and $\mathrm{Ni}_{0.52} \mathrm{Cu}_{0.48}$ alloy as the n-type material. The produced power output by the $\mathrm{Bi}_{2} \mathrm{Te}_{3}$-based and nickel alloybased TEG was $100 \mu \mathrm{W}$ and $75 \mu \mathrm{W}$ at $\Delta T$ of $10 \mathrm{~K}$, respectively. Shin et $a l^{77}$ reported a flexible TEG on a fiberglass fabric using inks of $\mathrm{p}$-type $\mathrm{Bi}_{0.5} \mathrm{Sb}_{1.5} \mathrm{Te}_{3}$ and n-type $\mathrm{Bi}_{2} \mathrm{Te}_{2.7} \mathrm{Se}_{0.3}$. Organic solvents and methylcellulose as a binder were used in a very small concentration of $0.45-0.60 \mathrm{wt} \%$ for the formation of the ink. The obtained ZT for the p-type and n-type materials was 0.65 and 0.81 at RT, respectively. Suarez et al. ${ }^{78}$ reported a transformed rigid TEG to an EGaIn-based flexible TEG. Bulk thermoelectric legs of $\mathrm{Bi}_{2} \mathrm{Te}_{3}$ and $\mathrm{Sb}_{2} \mathrm{Te}_{3}$ were used as the TE legs, which were connected by liquid gallium-indium on a PDMS substrate. The obtained ZT value, voltage and power output were

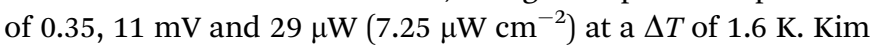
et $a l^{28}$ reported a wearable flexible TEG by screen-printing technology to print thick films on a glass fabric using bismuth and antimony telluride paste. The power density exhibited by the TEG was $3.8 \mathrm{~mW} \mathrm{~cm} \mathrm{~cm}^{-2}$ and $28 \mathrm{~mW} \mathrm{~g}^{-1}$ at a temperature difference of $50 \mathrm{~K}$. The device allowed a minimum bending radius of $20 \mathrm{~mm}$, and no major change was noticed in performance after 120 cycles of repeated bending. Considering that different ZT values were obtained from different synthetic methods, one has to compensate with process simplicity, cost effectiveness and scalability of materials. ${ }^{79}$ The above-mentioned reported thermoelectric properties for $\mathrm{Bi} / \mathrm{Te} / \mathrm{Sb}$ materials are presented in Table 1.

\subsection{PbTe and PbSe alloys}

PbTe and its alloys are heavy materials, similar to BiTe alloys. ${ }^{80,81}$ The p-type $\mathrm{PbTe}_{1-x} \mathrm{Se}_{x}$ and n-type $\mathrm{Pb}_{1-x} \mathrm{Sn}_{x}$ Te are types of PbTe alloys that usually show ZT values of close 1 at $300{ }^{\circ} \mathrm{C},{ }^{82,83}$ whereas their bulk alloys show a ZT value of close to 0.7 at $467{ }^{\circ} \mathrm{C} .{ }^{80,81}$ Recently, efforts have been focused on obtaining PbTe alloys with a ZT value in the range of 1.20-2.20 together with band engineering to optimize the performance of TEGs by reducing their thermal conductivity. ${ }^{63,79,84}$ The melt growth method was used by Hsu et al. ${ }^{85-87}$ to synthesize n-type $\mathrm{AgPb}_{18} \mathrm{SbTe}_{20}$, obtaining a higher ZT of $\sim 2.2$ at $527{ }^{\circ} \mathrm{C}$. The thermal conductivity of the nanocomposite was reduced by placing precipitates of $\mathrm{Ag}$ nanoparticles into the crystal matrix. In addition, similar results were obtained for n-type 
Table 1 Thermoelectric properties of $\mathrm{Bi} / \mathrm{Sb} / \mathrm{Te}$-based thermoelectric materials

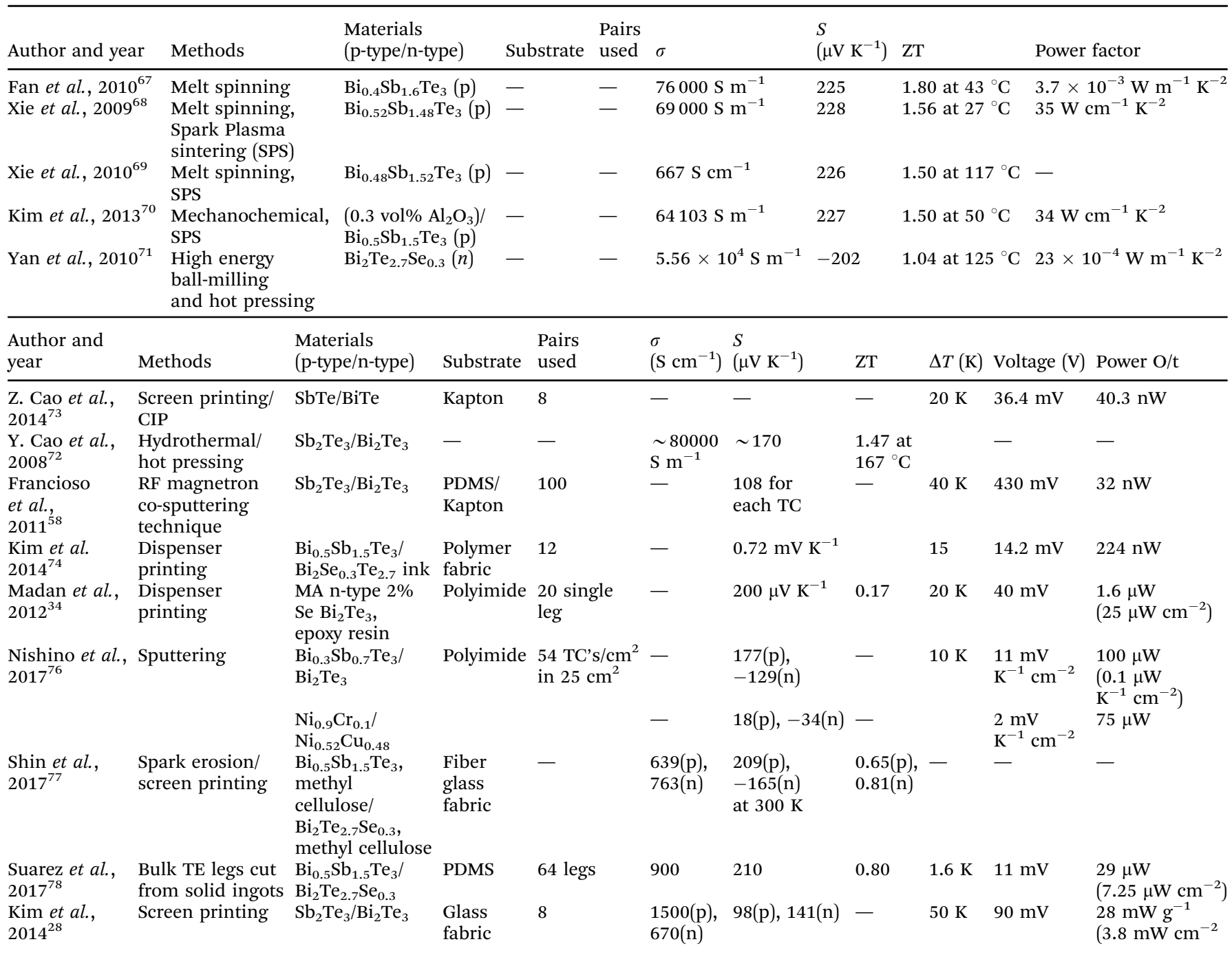

$\left(\mathrm{Pb}_{0.95} \mathrm{Sn}_{0.05} \mathrm{Te}\right)_{0.92}(\mathrm{PbS})_{0.08}$ and $\mathrm{Ag}_{0.5} \mathrm{~Pb}_{18} \mathrm{Sb}_{1.2} \mathrm{Te}_{20}$ as well as p-type $\mathrm{Ag}_{0.5} \mathrm{~Pb}_{6} \mathrm{Sn}_{2} \mathrm{Sb}_{0.2} \mathrm{Te}_{10}, \mathrm{Na}_{0.95} \mathrm{~Pb}_{20} \mathrm{SbTe}_{22}$ and $2 \%$ sodium doped PbTe-PbS. ${ }^{88-91}$ According to Biswas et al. ${ }^{84}$ an increased ZT value of 2.2 at $642{ }^{\circ} \mathrm{C}$ was obtained for p-type Na-doped $\mathrm{PbTe}-\mathrm{SrTe}$, in which endotaxial SrTe nanocrystals having a grain size of 2-10 $\mathrm{nm}$ were embedded in the sodium-loaded PbTe matrix. Further, lead selenides can be investigated as a substitute for lead tellurides because tellurium is rarely found in the Earth's crust $(<0.001 \mathrm{ppm})$, while the abundance of selenium is equal to $0.5 \mathrm{ppm}^{16}$ In addition, PbSe (n-type) doped with aluminium showed a ZT of 1.3 and PbSe (p-type) doped with sodium showed a ZT of 1.2 at $577^{\circ} \mathrm{C}$. However, the $\mathrm{ZT}$ values of PbSe alloys were found to be less than that of PbTe alloys. ${ }^{92,93}$ Later, Wang et al. reported that adding a small quantity of $\mathrm{Sr}$ to $\mathrm{PbSe}$ resulted in an enhancement in $\mathrm{ZT}$, where the ultimate ZT achieved was 1.5 at $657{ }^{\circ} \mathrm{C} .{ }^{94}$

\subsection{Skutterudites}

The general molecular formula for skutterudites is $\mathrm{MX}_{3}$, where $\mathrm{M}$ represents $\mathrm{Co}, \mathrm{Rh}$ or $\mathrm{Ir}$, and $\mathrm{X}$ represents $\mathrm{P}$, As or $\mathrm{Sb}$. Skutterudites as thermoelectric materials have the potential to sustain a high temperature, and thus they can be used in high-temperature operating industries for power generation applications. The complex crystal structures of these materials are responsible for their low thermal conductivity values. ${ }^{95}$ Due to the existence of larger voids within the crystal lattice, the intrinsic sites can be doped with foreign ions, which form filled skutterudites $\left(\mathrm{T}_{y} \mathrm{M}_{4} \mathrm{X}_{12}\right)^{96,97}$ and increase the scattering of lowfrequency phonon. This phenomenon is known as the "rattling effect", ${ }^{95,96}$ which is responsible for improved thermoelectric properties. $\mathrm{CoSb}_{3}$-based skutterudite elements exist in great abundance in the Earth, and thus these materials are becoming more interesting to researches. Furthermore, the versatility of these materials towards accepting various alkalis, alkaline earth metals, lanthanides, actinides and group IV elements as void filler makes them attractive. ${ }^{97,98} \mathrm{Li}$ et al. ${ }^{99}$ reported an n-type $\mathrm{Yb}_{0.2} \mathrm{Co}_{4} \mathrm{Sb}_{12.3}$-filled skutterudite, showing a $\mathrm{ZT}$ value of 1.30 at $527{ }^{\circ} \mathrm{C}$. Nanostructured filling in skutterudites increases the ZT of the system. This was proven by Zhao et al. ${ }^{100}$ when they synthesized n-type $\mathrm{Ba}_{0.14} \mathrm{In}_{0.23} \mathrm{Co}_{4} \mathrm{Sb}_{11.84}$, showing a $\mathrm{ZT}$ value of 1.34 at $577{ }^{\circ} \mathrm{C}$. Shi et al. ${ }^{101}$ reported n-type $\mathrm{Ba}_{0.08} \mathrm{La}_{0.05} \mathrm{Yb}_{0.04} \mathrm{Co}_{4} \mathrm{Sb}_{12}$, resulting in the $\mathrm{ZT}$ value of 1.70 at 
$577{ }^{\circ} \mathrm{C}$, using a high-energy ball milling and nanostructured spark plasma-sintering method. Rogl et al. ${ }^{102}$ reported p-type $\mathrm{Sr}_{0.12} \mathrm{Ba}_{0.18} \mathrm{DD}_{0.39} \mathrm{Fe}_{3} \mathrm{CoSb}_{12}$ with a ZT of 1.30 at $527{ }^{\circ} \mathrm{C}$. The ZT value of p-type skutterudites cannot be improved beyond a limit in comparison to n-type skutterudites because more filling in p-type materials tends to strongly force them to become n-type materials. ${ }^{64}$

\subsection{Half-heuslers}

Half-Heusler compounds show a composition of $\mathrm{X}_{2} \mathrm{YZ}$, where $\mathrm{X}$ and $\mathrm{Y}$ represent the transition metal positions and $\mathrm{Z}$ represents the p-block element. Half-Heusler ( $\mathrm{HH})$ compounds are another group of promising TE materials having a face-centred cubic crystal structure with excellent thermal stability at higher temperatures. Additionally, they are intermetallic compounds with a higher thermopower and relatively higher thermal conductivity compared to other TE materials. ${ }^{95,103}$ However, the development of bulk $\mathrm{HH}$ compounds for thermoelectric application is challenging due to their high thermal conductivity. Therefore, improvement in crystal structure was imparted by nanodimensions, which showed better thermoelectric properties than bulk $\mathrm{HH}$ compounds because nanostructures reduce the lattice thermal conductivity of compounds. This effect can be linked to the work of Joshi et al., ${ }^{104}$ where an n-type $\mathrm{Hf}_{0.5} \mathrm{Zr}_{0.25} \mathrm{Ti}_{0.25}$ $\mathrm{NiSn}_{0.99} \mathrm{Sb}_{0.01}$ compound was synthesized using ball milling and hot pressing, resulting in a ZT of 1 , at $500{ }^{\circ} \mathrm{C}$. Further, Yan et al. ${ }^{105}$ prepared a p-type $\mathrm{Hf}_{0.8} \mathrm{Ti}_{0.2} \mathrm{CoSb}_{0.8} \mathrm{Sn}_{0.2}$ compound by applying an arc melting method followed by high-energy ball milling and hot pressing, resulting in a ZT of 1 at $800{ }^{\circ} \mathrm{C}$.

\subsection{SiGe alloys}

SiGe alloys are another group of promising candidates for hightemperature applications. These alloys exhibit the highest $\mathrm{ZT}$ value at temperatures greater than $900{ }^{\circ} \mathrm{C}$ and possess low-temperature deterioration up to $1000{ }^{\circ} \mathrm{C}$. Bulk $\mathrm{Si}_{0.8} \mathrm{Ge}_{0.2}$ shows a ZT value of nearly 1 and 0.6 for n-type and p-type, respectively. ${ }^{9}$ Wang et al. ${ }^{106}$ reported a high-energy ball milling and hot pressing method for the fabrication of nanostructured SiGe alloys, achieving a peak ZT value of up to 1.3 for n-type at $900{ }^{\circ} \mathrm{C}$ and 1 for p-type at $900-950{ }^{\circ} \mathrm{C}$, whereas their bulk counterpart showed a ZT of 1 at $900{ }^{\circ} \mathrm{C}$ and 0.6 at $900-950{ }^{\circ} \mathrm{C} .{ }^{107}$ Considering that $\mathrm{SiGe}$ is the most expensive thermoelectric material, is widely employed for power generation in space applications, where solar cells are not common. ${ }^{108}$ Furthermore, to improve the thermoelectric material performance, the inclusion of nanostructured materials is necessary. The nanostructured defect reduces the thermal conductivity by imparting more phonon scattering at the junctions. The anharmonic large vibration of guest ions within the oversized atomic cages is known as rattling motion. ${ }^{109}$ Due to the rattling effect in some nanocomposites, nanoparticles may precipitate within the crystal matrices, leading to the scattering of acoustic phonons, which helps in reducing the lattice thermal conductivity without altering the power factor value. The rattling effect has been also observed in thermoelectric materials that possess a complex crystal structure. Besides, the power factor can be enhanced by enforcing a resonant electronic state in the valence band. If the development of nanocomposite materials becomes easy and scalable, they can be used in industrial applications as economic and efficient conversion technology. ${ }^{64,79}$

\section{Hybrid nanocomposite-based TE materials}

Recently, researchers have been focused on improving the efficacy of TE materials by doping them with suitable p-type or n-type dopants, and synthesizing hybrids by combining organic and inorganic materials. Historically, after the discovery of $\mathrm{Bi}_{2} \mathrm{Te}_{3}$ alloys as a TE material in the $1960 \mathrm{~s}$, various efforts have been made by researchers to marginally revamp the TE efficiency of semimetals, which is a challenging task. As discussed above, the conventional thermoelectric semiconducting materials are expensive, difficult to process, and suffering from low reliability, which hinder their widespread use in TEGs for commercial applications. The simple leg-type thermoelectric module structure without any moving parts has a great advantage over conventional energy generation sources (e.g., compressors, turbines and engines). Generally, the high energy densities of TEGs are based on per unit volume and weight, which is perfect for mobile distribution systems. Hybrid nanocomposites and doped organic/inorganic materials are very fascinating as they possess a lower thermal conductivity, higher Seebeck coefficient, higher electrical conductivity, simple processing and manufacturing, and light weight compared to conventional thermoelectric materials. ${ }^{110}$ Herein, we compiled various hybrid nanocomposites reported for their application in wearable TEGs.

\subsection{Doped CNT/graphene-based TE materials}

CNTs and graphene are universally known for their unique physical and chemical properties. A roadmap has been aimed at graphene to make it a potential candidate for flexible $\mathrm{TE}$ devices. Compared to conventional inorganic bismuth tellurides, graphene presents good electrical properties at RT, but poor Seebeck coefficient due to its high thermal conductivity. Thus, to overcome this issue, some researchers initiated doping graphene by various dopants to reduce its thermal conductivity by imparting defects on the CNT/graphene webs. Particularly, most research has been mainly focused on p-type organic TE materials compared to their n-type counterparts. However, n-type TE materials can be achieved by doping CNT webs with appropriate n-type dopants to increase the carrier concentration. Thus far, a charge transfer doping process ${ }^{111,112}$ was developed to prepare electron-rich n-type CNT materials with outstanding TE efficiency. This method is different from the conventional doping methods (substitution) as it does not impart any defects in the $\mathrm{sp}^{2}$-hybridized carbon of the CNT. Most of the reported n-type CNT-based TE materials were developed by the direct doping method. For example, Yu et al. ${ }^{113}$ reported a combined doping method to prepare n-type CNT using polyethyleneimine (PEI) and sodium borohydride to achieve a Seebeck coefficient of $80 \mathrm{mV} \mathrm{K}{ }^{-1}$. Similarly, Nonoguchi et al. ${ }^{114}$ efficiently modulated the n-type single-walled CNT electron 


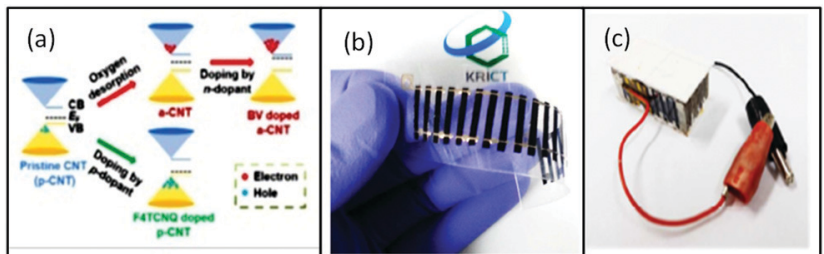

Fig. 4 (a) Schematic of changes in the CNT structure by oxygen desorption and molecular doping, (b) image of a fabricated flexible TE module, and (c) image of a vertical TE device containing 20 layers of 200 $\mathrm{p}-\mathrm{n}$ pairs. Reproduced from ref. 116 with permission from RSC Publications, Copyright 2017

concentration by doping with phosphine containing aromatics. However, all these reports on the direct doping method could not achieve the optimal TE performance because the presence of ambient oxygen adversely affects n-type doping by occupying the n-dopant sites of CNT. ${ }^{115}$ In contrast, An et al. ${ }^{116}$ reported a-CNT webs doped with n-type and p-type molecular dopants in conjunction with annealing to prepare organic semiconductors. The n-type TE material was prepared (Fig. 4) by treating annealed CNT (desorption of oxygen from p-CNT) webs with benzyl viologen (BV), resulting in the highest $\mathrm{PF}$ of $3103 \mu \mathrm{W} \mathrm{mK} \mathrm{mK}^{-2}$. The obtained n-type TE value was superior to that of the non-annealed BV-doped pristine CNT web (p-type) having a power factor of $1901 \mu \mathrm{W} \mathrm{mK}{ }^{-2}$. Therefore, their achieved thermoelectric properties for n-type CNT webs were electrical conductivity of $2228 \mathrm{~S} \mathrm{~cm}^{-1}$, thermopower of $-116 \mu \mathrm{V} \mathrm{K}^{-1}$, thermal conductivity of $5 \mathrm{~W} \mathrm{~m} \mathrm{~m}^{-1} \mathrm{~K}^{-1}$ and ZT of 0.19 at $300 \mathrm{~K}$. Similarly, they prepared a p-type TE by doping the p-CNT web with $\mathrm{F}_{4}$ TCNQ with a concentration of $10 \mathrm{mM}$, which helped in controlling the large electrical affinity. The maximum power factor obtained for the p-type TE was $2252 \mu \mathrm{W} \mathrm{mK}{ }^{-2}$, with the electrical conductivity of $3000 \mathrm{~S} \mathrm{~cm}^{-1}$, Seebeck coefficient of $50 \mu \mathrm{V} \mathrm{K}^{-1}$, thermal conductivity of $6 \mathrm{~W} \mathrm{~m}^{-1} \mathrm{~K}^{-1}$ and ZT of 0.11 . The authors claimed that the synthesized materials showed an enhanced power factor compared to previously reported organic TE materials and the results were identical to the conventional inorganic semiconducting $\left(\mathrm{Bi}_{2} \mathrm{Te}_{3}\right)$ materials at room temperature. Additionally, to fabricate a TE module from p-type and n-type materials, they used a dispensing method. The free-standing CNT webs were adhered on a polyethyleneterephthalate (PET) flexible substrate by Ag paste to make Y-shape (cross) electrical contacts between the $n$ and $\mathrm{p}$ legs. The dimensions of the n-type or p-type effective legs were width of $1.5 \mathrm{~mm}$, length of $12 \mathrm{~mm}$, and thickness of $20 \mu \mathrm{m}$ for a single TE module. Specifically, 10 couples of $\mathrm{p}-\mathrm{n}$ junctions were attached electrically in series and thermally in parallel and sandwiched in the middle of two ceramics plates to deliver homogeneous heat to the TEG. The highest power output of the thermoelectric module was $123 \mu \mathrm{W}$ at $\Delta T=20 \mathrm{~K}$ and the corresponding power density with a planer module structure was $1180 \mu \mathrm{W} \mathrm{cm}{ }^{-2}$. The mechanical flexibility of the TE web was checked by subjecting it to the 25 repeatable folding cycles at $180^{\circ}$ and 25 cycles at $-180^{\circ}$ (total 50 cycles), in which only a small deflection of $1.65 \%$ was observed in internal resistances. Kim et al. ${ }^{117}$ fabricated both $\mathrm{p}$ - and n-type flexible, lightweight fabric-like materials based on CNT. They prepared p-type CNT films by

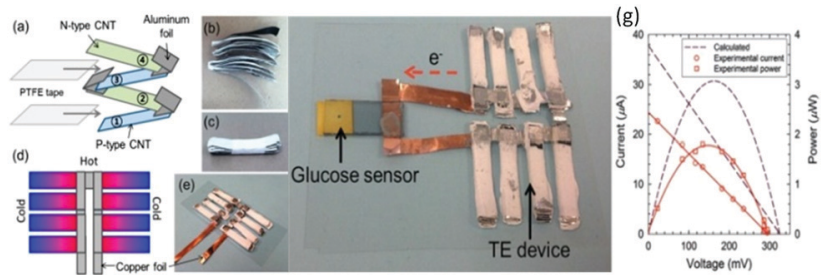

Fig. 5 (a) Assembly process of $p$ - and n-type carbon nanotube films, (b) stack of 9 pairs of $p-n$ type film modules, (c) picture of PTFE-bound module, (d) designed TE device for a given temperature gradient, (e) fabricated thermoelectric device using 144 films of $p$ - and $n$-type material, ( $f$ ) prototype of a glucose detection sensor integrated with a thermoelectric device, and (g) graph of current-voltage and output power with varying load conditions. Reprinted with permission from ref. 117 (S. L. Kim, K. Choi, A. Tazebay, C. Yu, ACS Nano. 8 (2014) 2377-2386). Copyright (2014) ACS Publications.

adding SDBS to a CNT solution followed by vacuum filtration on a PTFE membrane. Similarly, n-type films were prepared using the same procedure except the dopants used were PEI and DETA in a specific ratio. According to the authors, another desirable feature for improving the practical TE efficiency of CNTs is by creating many junctions in their films and designing proper junctions between CNTs and the surface of CNTs through a controlled debundling process. Also, by doping the surface of CNTs with chemical reagents, a small energy barrier can be created across the CNT-molecule-CNT junctions for optimizing the electronic transport to enhance the overall power factor. Here, the obtained thermoelectric properties were high enough to produce a voltage of $465 \mathrm{mV}$ at $\Delta T=49 \mathrm{~K}$ and output power of $1.8 \mu \mathrm{W}$. They successfully operated a glucose sensor with the help of this TEG, as shown in Fig. 5.

Further, to suppress the effect of contact resistance due to the presence of metal connectors on CNT-based TEGs, a purely carbon-based flexible and lightweight thermoelectric generator was framed by Choi et al. ${ }^{20}$ using carbon nanotube yarn (CNTY) without any additional metal connector. The CNTY was alternately doped with n-type polyethylenimine and p-type $\mathrm{FeCl}_{3}$ to obtain a p-n TE. The highly conductive CNTY space in the doped zone was utilized as an electrical connection to reduce the contact resistance in the TE module. The CNT was synthesized by a floating catalyst method, where ferrocene was used as a catalyst precursor, thiophene as a promoter, and methane as a carbon source at $1200{ }^{\circ} \mathrm{C}$. The CNTY was fabricated by continuously withdrawing aerogels of yarn from the bottom of the reactor wound on a roller and passing the roller through a water bath, which contained hundreds of threads. The as-produced CNTY showed high electrical conductivity of nearly $3147 \mathrm{~S} \mathrm{~cm}^{-1}$ due to the highly aligned structure of the CNT yarn, which resulted in increased longitudinal carrier mobility. Therefore, the flexible TEG containing 60 pairs of p-type- and n-type-doped CNTY exhibited a maximum power density of $10.85 \mu \mathrm{W} \mathrm{g}{ }^{-1}$ and $697 \mu \mathrm{W} \mathrm{g}^{-1}$, and output voltage density of $0.15 \mathrm{~V} \mathrm{~g}^{-1}$ and $1.2 \mathrm{~V} \mathrm{~g}^{-1}$ at $\Delta T$ of $5 \mathrm{~K}$ and $40 \mathrm{~K}$, respectively. Additionally, a red LED was powered by the as-fabricated flexible TEG containing 240 PN pairs at $\Delta T$ of $50 \mathrm{~K}$, as demonstrated in Fig. 6. 

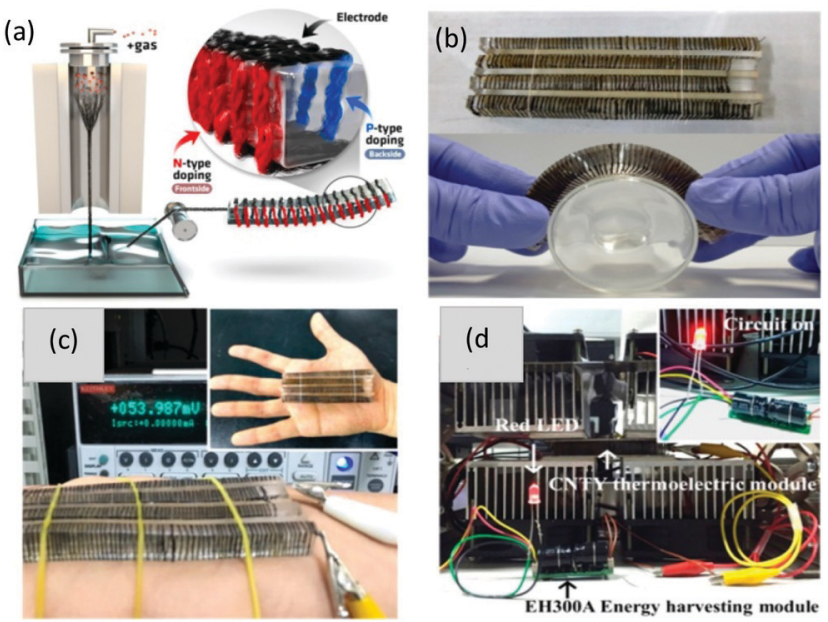

Fig. 6 (a) Fabrication process of the CNTY and flexible TE device. (b) Picture showing the flexibility of the fabricated TEG. (c) Output voltage obtained from the TEG using body heat. (d) Image of a red LED powered using the flexible TEG at $\Delta T=50 \mathrm{~K}$. Reprinted with permission from ref. 20 (J. Choi, Y. Jung, S. J. Yang, J. Y. Oh, J. Oh, K. Jo, J. G. Son, S. E. Moon, C. R. Park, H. Kim, 11 (2017) 7608-7614). Copyright (2017) ACS Publications.

Although good TE performances were validated by doped CNT webs, still only a few studies investigated other carbonbased matrices such as single-walled and double-walled CNTs and graphene. Presently, the two major difficulties in fabricating thin film devices are the use of traditional deposition techniques and maintaining 3-D structural design for mechanical flexibility. To overcome these issues, Ito et $a l^{118}$ presented a wearable flexible device based on single-walled carbon nanotubes (SWCNTs). In this study, the authors introduced a new enhanced direct-injection pyrolytic (eDIP) method for the synthesis of singlewall CNT doped with p-type and n-type dopants. Using this process, they reported the preparation of a fabric-based thermoelectric generator (TEG) by assembling carbon nanotube composite threads. The device could even be used for low-temperature energy harvesting. The CNT threads (p-type) were prepared by dispersing CNTs in an aqueous solution of sodium dodecyl sulfate (SDS), then by adding $0.01 \mathrm{wt} \%$ polyethylene glycol (PEG) using a homogenizer. Further, methanol was added as a coagulant and the wet thread was pulled out of the vessel for air drying. The n-type CNT thread was prepared by partially doping the CNT/PEG thread with 1-butyl-3-methylimidazolium hexafluorophosphate ([BMIM $] \mathrm{PF}_{6}$ ) consisting $10 \mathrm{wt} \%$ of dimethyl sulfoxide (DMSO) as a doping agent, and after immersion for $24 \mathrm{~h}$ in the doping agent, the tread was wiped off with a cotton ball. Here, the obtained Seebeck coefficient for the $\mathrm{p}-\mathrm{n} \mathrm{CNT}$ thread was $101.5 \mu \mathrm{V} \mathrm{K}{ }^{-1}$. The generated output voltage and power were $2.3 \mathrm{mV}$ at $\Delta T$ of $5 \mathrm{~K}$ and $1.6 \mathrm{~mW} \mathrm{~m}^{-2}$ for fifty single threads, respectively. Even after 160 cycles of bending-stretching test on the sample, with a nearly zero inner radius of curvature, no change was observed in resistance.

Conversely, to address the lack of exceptional performances of n-type TE materials, Cho et al. ${ }^{119}$ explored a highly ordered 3-D conjugated network of n-type organic TE nanocomposite by depositing alternate layers of polyethyleneimine (PEI)-stabilized double walled-nanotubes (DWNT) and polyvinylpyrrolidone
(PVP)-stabilized graphene utilizing the layer-by-layer (LbL) deposition method. They prepared a DWNT and graphene solution by adding $0.03 \mathrm{wt} \%$ of DWNT and $0.2 \mathrm{wt} \%$ of graphene in deionized (DI) water comprising $1 \mathrm{wt} \%$ PEI and $0.1 \mathrm{wt} \%$ of PVP, respectively. Later, both solutions were homogenized using a tip sonicator followed by bath sonication. The solutions were centrifuged, and the supernatants were collected for the preparation of thin films of n-type nanocomposite. Through a robotic system, thin n-type TE films were prepared by alternately dipping the PET substrate into the graphene-PVP and DWNT-PEI solutions followed by rinsing with DI water. The graphene and DWNT present in the nanocomposite formed an interconnected network between the upper and lower layer of the films. These bridges helped in transporting electrons more efficiently, hence enhancing the Seebeck coefficient due to increased carrier mobility. In addition, the randomly oriented graphene nanoplatelets, which were aligned parallel to the substrate, had obstructed the penetration of oxygen into the film, endowing the film with environmental stability. Thus, the n-type film demonstrated electrical conductivity of $\sim 300 \mathrm{~S} \mathrm{~cm}^{-1}$, Seebeck coefficient of $-80 \mu \mathrm{V} \mathrm{K} \mathrm{K}^{-1}$ at $80 \mathrm{BL}$ and power factor of $190 \mu \mathrm{W} \mathrm{mK}{ }^{-2}$ at room temperature. Fig. 7 shows the process of the LBL technique and formation of the CNT thread, with the functioning of the wearable TEG by the heat of a human fingertip.

After scanning all the above-reported data of carbon-based TE materials, we concluded that the main reason for obtaining better TE properties from the CNT web is the quasi 1-D

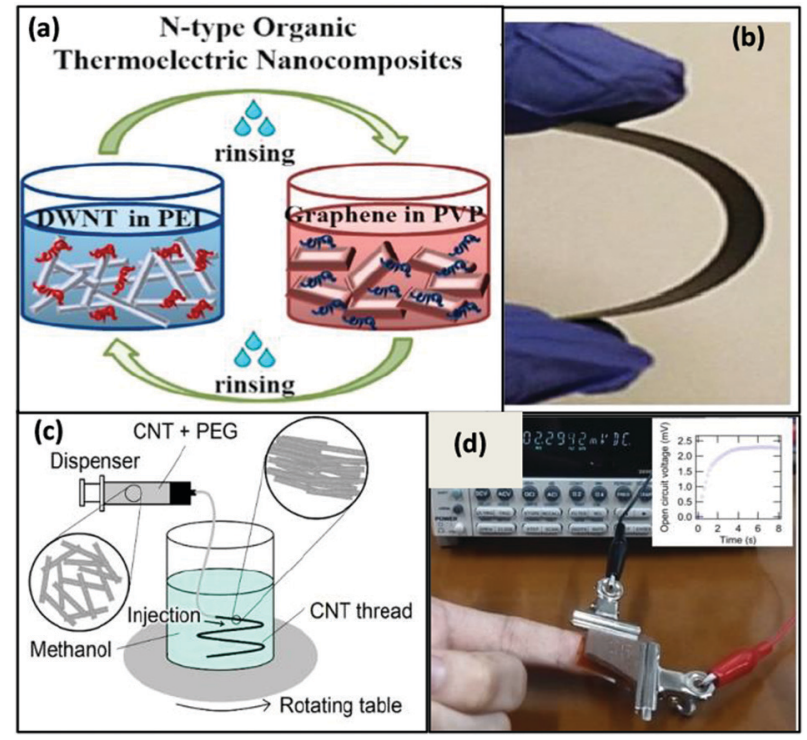

Fig. 7 (a) Schematic of the layer-by-layer deposition process and molecular structures of the materials used and (b) photo of a fully dried, flexible multilayer thin film on PET. Reprinted from ref. 119 (C. Cho, M. Culebras, K. L. Wallace, Y. Song, K. Holder, J. H. Hsu, C. Yu, J. C. Grunlan, Nano Energy, 28 (2016) 426-432) with permission from Science Elsevier, Copyright 2016. (c) Schematic of the wet-spinning process to prepare CNT thread, and (d) image showing power generation by a finger touch and natural air cooling, with inset showing graph of open circuit voltage vs. temperature. Reproduced from ref. 118 with permission from RSC Publications, Copyright 2017 
Table 2 Data compilation of organic/inorganic dopants in carbon-based TE materials

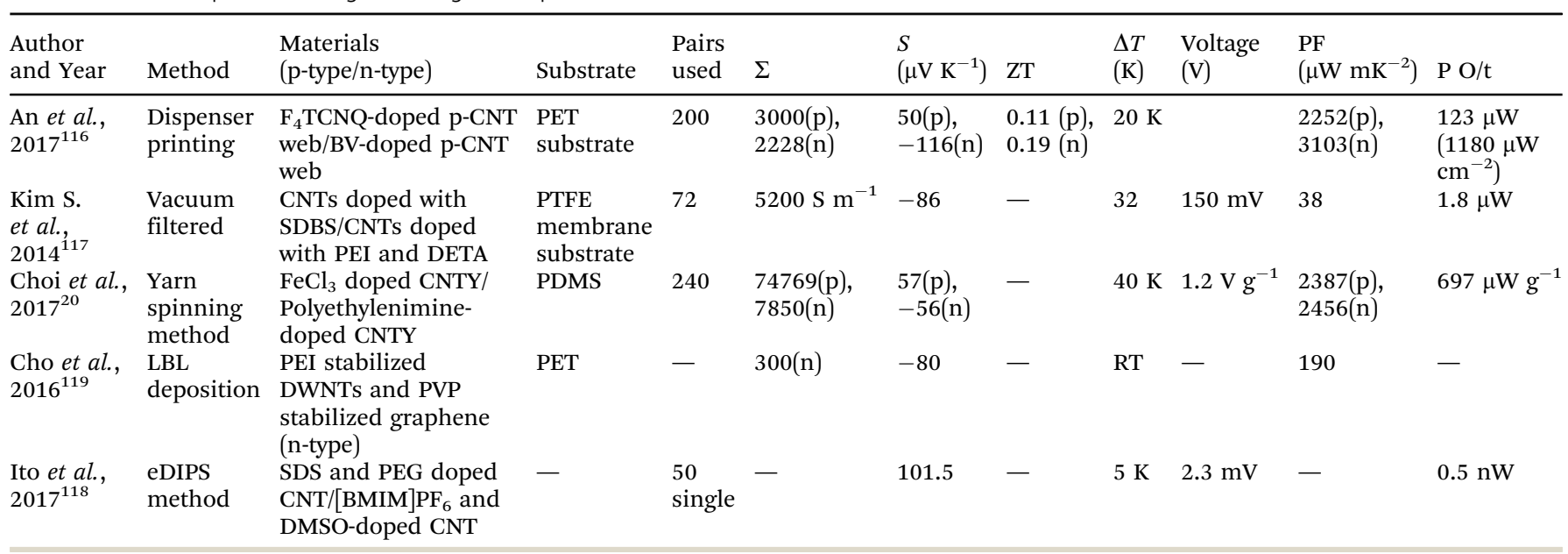

nanostructure of CNTs and suitable doping. The quasi 1-D nanostructure and doping affect the band gap between the conduction and valence band in CNTs. The CNT yarn-based TE devices showed excellent power output, higher flexibility, and less bending cyclic resistance because of the highly aligned yarn structures, resulting in promoted longitudinal carrier mobility. Although the use of 2-D planer nanostructures of graphene is suitable for harsh environmental conditions, it has to compensate for the TE performance. A compilation of the data for organic/inorganic dopants in carbon-based TE materials is presented in Table 2 .

\subsection{Binary nanocomposite- based TE materials}

Besides single organic-based TE, a new class of materials, i.e., binary nanocomposite-based TEGs, has also been investigated by researchers. The reported combinations of binary-based nanocomposites are mostly organic materials (graphene/ CNTs)/conducting polymer (PEDOT:PSS/PANI/PS) and conducting polymer/inorganic nanoparticles.

Graphene/CNT and inorganic material-based combinations have not been investigated widely, possibly because of the high electrical and thermal conductivity of both materials, which can result in a reduced performance in TEGs. Here, we emphasize mainly organic/conducting polymer and conducting polymer/inorganic nanoparticle-based TE nanocomposites prepared by novel methods.

3.2.1 Conducting polymer/CNT/graphene-based binary TE materials. Towards the advancement of nanocomposite formation, graphene and CNTs are the best candidates as a filler material in polymer matrices. However, the major obstacle in the progress of $\mathrm{CNT} /$ polymer composites is regulating the dispersion of CNTs within the polymer matrix. Interestingly, dispersing carbon nanotubes (CNTs) in polyvinyl acetate (PVAc) latex can enhance the electrical conductivity by keeping the Seebeck coefficient and thermal conductivity values relatively constant due to the formation of electrically connected but thermally disconnected bridges between the CNT junctions. ${ }^{120}$ A small energy barrier for the transportation of electrons across the junctions can be significantly tuned by altering the stabilizers.
These stabilizers impart dispersion and exfoliation ${ }^{121,122}$ in CNTs due to van der Waals force, which form natural bundles between them. Theoretically, various strategies such as tuning the electronic states of CNTs, inter-particle distances, potential barriers, electrostatic charges of CNTs and matrices, have been established by researchers to improve the electron transport across the junctions. ${ }^{123,124}$ Here, the stabilizers required for dispersing CNTs in the polymer matrix include surfactants, ${ }^{125,126}$ inorganic nanoparticles, ${ }^{127,128}$ polymers, ${ }^{129,130}$ and biomolecules. ${ }^{131,132}$ Most of the stabilizing agents act as electrical insulators to inhibit electrical transport across the CNT junctions. For example, the dispersion of single-walled carbon nanotubes (SWCNTs) and their stabilization in water were achieved by introducing the intrinsic conducting polymer PEDOT:PSS. Here, the conducting polymer stimulated the electrical conductivity by hampering the settling and aggregation of SWCNTs. ${ }^{124}$ Further, the electrical conductivity of the PEDOT:PSS conducting polymer can be enhanced by doping it with DMSO solvent. ${ }^{133}$ Thus, based on these facts, Grunlan et al. ${ }^{110}$ described the modification of CNT junction by filling DMSO-doped poly(3,4-ethylenedioxythiophene) poly(styrene sulfonate) (PEDOT:PSS) on the surface of CNTs, which resulted in enhanced TE properties. When the PEDOT:PSS particles were decorated on the surface of carbon nanotubes, electrical bridges were formed between the CNTs, which greatly reduced the thermal transport through carriers. Further, this procedure yielded high electrical conductivity $\left(\sim 4000 \mathrm{~S} \mathrm{~m}^{-1}\right)$ without altering the thermopower of the thermoelectric material (TEM). Additionally, the influence of various parameters such as type of CNTs with varying concentrations, stabilizers, and different drying temperatures on the thermoelectric properties was investigated. However, for a CNT concentration of $2-15 \mathrm{wt} \%$ with CNT to PEDOT: PSS ratios of $1: 1-1: 4$, the observed thermal conductivities were similar to that of typical polymeric materials $\left(0.2-0.4 \mathrm{~W} \mathrm{~m}^{-1} \mathrm{~K}^{-1}\right)$. The highest ZT value of 0.02 , Seebeck coefficient of $\sim 26 \mu \mathrm{V} \mathrm{K}{ }^{-1}$, thermal conductivity of $\sim 0.4 \mathrm{~W} \mathrm{~m}^{-1} \mathrm{~K}^{-1}$, and power factor of $\sim 25 \mu \mathrm{W} \mathrm{mK}{ }^{-2}$ were achieved with $35 \mathrm{wt} \%$ SWCNT and $35 \mathrm{wt} \%$ PEDOT: PSS in a copolymer latex-composed nanocomposite matrix. Later, it was dried at RT, and subsequently at a temperature of $80{ }^{\circ} \mathrm{C}$. Similarly, Meng et al. ${ }^{134}$ produced carbon nanotube (CNT) 
and polyaniline (PANI)-based nanocomposites as TE materials. Considering that nanocomposites possess a low dimensional network structure, they demonstrate better TE properties than their parental components. Accordingly, the CNT/PANI nanocomposites were firstly synthesized by filtering a homogenous CNT suspension using a microporous membrane with the assistance of vacuum to prepare a thick CNT network, and secondly by including in situ chemical polymerization of PANI on the CNT network. Afterwards, by adding different concentrations of aniline, various composites were formed and the effect of aniline was examined on the properties of the TE nanocomposites. The outcome demonstrated that on increasing the PANI content in the nanocomposite, the electrical and thermal conductivity decreased, and the Seebeck coefficient and power factor initially increased, but decreased after a certain concentration of PANI. Furthermore, the effect of different CNT sources such as CNT sheets, CNT pellets, and CNT arrays on the TE properties was also studied. The PANI-coated CNT arrays exhibited a higher Seebeck coefficient value than the PANI-coated CNT pellets and sheets.

The maximum values obtained at room temperature with $15.8 \mathrm{wt} \%$ PANI content were a Seebeck coefficient of $28.6 \mu \mathrm{V} \mathrm{K}^{-1}$, electrical conductivity of $6147 \mathrm{~S} \mathrm{~m}^{-1}$, and power factor of $5.0433 \mu \mathrm{W} \mathrm{m}{ }^{-1} \mathrm{~K}^{-1}$ at $300 \mathrm{~K}$. The obtained values were four times that of the CNT sheets and $10^{4}$ times that of PANI. Yao et $a .^{135}$ prepared a nanocomposite of single-walled carbon nanotube (SWNT) and ordered polyaniline via the in situ polymerization of aniline on an SWNT template. Considering that SWNT show superior electrical properties to multi-walled carbon nanotubes (MWCNT) due to their small amount of structural defects, the thermoelectric module performance can be increased by increasing both the Seebeck coefficient and electrical conductivity, which is only possible by increasing the carrier mobility in the material. However, in most conducting polymers, the carrier transport is governed by the inter-chain and intra-chain hopping process, and the arrangement and conformation of the conducting polymer chains affect the carrier mobility. Therefore, it is necessary to develop an ordered chain arrangement, which reduces the barrier for the inter-chain and intra-chain hopping process and increases the carrier mobility, ultimately enhancing the TE performance. Here, the authors used a template- directed synthetic method for the preparation of ordered 1D structures, in which the reactants were located within the templates. The results obtained by TEM, SEM, XRD, and Raman analysis showed that the PANI within the composite was more ordered compared to pure PANI, which is attributed to the substantial $\pi-\pi$ interaction between the PANI molecules and SWNT. The increased Seebeck coefficient at $41.4 \mathrm{wt} \%$ SWNT content was $40 \mu \mathrm{V} \mathrm{K}^{-1}$, and the electrical conductivity was $1.24 \times 10^{4} \mathrm{~S} \mathrm{~m}^{-1}$. The maximum power factor obtained with this composition was $2 \times 10^{-5} \mathrm{~W} \mathrm{~m}^{-1} \mathrm{~K}^{-2}$, and the ZT value reached 0.004 at RT, which was 2 times larger than that of the pure polyaniline.

$\mathrm{Yu}$ et $a{ }^{136}$ reported another light-weight, flexible carbon nanotube/PEDOT:PSS or polyvinyl acetate-based organic composite with high thermoelectric power factors. This group demonstrated that without incorporating highly concentrated binders such as polyvinyl acetate (PVAc) in composites, the CNT concentration could be increased by up to $75 \mathrm{wt} \%$. These hybrid composites were prepared by mixing SWNT in a PEDOT: PSS matrix and/or PVAc emulsion using a homogenizer. Here, PEDOT:PSS acted as a stabilizer, which stabilized the dispersion of nanotubes in water. Different ratios of nanotubes to stabilizers in weight percentage were employed to demonstrate the effect of the corresponding concentration on thermoelectric properties. The highest electrical conductivity and modest Seebeck coefficient recorded (in-plane direction) were $105 \mathrm{~S} \mathrm{~m}^{-1}$ and $41 \mu \mathrm{V} \mathrm{K}^{-1}$, respectively, at RT. Therefore, an optimal nanotube concentration of $60 \mathrm{wt} \%$ resulted in the highest power factor of $160 \mu \mathrm{W} \mathrm{mK}{ }^{-2}$, which is higher than that of the typical polymer composites. The highest thermal conductivity measured in the out of plane direction was $0.2-0.4 \mathrm{~W} \mathrm{~m}^{-1} \mathrm{~K}^{-1}$ at RT. Additionally, a possible schematic of the formation of a junction between CNT and PEDOT:PSS was presented by authors, as shown in Fig. 8(a) with a photograph of the free-standing flexible film in Fig. 8(b). Thus, this method can be beneficial for the manufacture of lightweight, low cost, and safer organic composites for efficient thermoelectric generators. Generally, a reduced thermal conductivity in the composites is a consequence of high surface area and strong van der Waals attractive forces between the CNTs and polymer molecules due to the formation of nanotube aggregates. $^{137}$ The high specific surface area and high thermal conductivity of single-walled CNTs enables more interfaces between crystalline polymers/amorphous nanoparticles to reduce the thermal conductivity by increasing phonon scattering. ${ }^{138,139}$
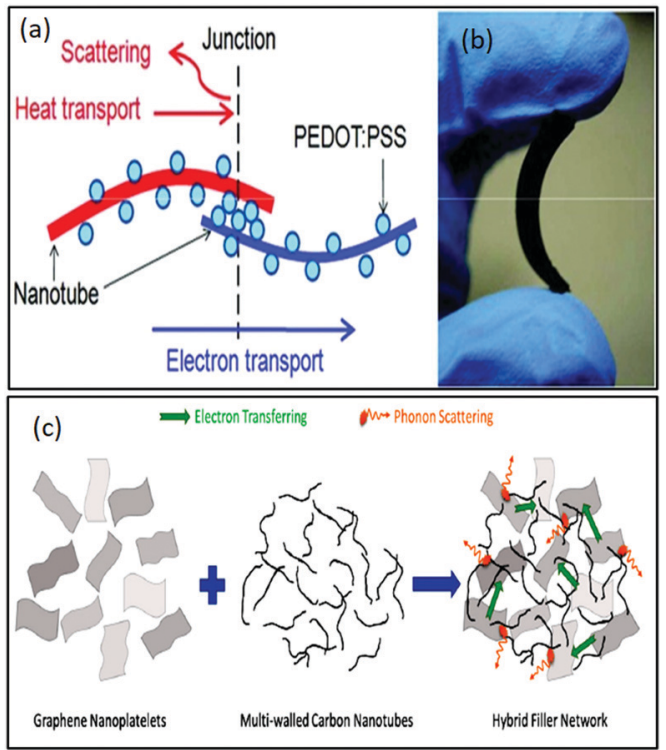

Fig. 8 (a) Schematic of the junction formation between a CNT and PEDOT:PSS for increasing phonon scattering and (b) image of a freestanding flexible film prepared from the composite. Reprinted with permission from ref. 136 (C. Yu, K. Choi, L. Yin, J. C. Grunlan, ACS Nano, 7 (2013) 9506-9506). Copyright (2013) ACS Publications. (c) Schematic of a hybrid 3-D network for transferring electrons and phonons by combining $1 \mathrm{D}$ and 2D conducting nanoparticle effects. Reprinted with permission from ref. 140 (M. Aghelinejad, S. N. Leung, Materials (Basel), 11 (2018) 1-13), Copyright Authors 2018, Open access MDPI. 
Aghelinejad et al. ${ }^{140}$ reported the fabrication of light-weight, flexible, low-cost TE polymer nanocomposite foams using the multilayered network of graphene nanoplatelets (GnPs) and multi-walled carbon nanotubes (MWCNTs) in macroporous foam templates of polyvinylidene fluoride (PVDF). The PVDF macroporous foam templates were synthesized via a saltleaching method followed by drying the disc-shaped molds in an oven. The layer-by-layer (LBL) processing method was employed to deposit the layers of GnPs and MWCNTs on open cellular structures of foam templates, which furnished a platform to mold segregated 3D networks of 1-D and 2-D carbon nanoparticles. Here, the aqueous solution of GnPs was formulated using sodium dodecyl sulfate (SDS) as a surfactant to strengthen the stable dispersion of GnP in the solvent for a mass ratio equal to 1 . Furthermore, various solutions of MWCNT-GnP mixtures were formulated with $0.1 \mathrm{wt} \%$ to $1 \mathrm{wt} \%$ filler content in the hybrid nanocomposite. This hybrid nanocomposite (Fig. 8(c)) provided enhanced electrical conductivity to the system due to the synergetic effect of the GnP and MWCNT 3-D network, and reduced thermal conductivity due to the highly porous structure of the polymer foam substrate. The calculated figure of merit ZT was $10^{-3}$ using a GnP loading of $1.5 \mathrm{vol} \%$ in the macroporous PVDF foam substrate. The highest Seebeck coefficient obtained for the PVDF-GnP nanocomposite was $37 \mu \mathrm{V} \mathrm{K}^{-1}$. According to the authors, this is the highest reported $\mathrm{ZT}$ value for a nanocomposite using a non-conducting polymer and MWCNT/GnP as a nanofiller. Accordingly, Suemori et al. ${ }^{141}$ described a flexible and light-weight TEG composed of carbon nanotube (single-walled carbon nanotubes) and polystyrene composites on a substrate (polyethylene naphthalate film) utilizing a printing method. Here, the used composite material, which contained nearly $35 \mathrm{vol} \%$ of voids, reduced the CNT-polystyrene composite density and resulted in a lightweight TEG (weight/unit area: $15.1 \mathrm{mg} \mathrm{cm}^{-2}$ ). This TEG only consisted of a p-type lightweight material because the n-type lightweight material showed a lower performance. Each thermoelectric leg of the CNT-polystyrene composite (CNT 75 wt\%) was sandwiched between top and bottom electrodes. A composite solution was made by dispersing CNTs and dissolving polystyrene in 1,2-dichlorobenzene. Then, the polystyrene-CNT composite was deposited on the substrate by a printing plate containing holes, which acted like a template. After drying the pattern, the template was removed, and a gold electrode was deposited via the vacuum deposition method on top of it. The cross electrical connection was provided from the top of each leg to the bottom of the next leg, and thus connected serially. The TEG contained 1985 legs of p-type composite (Fig. 9) and generated an output voltage, current, and power density of $254 \mathrm{~V} \mathrm{~m}^{-2}, 0.86 \mathrm{~mA}$, and $55 \mathrm{~mW} \mathrm{~m}^{-2}$, respectively, at $\Delta T$ of $70^{\circ} \mathrm{C}$. The Seebeck coefficient $(S)$ and electrical conductivity of the CNT-polystyrene composite were $57 \mu \mathrm{V} \mathrm{K}^{-1}$ and $2.1 \mathrm{~S} \mathrm{~cm}^{-1}$, respectively.

Therefore, the calculated PF was $0.15 \mu \mathrm{W} \mathrm{m}^{-2} \mathrm{~K}^{-1}$. Considering that $40 \%$ area of the TEG, including the space occupied by the substrate and electrodes, did not contribute to thermoelectric conversion of heat to power output, the overall efficiency of the device was consequently lower. Thus, be

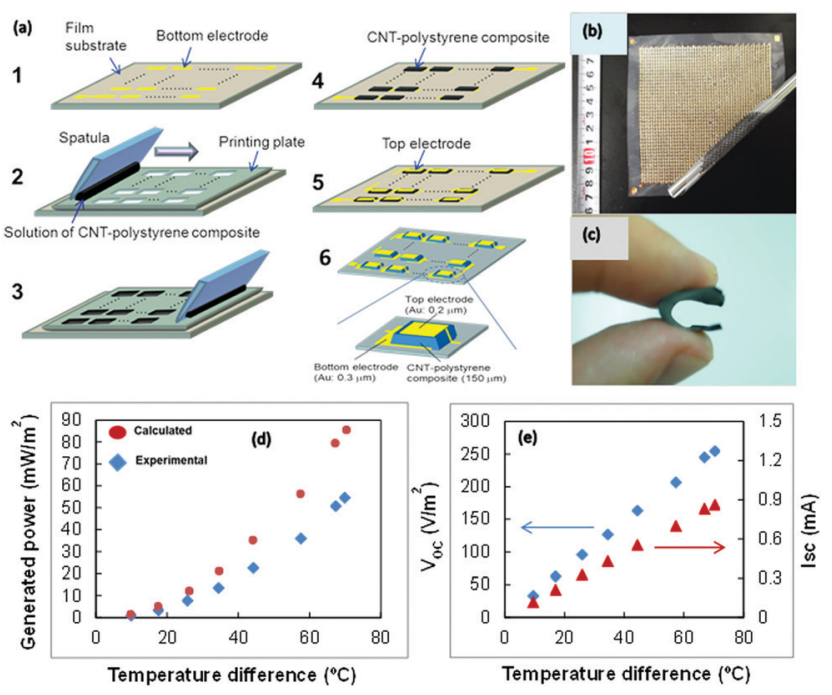

Fig. 9 (a) Schematic of the fabrication steps, (b) photograph of a CNTpolystyrene composite film (thickness: $75 \mu \mathrm{m}$ ) fabricated on a film substrate, (c) photograph of the fabricated TEG, (d) graph of the generated power output, and (e) open circuit voltage and short-circuit current with temperature difference. Reproduced from ref. 141 (K. Suemori, S. Hoshino, T. Kamata, Appl. Phys. Lett. 103 (2013)) with the permission of AIP Publishing.

optimizing the ratio between the area of the CNT-PS composite to the whole area of the TEG, the performance of this type of TEG can be improved to around $50 \%$.

Similarly, Hewitt et al. ${ }^{142}$ fabricated a flexible TEG containing 72 individual layers of CNT and PVDF. Here, the CNT and PVDF layers were used as conducting and insulating layers, respectively. The CNT and PVDF individual films were placed alternately, and then the stack was pressed together to form a felt fabric at the polymer melting point. For the 72-layer CNT/PVDF thermoelectric, the obtained output voltage and power were $26 \mathrm{mV}$ and $137 \mathrm{nW}$ for a $\Delta T$ of $50 \mathrm{~K}$, which is quite low, respectively. Additionally, the obtained Seebeck coefficient was $550 \mu \mathrm{V} \mathrm{K}{ }^{-1}$ at the $\Delta T$ of $95 \mathrm{~K}$. Kim et al. ${ }^{143}$ reported that the addition of $2 \mathrm{wt} \%$ graphene on conducting polymer poly(3,4-ethylenedioxythiophene):poly(styrenesulfonate) (PEDOT:PSS) thin films could improve their thermoelectric properties by 10 times. In contrast, to achieve the same result, 30-40 wt\% of CNT was required. Further, when graphene was incorporated into the conducting polymer matrix, the interfacial area associated with the molecules increased by a factor of 2-10 compared to CNT molecules for the equivalent weight percentage. Nanocomposite thin films were prepared by combining graphene and $2 \mathrm{wt} \%$ PEDOT: PSS solution in isopropyl alcohol/deionized water followed by sonication to form a homogeneous solution. The stronger $\pi-\pi$ interaction between graphene and conducting polymer stimulated their dispersion and increased the carrier concentration by reducing the $\pi-\pi$ conjugated defects in the PEDOT:PSS backbone, as represented by the mechanism shown in Fig. 10. Thereafter, the spin coating technique was used to fabricate a thin film on an $\mathrm{Si} / \mathrm{SiO}_{2}$ substrate, followed by heating the thin film in an oven to remove the solvent. The obtained power factor, Seebeck coefficient, electrical conductivity, 


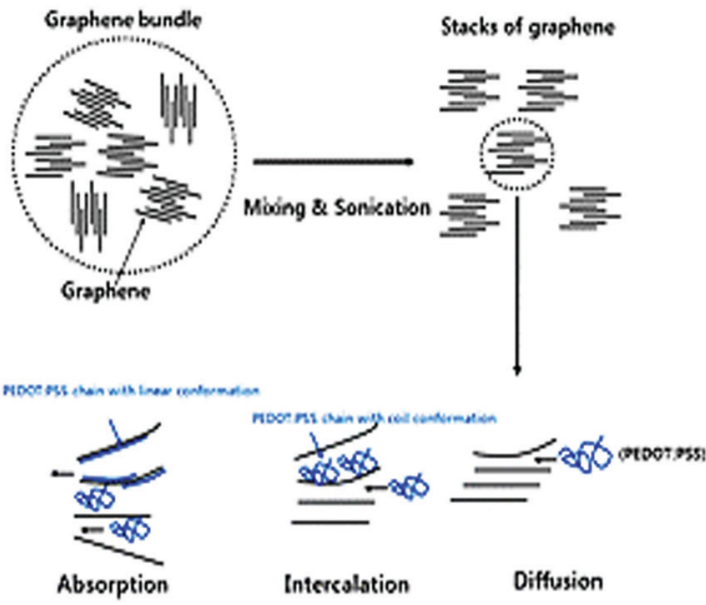

Fig. 10 Mechanism of the dispersion of aggregated graphene in the conducting polymer during the mixing and sonication process. Reproduced from ref. 143 with permission from RSC Publications, Copyright 2012

thermal conductivity, and $\mathrm{ZT}$ value of the nanocomposite thin film were $11.09 \mu \mathrm{W} \mathrm{mK} \mathrm{mK}^{-2}, 58.77 \mu \mathrm{V} \mathrm{K} \mathrm{K}^{-1}, 32.13 \mathrm{~S} \mathrm{~cm}^{-1}$, $0.14 \mathrm{~W} \mathrm{~m}^{-1} \mathrm{~K}^{-1}$ and 0.021 , respectively. Therefore, the higher ZT value was an outcome of the high electron mobility of graphene and porous structure of the thin film, which reduced the thermal conductivity.

Similarly, Wang et al. ${ }^{144}$ reported that the electrical conductivity and thermopower of polyaniline (PANI) could be simultaneously improved by incorporating single- or doublewalled carbon nanotubes (S/DWCNT) in its matrix. Moreover, the electron mobility of PANI was increased by doping it with camphorsulfonic acid (CSA). PANI, PANI-CSA and S/DWCNT solutions were prepared in $m$-cresol using a pen sonicator, separately. Then different ratios of the CNT solution were added to the PANI and PANI-CSA solutions followed by bath sonication to obtain a homogenous mixture. These solutions were dropped onto a glass substrate, dried, and annealed under vacuum to obtain thin films. Further, different composites of PANI/CNT and PANI-CSA/CNT were prepared by varying the concentration of SWCNTs or DWCNT. Consequently, this method showed a 50-fold increment in composite electron mobility from $\sim 0.15$ to $\sim 7.3 \mathrm{~cm}^{2}(\mathrm{~V} \mathrm{~s})^{-1}$, and a sharp decrease by nearly 4 times in the charge carrier concentration from $\sim 2.1 \times 10^{21}$ to $\sim 5.6 \times 10^{20} \mathrm{~cm}^{-3}$. The band alignment of CNT/PANI was responsible for the higher number of hole carriers, which is associated with the high mobility of CNT, enhancing the electrical conductivity and Seebeck coefficient. The composite with $30 \mathrm{wt} \%$ DWCNTs/PANI-CSA demonstrated the highest electrical conductivity of $\sim 610 \mathrm{~S} \mathrm{~cm}^{-1}$, Seebeck coefficient of $\sim 61 \mu \mathrm{V} \mathrm{K}^{-1}$ at RT, and PF of $220 \mu \mathrm{W}\left(\mathrm{m} \mathrm{K}^{2}\right)^{-1}$, which was 2 times larger than that of the PANI-CSA, PANI and PANI/SWCNT composite. Additionally, the composite showed mechanical flexibility, low toxicity, lightweight material, and easy manufacturing process.

Luo et al. ${ }^{145}$ reported the preparation of CNT and block copolymer poly(ether- $b$-amide12) (PEBA) blended TE films, which were utilized for fabricating a flexible TEG. Firstly, PEBA was doped with $\mathrm{LiClO}_{4}$ to prepare a PEBA- $\mathrm{Li}^{+}$solution. Then, an n-type CNT solution was prepared by doping with DETA followed by vacuum filtration and vacuum drying at $45{ }^{\circ} \mathrm{C}$ for $24 \mathrm{~h}$. Next, the $20 \mathrm{wt} \%$ p-type CNT and DETA-doped n-type CNT powders were re-dispersed in formic acid and added to the PEBA-Li ${ }^{+}$solution, then mixed by stirring at $45{ }^{\circ} \mathrm{C}$ for $24 \mathrm{~h}$. The mixed PEBA/CNT p-type and n-type bilayer heterogeneous films were cast on a glass substrate and later peeled off from the substrate after drying for a long period to obtain a heterogeneous structure. The obtained Seebeck coefficient and electrical conductivity for the $\mathrm{p}^{-}$and n-type TE films were $36.88 \mu \mathrm{V} \mathrm{K}^{-1}$ and $-33.25 \mu \mathrm{V} \mathrm{K}{ }^{-1}$, and $456 \mathrm{~S} \mathrm{~m}^{-1}$ and $492 \mathrm{~S} \mathrm{~m}^{-1}$, respectively. Additionally, a flexible TEG was fabricated by connecting the p-type and n-type TE films in series on a polyimide substrate. The TE device with 3 pairs of $\mathrm{p}-\mathrm{n}$ showed an output voltage of $120 \mathrm{mV}$ at a temperature difference of $60 \mathrm{~K}$. Thus, according to all the above-reported data, we observed that the distinct bonding and different vibrational spectra between CNTs and PEDOT:PSS/PANI molecules yielded a factorial enhancement in power factor compared to their parental groups. Additionally, different polymeric molecules can be used as fillers for increasing the power factor by obstructing the direct thermal transport of carriers.

3.2.2. Conducting polymer/inorganic semiconductor-based binary TE materials. The ability to print various nanocompositebased TEM on flexible substrates for wearable applications envisaged development of different printing methods. A fully in situ aerosol jet-printed organic/inorganic nanocompositebased flexible TEG was reported by $\mathrm{Ou}$ and co-workers. ${ }^{25}$ In their work, they synthesized $\mathrm{Bi}_{2} \mathrm{Te}_{3} / \mathrm{Sb}_{2} \mathrm{Te}_{3}$ nanocrystals via the solvothermal method. The low-temperature solution-based process allowed good size and shape control at the nanoscale, which could help in synergistically tuning the TE parameters. $\mathrm{Bi}_{2} \mathrm{Te}_{3} / \mathrm{Sb}_{2} \mathrm{Te}_{3}$ inorganic ink and PEDOT:PSS organic inks were prepared in pneumatic atomizer (PA) and ultrasonic atomizer (UA), respectively. Then, in situ mixing was employed in an aerosol-jet printer and the inks were deposited on a malleable polyimide substrate, as shown in Fig. 11. Based on the loading fraction of the inorganic component in the mixture, various thermoelectric properties and morphologies of the printed structure were observed. The maximum power factor produced with a minimal loading fraction of $85 \mathrm{wt} \% \mathrm{Sb}_{2} \mathrm{Te}_{3}$ nanoflakes was $\sim 30 \mu \mathrm{W} \mathrm{mK}^{-2}$. The best TE properties obtained for this composition was $S \sim 33.8 \mu \mathrm{V} \mathrm{K}^{-1}$ and $\sigma \sim 247.3 \mathrm{~S} \mathrm{~cm}^{-1}$, with superior mechanical properties. Upon repeated flexing on a curvature of up to $300 / \mathrm{m}$, the printed nanocomposite TEG was consistently stable and healthy. The aerosol jet printing technique has several benefits over other printing methods. Thus, AJP is a scalable, large-area deposition, and commercially viable technique for the preparation of electronic materials and custom formulations.

The continuous demand for wearable mobile electronics in the market has evoked the requirement of flexible TEG modules. Thus, We et al. ${ }^{146}$ presented a screen-printed flexible thermoelectric power generator based on a hybrid composite of 
(a)

Pneumatic Atomiser

Ink: $\mathrm{Bi}_{2} \mathrm{Te}_{3} \downarrow \mathrm{N}_{2}$ flow $\mathrm{Sb}_{2} \mathrm{Te}_{3}$

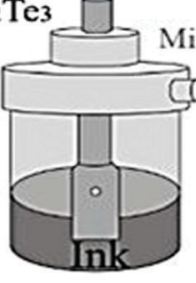
$\rightarrow$ $\longrightarrow$ Qatm

(b)

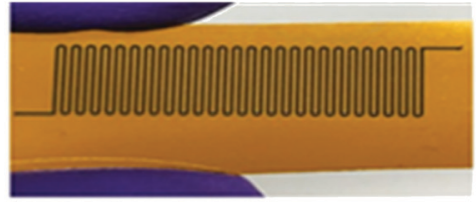

(c)

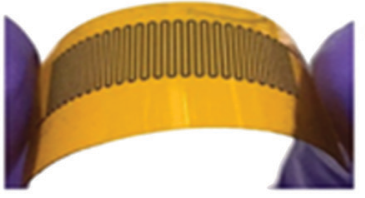

(d)

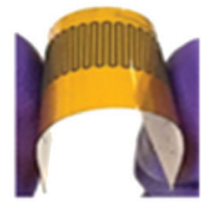

(e)
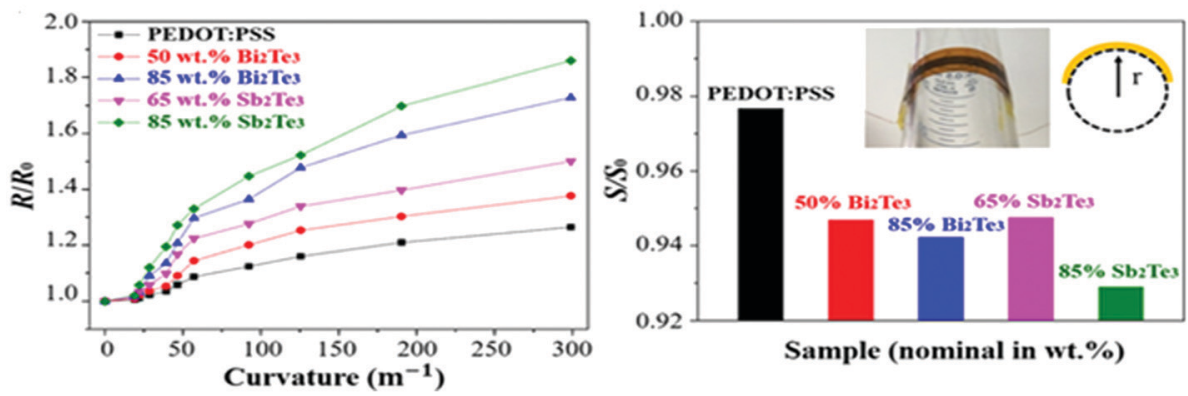

Fig. 11 (a) Schematic of the aerosol jet printing method for preparing nanocomposites of PEDOT:PSS and $\mathrm{Bi}_{2} \mathrm{Se}_{3} / \mathrm{Sb}_{2} \mathrm{Te}_{3}$. Image of flexible material bent to different degrees: (b) flat, (c) lower degree, and (d) higher degree, and (e) flexibility test based on ratio of flat to flexed resistance vs. curvature and ratio of flat to flexed Seebeck coefficient vs loading of semiconducting materials. Reprinted with permission from ref. 25 (C. Ou, A. L. Sangle, A. Datta, Q. Jing, T. Busolo, T. Chalklen, V. Narayan, S. Kar-Narayan, ACS Appl. Mater. Interfaces, 10 (2018) 19580-19587). Copyright (2018), ACS Publications.

inorganic/organic conducting polymer TE film. The inorganic material paste consisted of various weight compositions such as $75 \%$ metal powder $\mathrm{Bi}, \mathrm{Sb}$, and $\mathrm{Te}, 0.2 \%$ binder, $2.4 \%$ glass powder, and $22.4 \%$ solvent. Both pastes were homogeneously blended in ball mill equipment for $24 \mathrm{~h}$. The pastes were screen printed through a 150-mesh screen onto a flexible polyimide film substrate. Afterwards, the samples were dried to remove the solvent, followed by a two-step annealing method. The first step involved the removal of the organic binder under vacuum conditions, and the second step was conducted at a higher temperature under $\mathrm{N}_{2}$ ambient conditions to obtain the best TE properties. After annealing, the (PEDOT:PSS) coating was done, which penetrated through the micropores of the screen-printed bismuth and antimony telluride TE thick films, inducing flexibility in the TE module without affecting its output performance. Ag paste was used to make electrical contacts. Also, the area ratio of $\mathrm{p}$ to $\mathrm{n}$ legs was optimized for maximizing the output power density of the TEG module. The TE legs exhibited different thermal and electrical properties, which affected the figure of merit of the TE module. The relation of the figure of merit was given as: $\mathrm{ZT}=\left(S_{\mathrm{p}}-S_{\mathrm{n}}\right)^{2} / R \cdot K$, where $S_{\mathrm{p}}$ and $S_{\mathrm{n}}$ are the Seebeck coefficient of the p-type and n-type TE material, and $R$ and $K$ represent the resistance and thermal conductance of the TE module, respectively.
The electrical resistance $(R)$ and thermal conductance $(K)$ can be defined as:

$$
R=\frac{L_{\mathrm{p}} \cdot \rho_{\mathrm{p}}}{A_{\mathrm{p}}}+\frac{L_{\mathrm{n}} \cdot \rho_{\mathrm{n}}}{A_{\mathrm{n}}} \quad K=\frac{\kappa_{\mathrm{p}} \cdot A_{\mathrm{p}}}{L_{\mathrm{p}}}+\frac{\kappa_{\mathrm{n}} \cdot A_{\mathrm{n}}}{L_{\mathrm{n}}}
$$

where $L, \rho, A$, and $k$ represent the length, electrical resistivity, area of TE leg, and thermal conductivity, respectively. The maximum value of $\mathrm{ZT}$ can be obtained when the product RK is minimized by differentiating the RK value with respect to $A_{\mathrm{n}} / A_{\mathrm{p}}$ and keeping that value equal to zero.

$$
\mathrm{RK}=\left(\frac{L_{\mathrm{p}} \cdot \rho_{\mathrm{p}}}{A_{\mathrm{p}}}+\frac{L_{\mathrm{n}} \cdot \rho_{\mathrm{n}}}{A_{\mathrm{n}}}\right) \cdot\left(\frac{\kappa_{\mathrm{p}} \cdot A_{\mathrm{p}}}{L_{\mathrm{p}}}+\frac{\kappa_{\mathrm{n}} \cdot A_{\mathrm{n}}}{L_{\mathrm{n}}}\right)
$$

The obtained maximum Seebeck coefficient with an area ratio of 3 for a TEG module was $240.5 \pm 4.2 \mu \mathrm{V} \mathrm{K}^{-1}$. The output voltage and power density produced by TEG for a $\mathrm{p}-\mathrm{n}$ pair of 7 was $85.2 \mathrm{mV}$ and $1.2 \mathrm{~mW} \mathrm{~cm} \mathrm{~cm}^{-2}$ at $\Delta T$ of $50 \mathrm{~K}$ with a hot side temperature of $333 \mathrm{~K}$, respectively. In addition, a flexible TEG module with $15 \mathrm{p}-\mathrm{n}$ pairs was demonstrated using body heat and ambient temperature conditions, showing an output voltage of $12.5 \mathrm{mV}$ at a $\Delta T$ of $5 \mathrm{~K}$. A thermoelectric nanogenerator (TENG) was fabricated by simply mixing Te nanowires and P3HT polymer. The Te nanowires were synthesized by reducing 
$\mathrm{TeO}_{2}$ with hydrazine hydrate at room temperature under constant stirring. When Te nanowires were grown on the surface of Te nanocrystallites by depositing Te atom, the solution exhibited a colour change from colourless to blue at the end of the reaction. For device fabrication, a composite of Te nanowires and poly(3-hexylthiophene) (P3HT) polymer was prepared in benzene chloride solution. The composite solution was dropped on a flexible Kapton substrate and silver paste was used as the electrode. The fabricated TENG was packaged by a thin layer of poly(dimethylsiloxane) (PDMS) to protect it from ambient conditions. The produced output voltage/current was $14 \mathrm{mV} /$ $254 \mathrm{nA}$ at a $\Delta T$ of $50 \mathrm{~K}$. A linear variation between the temperature difference and the output voltage was observed during testing. Yang's group reported that when two are TENGs connected in series, the output voltage of $38 \mathrm{mV}$ and current density of $320 \mathrm{nA}$ in parallel connection at a $\Delta T$ of $55 \mathrm{~K}$ were achieved. The calculated Seebeck coefficient was $285 \mu \mathrm{V} \mathrm{K}^{-1}$. By utilizing the temperature of the human body as a heat source, these thermoelectric nanogenerators (TENGs) could be used as a wearable energy harvester. Additionally, they were used as a self-powered temperature sensor, which displayed a response time of $17 \mathrm{~s}$ and reset time of $9 \mathrm{~s}$. Under ambient conditions, the detection sensitivity of the sensor reached up to $0.15 \mathrm{~K}^{147}$

Wang et al. ${ }^{148}$ reported PEDOT: PSS-based aerogel films and a PEDOT:PSS/TeNW-based composite aerogel film for a flexible thermoelectric power generator because of their high flexibility and ultralow thermal conductivity. With different organic solvents, the PEDOT:PSS aerogel films were cast by vacuum freeze-drying followed by pressing. Similarly, PEDOT:PSS/ TeNW (PPT) films were prepared using the same method, together with the hydrothermal method, which was used for TeNW. Finally, vapour annealing of the PPT composite aerogel films was done with DMSO solvent in a Teflon-lined stainless steel autoclave. The PEDOT:PSS/30 wt\% Te-NW aerogel composite film was treated with DMSO vapour for $20 \mathrm{~min}$, resulting in a PF of $11.3 \mu \mathrm{W} \mathrm{m} \mathrm{m}^{-1} \mathrm{~K}^{-1}$, ZT of $2.0 \times 10^{-2}$, electrical conductivity of $\sim 100 \mathrm{~S} \mathrm{~cm}^{-1}$, and Seebeck coefficient of $\sim 32.1 \mu \mathrm{V} \mathrm{K}^{-1}$. The device was fabricated by connecting the PEDOT:PSS-based aerogel films as the p-type leg and carbon nanotube fibres (CNFs) as the n-type leg in series with silver paste. The maximum output voltage, output current, and output power obtained were $31.2 \mathrm{mV}$, $\sim 0.17 \mathrm{~mA}$ and $1.28 \mu \mathrm{W}$ at $\Delta T$ of $60 \mathrm{~K}$, respectively. The optimized composite aerogel films provided an efficient TE generator with high-performance values. Wang and coworkers ${ }^{149}$ fabricated a highly flexible.

Solution-printable n-type hybrid TE based on a fullerene/ $\mathrm{TiS}_{2}$ organic/inorganic material. $\mathrm{TiS}_{2}$ powder was exfoliated to 2D nanosheets and intercalated with $N$-methyl-2-pyrrolidone (NMP) by grinding it in a mortar-pestle. Then, the powder was sonicated in isopropyl alcohol (IPA) followed by centrifugation to remove the bulk particles. Thereafter, a $\mathrm{C}_{60}$ (fullerene) solution prepared in toluene was added slowly to the $\mathrm{TiS}_{2}$ supernatant in various amounts. The mixing of both solutions provided a path to deposit $\mathrm{C}_{60}$ particles on the surface of nanosheets. Then, the final solution was passed subjected to vacuum filtration with the help of membranes to obtain hybrid flexible films, followed by vacuum drying and annealing. This solution was used as an n-type ink for the fabrication of a TEG. Additionally, the p-type ink was developed by dispersing and sonicating $50 \mathrm{wt} \%$ SWNTs in PEDOT:PSS. Further, by using both inks, a flexible TEG was printed on a plastic substrate. The n-type $\mathrm{C}_{60} / \mathrm{TiS}_{2}$ showed a ZT value of $\sim 0.3$ at $400 \mathrm{~K}$. For $1 \mathrm{wt} \%$ concentration of $\mathrm{C}_{60}$, the obtained thermoelectric properties for the n-type hybrid were a Seebeck coefficient of $-101 \mu \mathrm{V} \mathrm{K} \mathrm{K}^{-1}$, electrical conductivity of $\sim 390 \mathrm{~S} \mathrm{~cm}^{-1}$, and power factor of $\sim$ $400 \mu \mathrm{W} \mathrm{mK}{ }^{-2}$ at $300 \mathrm{~K}$. The obtained data for the flexible TEG was an output voltage of $\sim 4.8 \mathrm{mV}$, maximum power output of $335 \mathrm{nW}$, and power density of $1.68 \mathrm{~W} \mathrm{~m}^{-2}$ at a temperature difference of $20 \mathrm{~K}$. Hence, these hybrid nanocomposites are better than the previously reported nanocomposites because of the higher thermopower of the inorganic particles and low thermal conductivity of the conducting polymer. The novel aerosol jet printing technique can provide a versatile, economic, and easily scalable method for printing flexible nanocomposite-based TEGs. In addition, other factors such as optimization of the TE legs and TE area show great impact on the performance of TEGs. The reported data for binary hybrid nanocomposite-based TE materials is summarized in Table 3.

\subsection{Ternary hybrid nanocomposite-based TE materials}

Even after covering all the binary hybrid nanocomposites, achieving high electrical conductivity in nanocomposites still remains a challenging task. Thus, to overcome this difficulty, the ternary hybrid nanocomposite-based TE materials were combined to obtain the benefit of all the three components in a nanocomposite. A prospective strategy to enhance the Seebeck coefficient and electrical conductivity of hybrid nanocomposites can be developed by integrating higher $S$ inorganic NPs and higher $\sigma$ conducting elements. ${ }^{31,150-153}$ However, the power factor of these hybrids cannot exceed the values of their individual constitutes. Besides, the limitations have been alleviated by providing large energy carriers filtering at hybrid hetrojunctions. ${ }^{154,155}$

Generally, ternary nanocomposites are composed of an organic-based matrix on which surface inorganic nanoparticles and conducting polymers are grown by either physical mixing or in situ polymerization. Therefore, Ou et al. ${ }^{24}$ used a ternary nanocomposite ink as a thermoelectric material with different viscosities and ratios to fabricate flexible TEGs with various substrates using a modified aerosol-jet printing method. Highly dispersible antimony telluride nanoflakes and multi-walled carbon nanotubes (MWCNTs) were incorporated into poly(3,4ethylenedioxythiophene) polystyrene sulphonate (PEDOT:PSS) structures to enlarge the TE properties of the conducting polymer on the flexible polyimide substrates. The PEDOT:PSS ink and MWCNT ink were formulated by dispersing them in DI water and loading in an ultrasonic atomizer and pneumatic atomizer, respectively. To prepare a stable dispersion of MWCNT in DI water, $0.1 \mathrm{wt} \%$ of sodium dodecyl sulphate (SDS), and $0.5 \mathrm{wt} \%$ of polyvinylpyrrolidone (PVP) as surfactants were used. The $\mathrm{Sb}_{2} \mathrm{Te}_{3}$ nanoflakes were prepared via the solvothermal synthesis method and added to the atomizer to 
Table 3 Reported data for binary hybrid nanocomposite-based TE materials

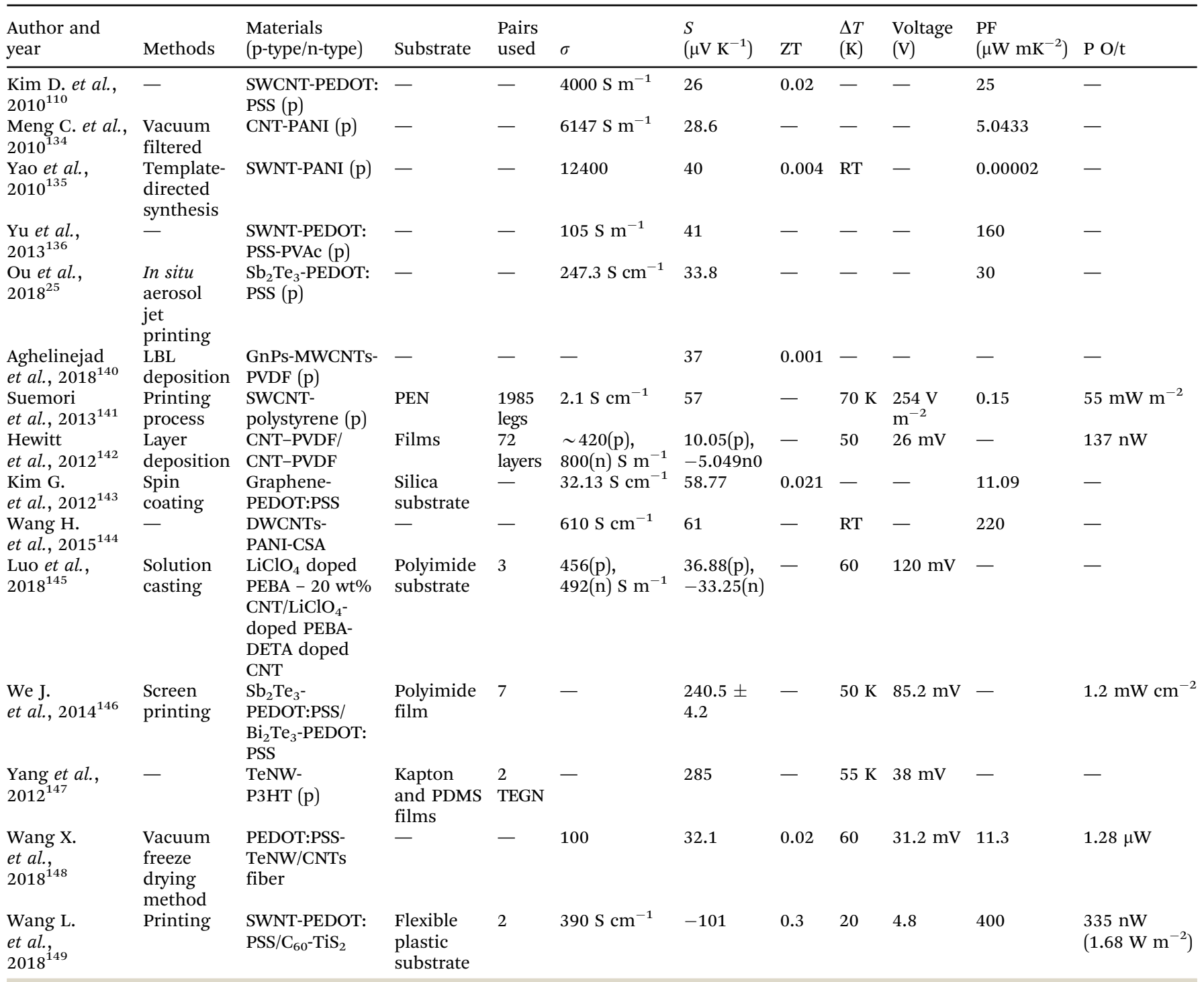

enhance the TE performance. Later, the two different atomized aerosols were in situ mixed to form a stable ink for printing. The higher Seebeck coefficient of the $\mathrm{Sb}_{2} \mathrm{Te}_{3}$ nanoflakes and larger electrical conductivity of MWCNT lowered the thermal conductivi1ty of the nanocomposite by phonon scattering, and significantly enhanced the performance parameter by providing good inter-particle connectivity between the components of the nanocomposites. Further, the electrical conductivity of the nanocomposite device was improved by treating it with different polar solvents, i.e. de-doping of PSS. The minimal loading fraction of $85 \mathrm{wt} \%$ in the $\mathrm{Sb}_{2} \mathrm{Te}_{3}$-MWCNTs-PVP-PEDOT: PSS nanocomposite treated with DMSO showed a power factor of $\sim 41 \mu \mathrm{W} \mathrm{mK}{ }^{-2}$, Seebeck coefficient of $29 \mu \mathrm{V} \mathrm{K}^{-1}$, and electrical conductivity of $496 \mathrm{~S} \mathrm{~cm}^{-1}$. After subjecting the printed TE nanocomposite generator to $60 \mathrm{~h}$ of continuous flexibility testing cycles, the device showed a stable performance and great mechanical strength. Jung et al. ${ }^{156}$ developed a flexible hybrid thermoelectric generator (TEG) based on Bi/Te powder, carbon nanotubes (CNTs) and polydimethyl siloxane (PDMS). When bismuth telluride powder $(\mathrm{Bi} / \mathrm{Te})$ was incorporated into CNTs, it was converted to a p-type and n-type TE material. Here, CNTs were used to increase the thermal conductivity of the TE materials. These TE materials were then blended with PDMS solution and stirred in an overhead stirrer. To obtain a homogeneous distribution of components in the solution, it was ultra-sonicated for $3 \mathrm{~h}$ at a frequency of $40 \mathrm{kHz}$. To strengthen the composite, a hardener was required, in which, the principal material to hardener ratio taken as $10: 1$. The composite was then stirred, ultra-sonicated, and degassed under vacuum conditions. Here, PDMS was also used as the substrate to fabricate a flexible TEG. Moreover, to reduce the contact resistances between the thermoelectric material and electrodes, it was selectively etched from the top and bottom of the PDMS surfaces. The recorded Seebeck coefficients for the p-type and n-type TE materials were 143 and $-174 \mathrm{mV} \mathrm{K}^{-1}$, respectively. The generated output voltage and power was $920 \mathrm{mV}$ and 


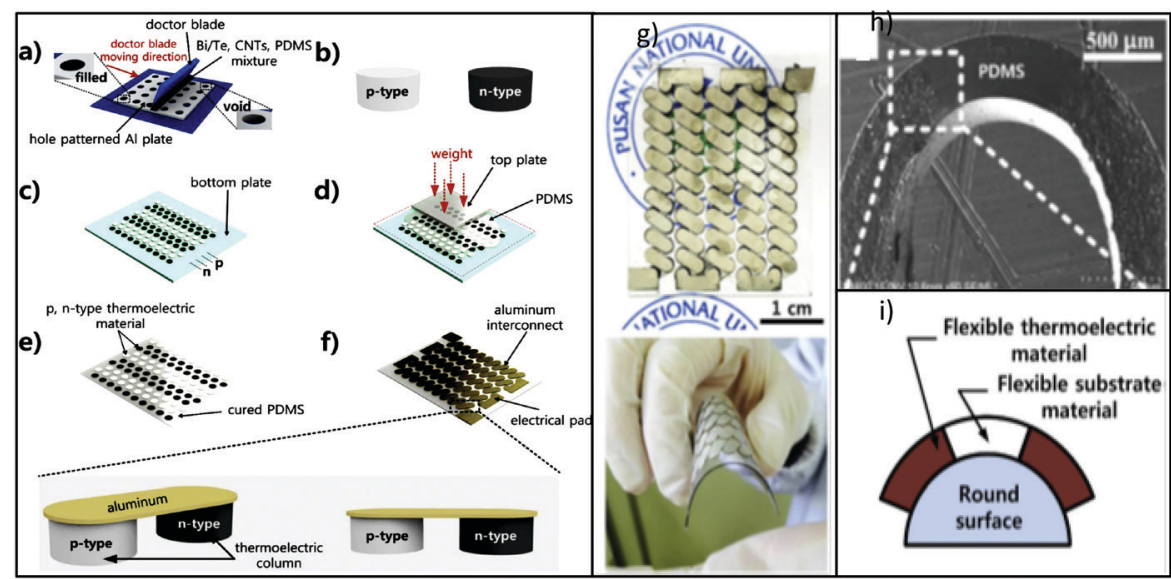

Fig. 12 (a) Fabrication of a single thermoelectric cell, (b) p- and n-type TE unit cell structure, (c) unit cells arranged on a plate, (d) filling of PDMS between the space of unit cells and top and bottom plates, (e) cured PDMS material separated from the top and bottom plates, ( $f$ ) etched PDMS surface and deposition of aluminium interconnect on top and bottom of TEG, (g) photograph of fabricated TEG, (h) cross-sectional SEM image of the fabricated TEG, showing the curved shape of the thermoelectric material, PDMS substrate, and junction area; and (i) flexible thermoelectric materials on a round surface. Reprinted from ref. 156 (K. K. Jung, Y. Jung, C. J. Choi, J. M. Lee, J. S. Ko, Curr. Appl. Phys. 16 (2016) 1442-1448), with permission from Science Elsevier, Copyright 2016. (d).

$570 \mathrm{~mW} \mathrm{~cm}^{-2}$ at a $\Delta T$ of $60{ }^{\circ} \mathrm{C}$. Even during a bending reliability test on a small curvature radius of $5 \mathrm{~mm}$ and $20 \mathrm{~mm}$ over continuous repetition, the TEG maintained its reliability and stability, as shown in Fig. 12 . Choi et al. ${ }^{157}$ reported a highly flexible, mechanically, and chemically durable power conversion device using a ternary hybrid nanocomposite of graphene/polymer/inorganic nanocrystal on a paper substrate with higher performance. Te nanowires (TeNW) were used as inorganic nanocrystals and PEDOT:PSS as the conducting polymer. The hybrid nanocomposite showed two hetero-junctions of rGO/PEDOT:PSS and PEDOT:PSS/TeNW, which stimulated the double carrier filtering of low energy carriers by scattering, and ultimately increased the electrical conductivity without sacrificing a considerable loss in the thermopower of the nanocomposite. PEDOT:PSS-coated TeNW (DTe) was synthesized via a surfactant-assisted chemical reduction method. This method utilized a prepared clear mixture of L-ascorbic acid and dimethyl sulfoxide in DI water, followed by the addition of PEDOT:PSS solution, and $\mathrm{Na}_{2} \mathrm{TeO}_{3}$ under continuous stirring unit a white suspension was obtained. To obtain a precipitate, the suspension was heated to $90^{\circ} \mathrm{C}$ followed by natural cooling to $25{ }^{\circ} \mathrm{C}$. Finally, the product was recovered by centrifugation followed by washing several times with DI water and ethanol and freeze-drying for 2 days. A homogenous suspension of GO-DTe was prepared by re-dispersing the GO-DTe powder in DI water, followed by sonication. The films were prepared by passing the GO-DTe suspension through anodic membrane filters by applying vacuum filtration. Then, the films were delicately exfoliated from the membrane and dried by air and vacuum. The $\mathrm{G}_{0.1} \mathrm{DTe}_{0.9}$ hybrid papers were fabricated by treating the GO-DTe films with HI vapour-phase for chemical graphitization of the GO phase, the as shown in Fig. 13. The reported TE properties were ZT of $\sim 0.21$, power factor of $143 \mu \mathrm{W} \mathrm{m}^{-1} \mathrm{~K}^{-1}$ at $300 \mathrm{~K}$, electrical conductivity of $3496 \mathrm{~S} \mathrm{~m}^{-1}$, thermopower of $202 \mu \mathrm{V} \mathrm{K}^{-1}$, and produced output voltage of $58 \mathrm{mV}$ at $\Delta T$ of $50 \mathrm{~K}$.
Polyethyleneimine (PEI)-doped single-walled carbon nanotubes (SWCNTs) were used as an n-type material and hybrid nanocomposite paper as a p-type material to fabricate a flexible power generator. Thus, for a pair of 5 legs, the generated maximal power density was $650 \mathrm{nW} \mathrm{cm} \mathrm{cm}^{-2}$ at a $\Delta T$ of $50 \mathrm{~K}$. Even, after 1000 bending cycles, the performance of the device remained unaltered. Erden et al. ${ }^{158}$ reported a ternary hybrid nanocomposite of $\mathrm{TiO}_{2} / \mathrm{CNT} / \mathrm{PANI}$, where the Seebeck coefficient of the binary CNT/PANI composite could be increased by integrating $\mathrm{TiO}_{2}$ nanoparticles into it. The ternary nanocomposites provided two interfaces of $\mathrm{TiO}_{2} / \mathrm{a}-\mathrm{CNT}$ and $\mathrm{TiO}_{2} / \mathrm{PANI}$ for the scattering of low energy carriers, which helped in lowering the thermal conductivity and increasing the thermopower of the composite. The binary a-CNT/PANI composites were formulated by in situ polymerization of aniline in $1 \mathrm{M} \mathrm{HCl}$ at $0{ }^{\circ} \mathrm{C}$ with stirring, followed by washing and drying. The prepared samples were de-doped in ammonium solution for $24 \mathrm{~h}$ followed by washing and drying overnight. Again, re-doping was done with CSA in $m$-cresol, and $\mathrm{TiO}_{2}$ (anatase) was added to re-doped samples in varying concentrations and at different temperatures to check the effect on thermoelectric properties. Finally, to prepare hybrid nanocomposite films on a glass substrate, a drop-casting method was used, and the films were air-dried at $40{ }^{\circ} \mathrm{C}$. To further improve the performance of the thermoelectric nanocomposite films, they were water treated and processed at various temperatures. To achieve a better TE performance, the ratio of $\mathrm{TiO}_{2}$ nanoparticles to CNT/PANI in the composite was optimized. Therefore, for an optimized ratio of $30 \% \mathrm{TiO}_{2}$ with $70 \%$ (a-CNT $70 \% / \mathrm{PANI} 30 \%$ ), the achieved power factor, electrical conductivity, and thermopower at a temp of $40{ }^{\circ} \mathrm{C}$ were $114.5 \mu \mathrm{W} \mathrm{mK} \mathrm{m}^{-2}, 2183 \mathrm{~S} \mathrm{~cm}^{-1}$, and $22.9 \mu \mathrm{V} \mathrm{K}^{-1}$, respectively.

$\mathrm{Lu}$ et $a .^{159}$ reported ternary nanocomposite films of $\mathrm{Te} / \mathrm{PEDOT}: \mathrm{PSS} / \mathrm{Cu}_{7} \mathrm{Te}_{4}$ for a flexible TE power generator. Here, the nanocomposite films were prepared by physically mixing 

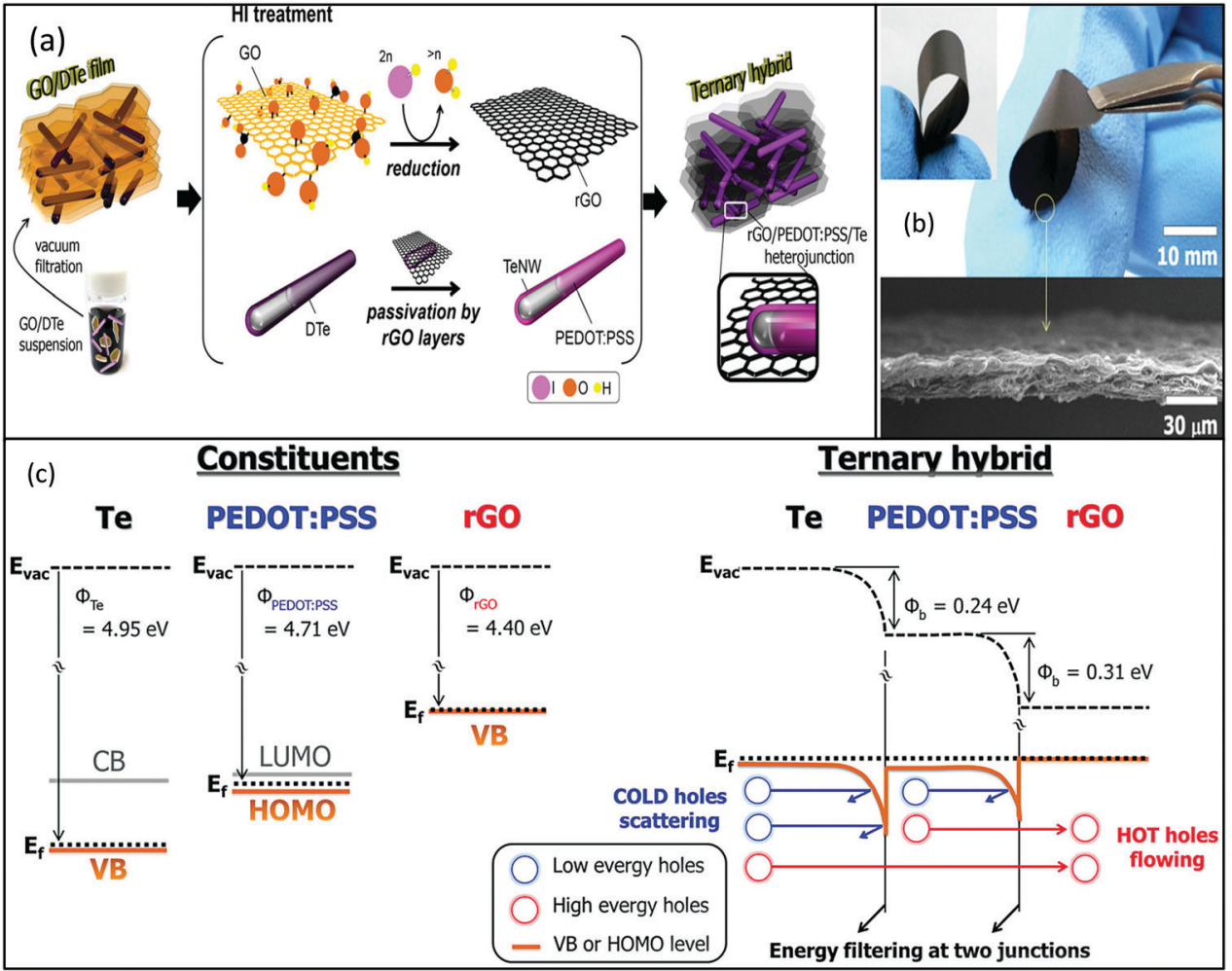

Fig. 13 (a) Synthesis of TE hybrid paper, (b) picture of prepared flexible $\mathrm{G}_{0.1} \mathrm{DT} \mathrm{e}_{0.9}$ hybrid paper, and (c) energy diagram of the rGO/PEDOT:PSS/TeNW heterojunctions showing energy filtering at two junctions: PEDOT:PSS/TeNW and rGO/PEDOT:PSS of DTe. Reprinted with permission from ref. 157 (J. Choi, J. Y. Lee, S. S. Lee, C. R. Park, H. Kim, Adv. Energy Mater. 6 (2016) 1-8). Copyright (2016) John Wiley \& Sons Publications.

the PEDOT:PSS-coated Te nanorod (PC-Te) solution and PEDOT: PSS-coated $\mathrm{Cu}_{7} \mathrm{Te}_{4}$ nanorod (PC-Cu $7 \mathrm{Te}_{4}$ ) solution using a dropcasting method on a glass substrate. The Pc-Te nanorod solution was synthesized by dissolving ascorbic acid in DI water and the addition of PEDOT:PSS to the solution. Then, $\mathrm{Na}_{2} \mathrm{TeO}_{3}$ was slowly added to the vigorously stirring solution followed by heating and natural cooling. The $\mathrm{PC}-\mathrm{Cu}_{7} \mathrm{Te}_{4}$ nanorod solution was synthesized by firstly preparing a PC-Te solution as mentioned above, and then a separate solution of $\mathrm{CuCl}$ in DI water was prepared and poured into the PC-Te solution with vigorous stirring, as shown in Fig. 14. The two heterojunctions of Te/PEDOT:PSS and PEDOT:PSS/ $\mathrm{Cu}_{7} \mathrm{Te}_{4}$ increased the phonon scattering by refining the double carriers, which reduced the thermal conductivity and increased the power factor of the nanocomposite film. The nanocomposite films consisting of $95 \mathrm{wt} \%$ PC-Te nanorods showed a Seebeck coefficient, electrical conductivity, and power factor of $163.5 \mu \mathrm{V} \mathrm{K}^{-1}, 42 \mathrm{~S} \mathrm{~cm}^{-1}$, and $112.3 \mu \mathrm{W} \mathrm{mK}^{-2}$ at $380 \mathrm{~K}$, respectively, which was 5 times higher than a single component. The detailed study of the nanocomposite showed the singlecrystalline nature of Te nanorods, whereas polycrystalline nature of $\mathrm{Cu}_{7} \mathrm{Te}_{4}$. When 8 single legs of TE films were connected in series on a polyimide substrate using silver paste, the produced output voltage, output power, and power density were $31.2 \mathrm{mV}, 94.7 \mathrm{nW}$, and $39.5 \mu \mathrm{W} \mathrm{cm} \mathrm{cm}^{-2}$ at a temperature difference of $39 \mathrm{~K}$, respectively. However, in the bending reliability test after 350 bending cycles, the film resistance increased by a factor of $55 \%$ and became almost constant when subjected to further bending cycles.

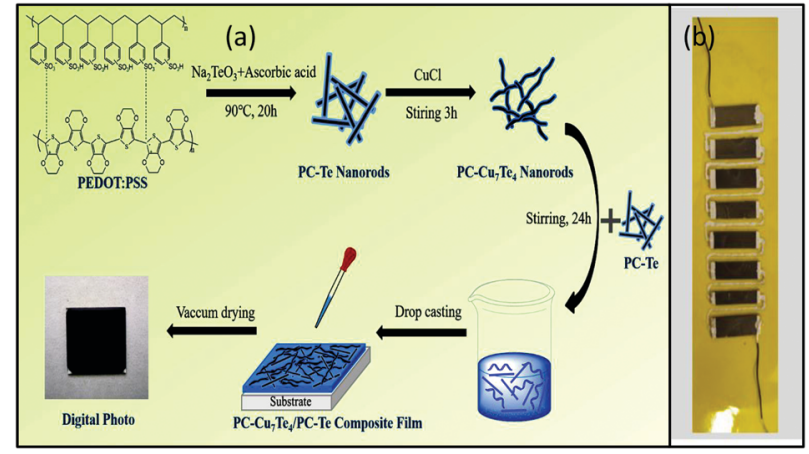

Fig. 14 (a) Schematic of the steps for the fabrication of PC-Cu7Te4/ PC-Te composite film and (b) photograph of a fabricated TE device by using as-prepared film. Reprinted with permission from ref. 159 (Y. Lu, Y. Qiu, Q. Jiang, K. Cai, Y. Du, H. Song, M. Gao, C. Huang, J. He, D. Hu, Appl. Mater. Interfaces. 10 (2018) 42310-42319). Copyright (2018) ACS Publications.

Additionally, after 500 bending cycles, $\mathrm{S}$ decreased by about $21 \%$. The reason for the decrease could be the degradation of the nanorods to nanorod junctions, which affected the performance of the TE. However, the composite showed good mechanical stability in a harsh mechanical environment. $\mathrm{Q}$. Wu and co-workers ${ }^{160}$ reported a unique design of a wearable TEG, in which thermoelectric material-coated yarns were used as the legs and a 3-D fabric as the substrate, as shown in Fig. 15. The p-type thermoelectric yarn was fabricated by 


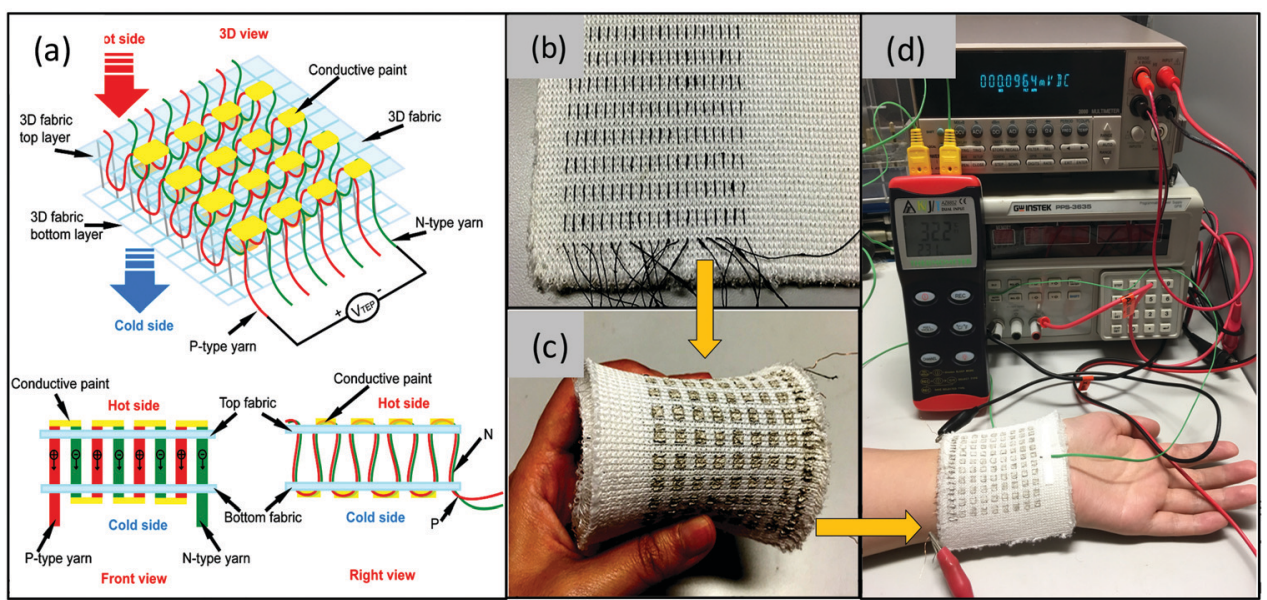

Fig. 15 (a) Systematically structured design of TEG based on 3D fabric, (b) yarn legs embroidered into the spacer fabric matrix, (c) flexibility of the fabricated prototype fabric TEG, (d) image showing the conversion of body heat into output power using the prototype TEG. Reprinted with permission from ref. 160 (Q. Wu, J. Hu, Smart Mater. Struct. 26 (2017) 045037). Copyright (2017), IOP Science Publications.

coating it with a nonionic waterborne polyurethane (NWPU)/ PEDOT:PSS/multi-walled carbon nanotube (MWCNT) composite containing $20 \mathrm{wt} \%$ MWCNTs and 1:4 ratio of MWCNTs to PEDOT:PSS. Similarly, the n-type yarn NWPU/nitrogen-doped multi-walled carbon nanotube (N-MWCNT) composite consisting of $30 \mathrm{wt} \%$ of N-MWCNTs was used as a coating agent. Commercial polyester yarns were directly coated with the composites using a dip-coating method 5 times. After coating the yarn, it was dried in an oven. These composites were synthesized by dispersing MWCNTs/(N-MWCNT) in PEDOT:PSS solutions containing $5 \mathrm{wt} \% \mathrm{DMSO} /$ water, respectively, followed by the addition of NWPU and sonication. The p- and n-type yarns were stitched onto the spacer fabric substrate alternately and serially connected using silver paste. The 3-D fabric generator produced an output voltage of $\sim 800 \mu \mathrm{V}$ and output power of $\sim 2.6 \mathrm{nW}$ at $\Delta T$ of $66 \mathrm{~K}$. This fabric TEG can be used in winter clothing, where a large temperature difference can be maintained between the body skin and ambient air. This device can be easily scalable because of its simple structure and costeffectiveness.

Wang et al. ${ }^{161}$ reported a novel in situ method for synthesizing ternary PANI/SWNT/Te nanocomposites soluble in water, which showed enhanced thermoelectric properties. The PANI/SWNT nanocomposites were synthesized via the in situ polymerization of aniline with ammonium persulfate as an oxidant in the presence of a solution of poly(styrenesulfonic acid) (PSSA) containing SWNTs followed by sonication and vacuum drying. The PANI/SWNT/Te nanocomposite solution was synthesized by dissolving hydroxyl citric acid $\left(\mathrm{C}_{6} \mathrm{H}_{8} \mathrm{O}_{8}\right)$, $\mathrm{Na}_{2} \mathrm{TeO}_{3}$, and PANI/SWNT in different ratios in DI water forming a milky-white bluish solution by stirring at $90{ }^{\circ} \mathrm{C}$ for $15 \mathrm{~h}$. Stable films were fabricated using an optimal concentration of $10 \mathrm{wt} \% \mathrm{Te} / \mathrm{PANI} / \mathrm{SWNT}$ solution. Considering that the PANI coating was formed around SWNTs and Te nanorods, which created two heterojunctions, these two energy filtering junctions of PANI/SWNTs and PANI/Te helped in scattering low energy carriers, resulting in a high Seebeck coefficient of $54 \mu \mathrm{V} \mathrm{K}^{-1}$ and high electrical conductivity of $345 \mathrm{~S} \mathrm{~cm}^{-1}$. The obtained power factor and power density from these ternary nanocomposite films were $101 \mu \mathrm{W} \mathrm{mK} \mathrm{m}^{-2}$ and $62.4 \mu \mathrm{W} \mathrm{cm}{ }^{-2}$, respectively. Wang et al. ${ }^{162}$ reported the synthesis of a ternary nanocomposite of polypyrrole/graphene/polyaniline (PPy/GNs/ PANI) via a solution method and in situ polymerization process. The homogenous dispersion of graphene in the polymer matrix increased the carrier mobility and augmented the nanointerfaces of the composite to scatter low energy carriers. The major ordered structure of the composite was an outcome of the potent $\pi-\pi$ interaction between PPy, GNs, and PANI. However, after cold pressing, enhanced TE properties of the PPy/GNs, PANI/GNs, and PPy/GNs/PANI composites were recorded at different temperatures. Therefore, the resultant superior electrical conductivity, Seebeck coefficient, and higher power factor were due to the synergetic effect of both processes. Further, for the optimal concentration of $32 \mathrm{wt} \%$ graphene, the ternary composite of PPy/GNs/PANI exhibited a high PF of up to $52.5 \mu \mathrm{W} \mathrm{mK} \mathrm{mK}^{-2}$, electrical conductivity reaching $50000 \mathrm{~S} \mathrm{~m}^{-1}$, and Seebeck coefficient of up to $32.4 \mu \mathrm{V} \mathrm{K}^{-1}$. Cho et al. ${ }^{163}$ reported uniformly ordered polyelectrolyte carbon nanocomposites (PCNs) of PANI, graphene, and double-walled carbon nanotubes (DWNT) via the sequential layer deposition technique. As mentioned above, the superior electrical conductivity associated with graphene and the strong $\pi-\pi$ interaction between PANI and DWNT resulted in increased carrier mobility and enhanced thermoelectric properties. Thus, to obtain thermoelectric films, bilayers (BLs) of PANI/graphene and PANI/ DWNT and quad layers (QLs) of PANI/graphene/PANI/DWNT were assembled on an Si-wafer by utilizing the layer-by-layer (LBL) deposition technique. For the LBL deposition technique, the precursors such as cationic PANI having a $\mathrm{pH}$ value of 2.5 , negatively-charged sodium dodecylbenzenesulfonate (SDBS)stabilized DWNT, and poly(4-stryrenesulfonic acid) (PSS)stabilized graphene were used. During the deposition of the 
samples, linear growth with the number of cycles was observed for the PANI/graphene and PANI/DWNT BL films and PANI/ graphene/PANI/DWNT QL films with a mean thickness of $4.8 \mathrm{~nm}$ and $6.3 \mathrm{~nm}$ per $\mathrm{BL}$, and $11.8 \mathrm{~nm}$ per QL, respectively. The determined concentration ratio of QL-PCN was $37.7 \mathrm{wt} \%$ for PANI, $21.4 \mathrm{wt} \%$ for graphene, and $40.9 \mathrm{wt} \%$ for DWNT. At a threshold limit of 40 cycles, the QL films of PANI/graphene/ PANI/DWNT showed the maximum electrical conductivity of $1080 \mathrm{~S} \mathrm{~cm}^{-1}$, Seebeck coefficient of $130 \mu \mathrm{V} \mathrm{K}^{-1}$, and minimum sheet resistance of $19.8 \Omega \mathrm{sq}^{-1}$, respectively. The layer-by-layer (LBL) deposited films of PCN demonstrated a power factor of $1825 \mu \mathrm{W} \mathrm{mK}{ }^{-2}$, which was better than that of lead telluride and more than half the value of bulk $\mathrm{Bi}_{2} \mathrm{Te}_{3}$. The deposited QL films contained many nano-interfaces, which were enough to scatter low energy phonons to reduce the thermal conductivity.

Meng et al. ${ }^{164}$ used a vacuum-assisted filtering method to develop single-walled carbon nanotube (SWCNT)/PEDOT: PSS-coated Te nanorod (PC-Te) composite films. To prepare the PC-Te nanorods, ascorbic acid was dissolved in distilled water with the addition of PEDOT:PSS and $\mathrm{Na}_{2} \mathrm{TeO}_{3}$. The suspension was stirred for $20 \mathrm{~h}$ at a temperature of $90{ }^{\circ} \mathrm{C}$. The precipitate was centrifuged and washed with distilled water until the supernatant became light blue. Then, the SWCNT/ PC-Te nanocomposite solution was prepared by mixing aqueous solutions of both materials in different mass ratios, followed by ultra-sonication. The composite films were obtained via vacuum filtration through a porous nylon membrane filter and drying in a vacuum oven. Later, to boost the thermoelectric properties of the nanocomposite films, they were treated with $\mathrm{H}_{2} \mathrm{SO}_{4}$, and heated and washed with deionized water and ethyl alcohol. The composite films containing $70 \mathrm{wt} \%$ SWCNT gave the highest thermoelectric properties, i.e., Seebeck coefficient of $\sim 48 \mu \mathrm{V} \mathrm{K} \mathrm{K}^{-1}$, electrical conductivity of $361.6 \mathrm{~S} \mathrm{~cm}^{-1}$, and power factor of $79.9 \mu \mathrm{W} \mathrm{mK}^{-2}$. A flexible TEG prototype was fabricated by electrically connecting six strips of composite film in series using silver paste. The obtained power output and voltage were $53.6 \mathrm{nW}$ and $5.6 \mathrm{mV}$ at $\Delta T$ of $44 \mathrm{~K}$, respectively, with a power density of $21.4 \mu \mathrm{W} \mathrm{cm} \mathrm{cm}^{-2}$.

A ternary hybrid nanocomposite of RGO/CdS/PANI using a two-step in situ method was developed by More and coworkers. ${ }^{165}$ In the first step, by varying the concentration of RGO, CdS quantum dots were grown on RGO sheets to form an RGO-CdS nanocomposite, where 2-mercaptopropionic acid was used as the capping agent. The authors reported the in situ polymerization of aniline in the presence of ammonium persulfate as an oxidizing agent to prepare polyaniline (PANI). Here, PANI served as a connecting bridge between the RGO/CdS nanocomposite to increase the carrier mobility. With a $0.4 \mathrm{wt} \%$ RGO loading, the highest obtained Seebeck coefficient, electrical conductivity, thermal conductivity, and power factor were $18 \mu \mathrm{V} \mathrm{K}^{-1}, 2.9 \times 10^{5} \mathrm{~S} \mathrm{~m}^{-1}, 0.14 \mathrm{~W} \mathrm{~m}^{-1} \mathrm{~K}^{-1}$, and $92 \mu \mathrm{W} \mathrm{mK}^{-2}$ $(\mathrm{ZT}=1.97)$, respectively. Additionally, the authors described the possible charge transfer mechanism in the ternary hybrid nanocomposite responsible for its high TE properties, as shown in Fig. 16. Kshirsagar et al. ${ }^{166}$ reported an I/III/V-VI binary metal selenide-MWCNTs-PANI-based nanocomposite for

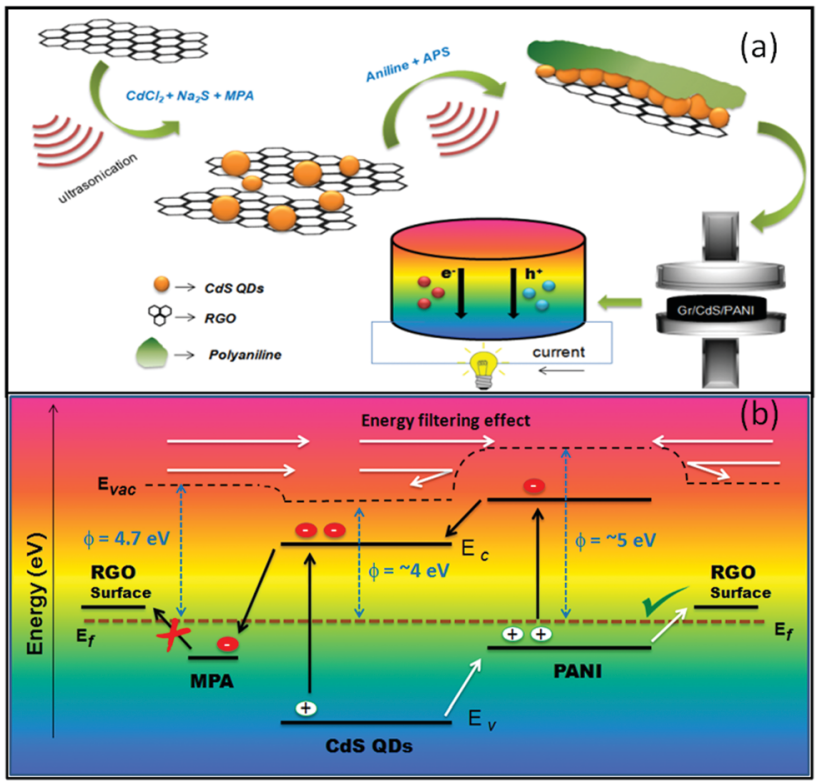

Fig. 16 (a) Schematic representation of the preparation of RGO-CdSPANI nanocomposites for thermoelectric applications and (b) graphical representation of the energy levels of RGO-CdS-PANI nanocomposite promoting $\mathrm{p}$-type charge transport and energy filtering effect. Reproduced from ref. 165 with permission from RSC Publications, Copyright 2017.

thermoelectric applications, which provided good thermoelectric properties at room temperature. Here, an ex situ synthetic method was used to prepare all the ternary hybrid nanocomposites. Nanoparticles of CuSe, $\mathrm{Ag}_{2} \mathrm{Se}, \mathrm{In}_{2} \mathrm{Se}_{3}, \mathrm{Sb}_{2} \mathrm{Se}_{3}$ and PANI were synthesized separately using previously reported methods. Then, the synthesized nanoparticles, PANI, and commercial MWCNTs were mixed properly with the help of a mortar-pestle to form a homogeneous powder. Therefore, the obtained $\mathrm{Ag}_{2}$ Se-MWCNT-PANI (ASCP) nanocomposite showed p-type behavior with a ZT value of 0.012 at room temperature and other thermoelectric properties, i.e., electrical conductivity of $2962 \mathrm{~S} \mathrm{~m}^{-1}$, Seebeck coefficient of $65 \mu \mathrm{V} \mathrm{K} \mathrm{K}^{-1}$, and power factor of $12.5 \mu \mathrm{W} \mathrm{mK}^{-2}$. In contrast, the hybrid nanocomposites of CuSe, $\mathrm{In}_{2} \mathrm{Se}_{3}$, and $\mathrm{Sb}_{2} \mathrm{Se}_{3}$ NPs with MWCNTs and PANI showed n-type behavior. Here, CSCP, ISCP and SSCP showed the electrical conductivity of 4057, 3048, and $4975 \mathrm{~S} \mathrm{~m}^{-1}$, Seebeck coefficient of $-28.1,-24$, and $-10 \mu \mathrm{V} \mathrm{K}^{-1}$, and power factor of 3.6, 1.75 , and $0.49 \mu \mathrm{W} \mathrm{mK}{ }^{-2}$, respectively. Consequently, all the reviewed ternary hybrid nanocomposites provide a new opportunity to synergistically alter the thermoelectric parameters to harvest energy efficiently. The literature reports on ternary hybrid nanocomposite TE materials are presented in Table 4.

\section{Applications of thermoelectric energy}

Over the past few years, it has been estimated that all the primary energy sectors such as nuclear power plants, hydrothermal power plants, thermal power plants, geothermal power 
Table 4 Literature reports of ternary hybrid nanocomposite-based TE materials

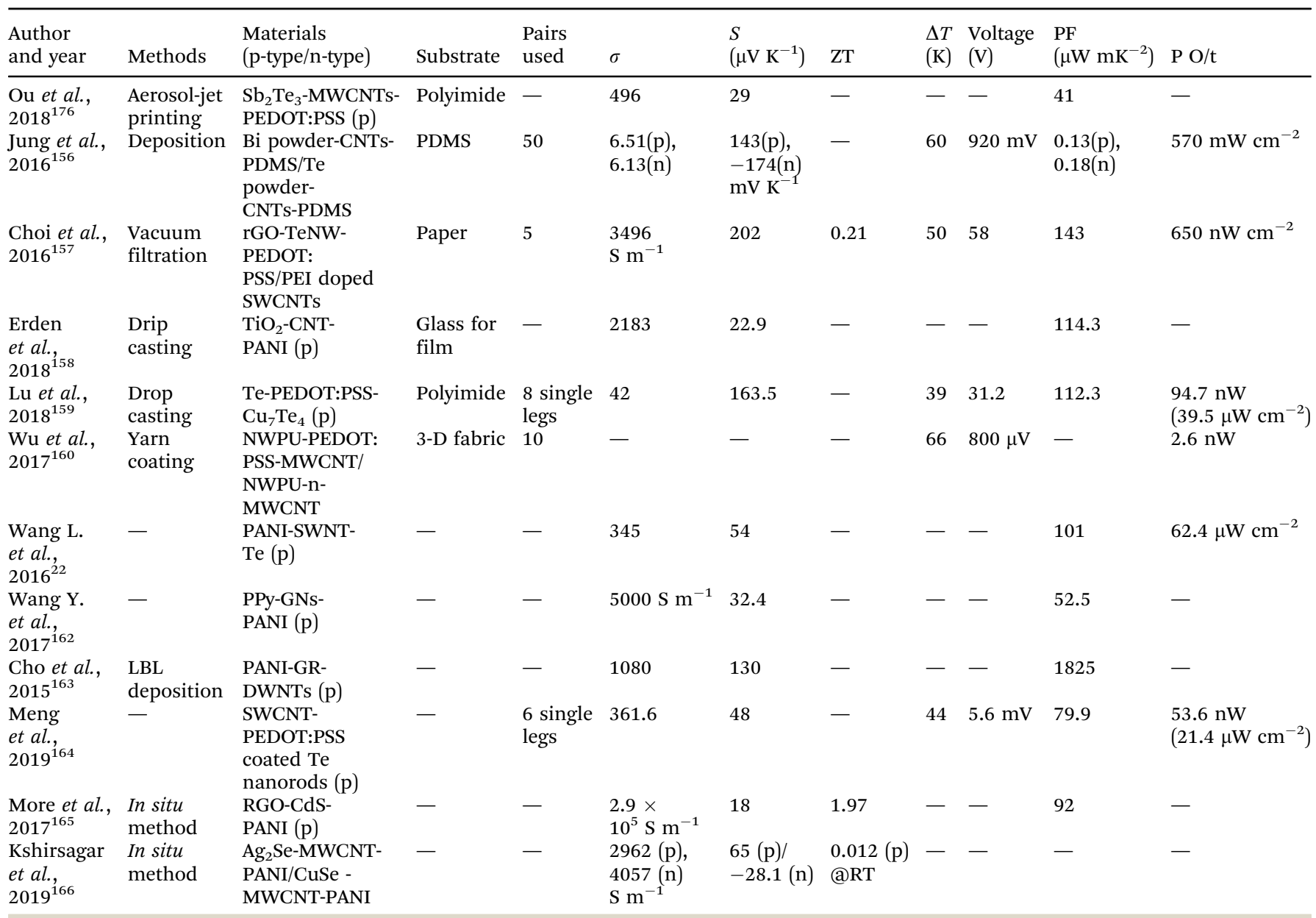

plants, and solar systems utilize only one-third (34\%) of energy effectively and other two-thirds (66\%) of heat is lost to the environment as unusable energy, including in automobiles, residential heating systems and various industrial processes, as shown in Fig. 17. ${ }^{167,168}$ This waste heat can be utilized by thermoelectrics to produce power, which increases the overall efficiency of a plant for power generation. Additionally, this will reduce the warming level of the troposphere by utilizing the lost heat that is transferred to the atmosphere. Thermoelectric devices became popular in 1933 when the US Government Office of Naval Research and Defense Advanced Research Projects Agency asked researchers to find a solution for improving the ZT value. ${ }^{169}$ Thermoelectric materials have been used in deepspace research since 1961. These materials were used in the Apollo mission for providing a long-term energy supply for the first time. In the current scenario, radioisotope-based thermoelectric generators (RTEGs) are becoming the main power source $(350 \mathrm{~W})$ in deep-space missions beyond Mars, where solar cells are not sufficient to supply power. ${ }^{170,171}$

Near two decades ago in 1997, the Cassini Satellite was launched to Saturn with three RETGs, where Pu 238 was used as the thermal energy resource and SiGe as the TE material. In 1920, the Russians cited the first thermoelectric-powered radio systems. In 1954, Chrysler Automobile showed the scope of thermoelectric climate control system technology in luxury cars, in which various thermoelectric-based climate control seats can be used as seat coolers and seat warmers. In addition, nowadays, thermoelectric coolers are becoming attractive for refrigeration purposes. Currently, researchers are focusing on the fabrication of wearable thermoelectric generators for wristwatches, bio-thermoelectric pace-makers, ECG, etc., which can be powered by the temperature difference between the body and its surrounding. ${ }^{2}$ In this regard, textile materials are widely being used as flexible substrates in TE devices because they possess excellent wearability and air-permeability. The continuous demand for wearable and flexible devices has caused researchers to focus on textile-based TE materials. Textile-based flexible TEGs can be used for hotter regions, where generally, the surrounding temperature is higher than the average temperature of the human body. These textile-based TEGs can provide a cool microclimate environment to persons working daily under high temperature conditions such as coal miners, metal workers, and refectory workers to prevent them from heat strokes. ${ }^{172}$ Interestingly, TE materials can also be utilized as a readily available self-powered temperature sensor, where the driving force for temperature sensing can be acquired by the working environment. 
Factories e.g. Metals, Glass,

Polymers etc

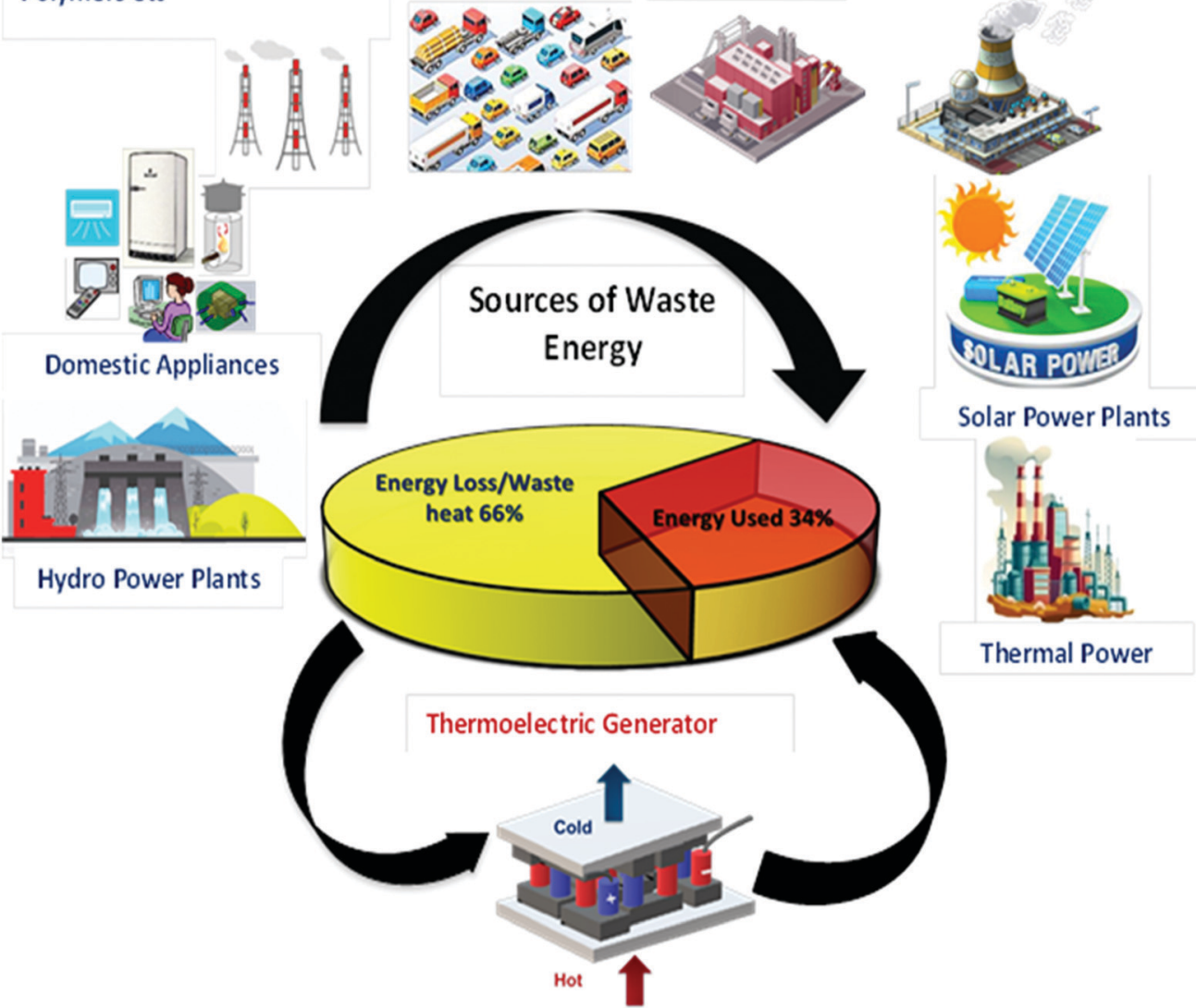

Fig. 17 Schematic of the various types of waste energy sources and utilization of waste heat energy by a thermoelectric generator.

Currently, thermoelectric power generators are already being utilized by remote "self-powered" systems for wireless data communications in the microwatt power range and for automotive systems and deep space missions in the intermediate range of hundreds of watts. However, scientists are still trying to convert industrial waste heat to several kilowatts of power for environmental protection. ${ }^{173}$ Hence, TEGs are a clean energy source studied in current research efforts. The recent advancement in nanotechnology has achieved a significant increment in the efficacy of TE materials for generating power, heating and cooling applications. However, due to their limited efficiency of about 5-6\%, these materials are still developing and not yet suitable for competing with other electrical energy generation technologies in the commercial market. ${ }^{60,174}$ Some of the applications of rigid and wearable TE generators based on their working temperature range are given below.

\subsection{Automobile industry}

The automobile industry is the largest industry in which thermoelectric generators can be potentially used. Only $25 \%$ of the energy stored in gasoline is used in a moving vehicle, and the rest of the energy is lost to the environment through the vehicle exhaust system and radiator. The waste heat produced from the vehicle exhaust system falls normally in a wide temperature range from $100{ }^{\circ} \mathrm{C}-800{ }^{\circ} \mathrm{C}$, which depends on the driving conditions. Thus, to develop TEGs for vehicle heat engines, appropriate aspects should be included such as (1) varying the thermal stresses caused by various driving conditions such as braking, acceleration, stopping and different road conditions such as steep and rough, (2) wide temperature range sustaining TE materials, (3) moving state of TEGs, and (4) exhaust temperature dependence on the consumption of different types of fuel. ${ }^{175}$

The main attraction of using this technology in automobiles is the minimization of fuel consumption and protection of the environment. For the first time in 1914, TEGs were used in vehicles with a reciprocating heat engine to recover some of the waste heat, which was patented by Creveling JL (US). ${ }^{177}$ The hot exhaust gases from the engine were used as a heat source, whereas the circulated cooling water functioned as a heat sink. The applications of thermoelectric generators in automobiles have been deeply investigated for over 90 years to recover waste heat from engines. ${ }^{178,179}$ Some companies developed TEGs that can produce $0.5-1 \mathrm{~kW}$ of electrical energy from the waste of a vehicle heat engine with an overall efficiency of $5-1 \%{ }^{180,181}$ The BMW Group presented a typical photograph of a car with a typical arrangement of a rigid thermoelectric generator, as shown in Fig. 18. ${ }^{182}$ 


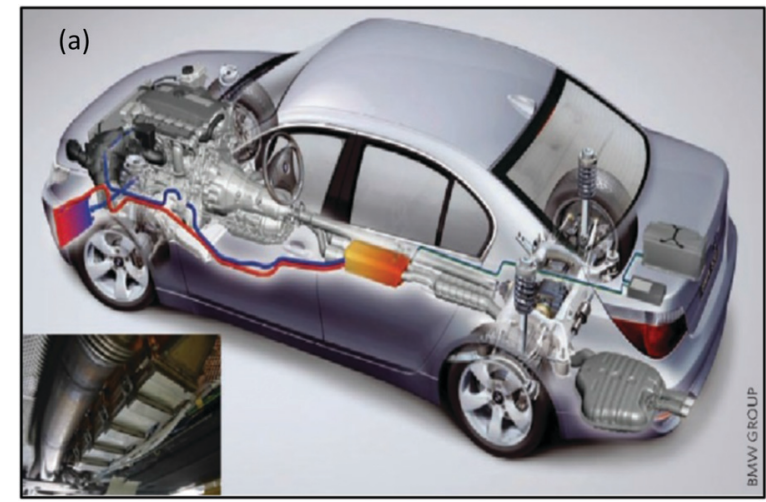

Fig. 18 Integration of thermoelectrics into vehicles for improved fuel efficiency. The image is a BMW 530i concept car with a thermoelectric generator (yellow; and inset) and radiator (red/blue). Reprinted by permission from (Springer Nature), (Nat. Mater.) ref. 182 (An inconvenient truth about thermoelectrics, C. B. Vining, 8 (2009) 83-85), Copyright 2009.

Additionally, to provide comfort zone to passengers, Amerigon Incorporated represented a schematic of car seats integrated with TE devices for climate control. ${ }^{183}$ We know that thermoelectric refrigeration is less efficient than mechanical refrigeration in automobiles, but it still can maintain parts, noise and working fluid, which results in leakage problems.

These devices can also provide a long working life by carefully reducing the efficiency to the very low cooling power of the order of milli-watts. They can be operated easily without any moving device process design and construction. Currently, TE devices perform the main role in the heating ventilation and air conditioning (HVAC) system of vehicles to provide simultaneous heating and cooling by simply reversing the current. During heating mode, the TE HVAC system shows a COP of 2-3 without any subsequent energy consumption procedures, whereas the traditional HVAC system requires additional pump to extract the thermal energy into the working fluid and transfer it through the heat exchanger to other components with a lower COP.

These TE devices in compact form can be inserted into the seats, floor, back of the front seat, dashboard and overhead space of the driver. These installed devices will be used only to cool or heat a particular person, not the whole cabin. The current air conditioner available in the vehicle needs 3500$4000 \mathrm{~W}$ of power to cool the cabin, whereas a thermoelectric cooler (TEC) needs less than $700 \mathrm{~W}$ to cool the driver seat. It can also be activated remotely at a distance of $50 \mathrm{~m}$ or more. This technology has been used by several car manufacturing companies including Ford, GM, Jaguar, Range Rover, Toyota, Hyundai, and Nissan. ${ }^{183}$ Recently, flexible TE devices based on polymers were used on the radiator and exhaust pipe in cars, where the temperature can reach up to $500{ }^{\circ} \mathrm{C}$ and $100{ }^{\circ} \mathrm{C}$, respectively. However, the stability of polymers is challenging for high temperature operations. ${ }^{184}$ Therefore, textile-based TEs as flexible devices can be installed on pipes or radiators regardless of their irregular surface and size. Thus, a large amount of waste heat can be utilized using textile-based TEGs. ${ }^{185}$
Moreover, the use of thermoelectric materials for heating/cooling in vehicles can improve the fuel economy, reduce the toxic and greenhouse gas emission, lower volume, and reduce cost, with greater availability for other applications. The advantages associated with TEGs can achieve significant enhancement in the development of more efficient thermoelectric materials, devices and commercially executable fabrication techniques.

\subsection{Aerospace industry}

The aerospace industry is one of the biggest and most expensive industries, where a continuous supply of electrical power is required for excellent performance. Deployment of satellites in Earth's orbit for exploring the marine life, geography of the Earth, terrestrial surveillance systems, and communication systems require long-life sources of electricity. In addition, the long space missions need a better source of power, which can run up to several years without being damaged in harsh conditions. Thus, thermoelectric generators can be a suitable solution for all these applications due to their good adaptability for various types of heat sources, no movable parts, and no position dependency. TEGs can provide a larger steady-state operation time equal to $10^{5} \mathrm{~h}$, and accurate temperature control within the system. NASA (National Aeronautics and Space Administration) in the USA used high-performance radioisotope generators (RTGs) for the first time in 1961 to provide electrical supply to a spacecraft. Also, TEGs can be used in greater numbers for various types of space missions. RTGs have been used in deep space missions for several years, but the changing scenario of small light-weight, and compact spacecraft require the development of new advanced power sources. Nowadays, thin-film technology-based TEGs are gaining popularity because of their high performance. These thin films can be fabricated by combining semiconductor technology, which is suitable for developing ultra-lightweight thin film TE compact devices to generate the maximum voltage with excellent performance. The superiority of using TEGs in space missions can be seen in various examples such as the Voyager spacecraft with Voyager 1 fleeting into the Helio sheath around 8.3 billion miles from Earth on May 24th, 2006. The successful application also goes to TAGS-85, which has been used in numerous space and terrestrial applications. ${ }^{186}$ Other TEG applications include longlasting power to Viking Landers 1 and 2 and devices that are used in inaccessible or hostile areas such as meteorological data collection and transmission system in off-coast areas and seismic detectors in Alaska. Additionally, during extravehicular activities in space missions, astronauts wear hermetical space suits, where the temperature of outer space varies between $-233-212{ }^{\circ} \mathrm{C}$. ${ }^{187}$ Traditionally, thermal control in a space suit is maintained by liquid cooling and ventilation, which results in bad air permeability of the garments due to the installation of a ventilation unit over the garment. ${ }^{188}$ Therefore, the combination of textile-based thermoelectric generators and coolers can modify current space suit issues such as thermal and moisture comfort of astronauts. When the outer space temperature is greater than the body temperature, TEGs will supply electrical power for supporting the operation of TE coolers. ${ }^{189}$ The aircraft industry, which 
includes both commercial and military aircrafts, can use thermoelectric devices to capture waste heat produced from the engine and supply the generated electric power to the whole aircraft system without affecting the engine performance. This will cut down fuel consumption and ultimately reduce the cost to passengers and cargo airlines. ${ }^{190}$

\subsection{Electronic devices}

Considering the shorter service life of portable batteries, the scientific community is keen to develop low-cost solutions of portable power generators for autonomous energy and wearable biometric monitoring sensors, which can extract energy from different waste heat resources and convert it to useful electricity. Hence, the technological transformation of these advanced microsystems onto flexible substrates can lead to low cost, unobtrusive, and wearable omnipresent integrated devices for monitoring health and biometric parameters. ${ }^{191}$ Many electronic devices such as power amplifiers and microprocessors are operated at high temperatures, which affect the performance, reliability, and lifetime of the device. Thus, to overcome these issues, thermoelectric coolers (TEC) can be utilized as cooling media to eliminate the generated excess heat during the process. Conventional cooling devices are not feasible here due to the requirement of a large cooling area. TECs can couple with the surface of electronic devices to provide effective local cooling. TECs do not make any noise because they have no moving parts. Min et al. ${ }^{192}$ showed an electronic component of infrared arrays integrated with thermoelectric micro-coolers to lower the thermal noise and current leakage produced from an electronic device. Bale et al. ${ }^{193}$ showed the cooling of CdZnTe detector between temperatures of -30 to $-40{ }^{\circ} \mathrm{C}$, which is used in X-ray astronomy. The cooling of the detector can minimize the current leakage and permit the usage of a pulsed reset preamplifier and long pulse shaping times, which significantly enhance the energy solution. For this small application, only $3 \mathrm{~W}$ of electrical power was used. Thermoelectric coolers/generators can also be used in conjugation with solar photovoltaics (PV) to cool or generate electrical power from them, respectively. To obtain the maximum performance from solar PV and to reduce the cost of the panel, thermoelectric coolers should be used to cool the solar PV rather than recovering heat through a thermoelectric generator. ${ }^{194}$ In addition, several attempts were made to attach Peltier modules in residential doors and windows to generate warmer air inside homes in colder regions, although the heat supply was not enough to keep the residential space warm. Presently, e-textiles based on the conducting polymer PEDOT: PSS are getting popular among wearable TEGs. A fabricated 3-D textile as an out-of-plane TE device generated a power output of $1.2 \mu \mathrm{W}$ at a $\Delta T$ of $65 \mathrm{~K}$, which can be used in biomedical devices. ${ }^{195}$

\subsection{Biomedical industry}

In the medical industry, refrigeration of medicine, blood serum, vaccines, and other biological products is necessary to prevent degradation. The properties of these products will only be stabilized in a certain temperature range for excellent results. Here, thermoelectric coolers can be used as a microprocessor-based controlled portable-type medical cooling kit for protecting medical products during storage conditions and freightage, which require $12 \mathrm{~V}$ and $4 \mathrm{~A}$ DC for a smooth operation. ${ }^{196}$ In remote areas of developing countries, the onsite medical services can be provided with sufficient medicines by these TE refrigerators. In addition, doctors can deal with emergency issues by getting the right vaccines on time. TE refrigerators can also work in the food industry where food products need a certain temperature during transportation and storage to preserve their shelf life. Flexible or wearable types of thermoelectric generators (TEGs) can be used in medical devices to provide real-time monitoring of patient health. These wearable devices can be easily wrapped and bent onto the curved skin surface to utilize a large amount of body heat as an energy source. By using a large surface area TEG, the contact between the TEG and skin surface can be enhanced to produce more power. This increased surface area of TEGs can extinguish the necessity of a boost converter. Several small lightweight TEG-based medical sensors with wireless communication can be attached to a patient's body to form a wireless body area network (WBAN), which can provide remote health care services. ${ }^{197,198}$ These devices can perform health monitoring activities such as EEG, ECG, blood pressure ${ }^{85}$ respiration rate, ${ }^{199}$ heart rate, pulse oximetry, ${ }^{200,201}$ body temperature, and sleep period (actigraphy) ${ }^{202}$ at different locations on the wearer's body. ${ }^{203}$ The data collected by medical sensors can be transmitted through various short-range wireless communication protocols such as ANT, Bluetooth, and Zigbee, and collected on a personal server such as a cell phone. Then, health service providers can collect this data from the internet to provide real-time health monitoring. Therefore, integration of short-range wireless communication technology with TEGbased medical sensors can provide efficient and ultra-low power consumption devices. Dementyev et al. reported a Bluetooth low energy (BLE) wireless technique that requires only $35 \mu \mathrm{W}$ at $3.3 \mathrm{~V}$ supply in $120 \mathrm{~s}$ transmission intervals. ${ }^{204}$ Recently, Rose et al. ${ }^{205}$ developed a band-type wearable sensor for sensing heat stress and hydration through the electrolytes present in the wearer's sweat by using battery-free passive radio frequency identification (RFID) and paper microfluidic technology. Further, the technological advancement in integrated circuits, wireless communications, and medical sensors has enabled the fabrication of miniaturized, light-weight, ultra-low powering, and wearable health monitoring devices.

\section{Conclusion and future scope}

It is understood that the traditional inorganic TE materials deliver high output but possesses poor processability, high cost, and severe toxicity. In comparison, organic-based TE materials such as conducting polymers can be advantageous in terms of low-cost, environmentally friendly nature, and low intrinsic thermal conductivity. However, the reported TE 
performances of organic TE materials are relatively low, and thus, researchers have combined them with other semiconducting materials to develop better performing hybrid nanocomposites. These hybrid nanocomposites showed drastic improvements in Seebeck coefficient, power factor, and ZT values owing to the resultant "band engineering" and energy filtering effect. The formation of extra heterojunctions within the hybrid TE materials leads to scattering of low energy charge carriers in favoured directions to give an energy filtering effect. The alignment of energy levels of these component materials may result in the selective transfer of charges, delivering either $\mathrm{p}$ - or n-type behaviour for the entire hybrid nanocomposite. The use of ternary systems allows researchers to overcome the disadvantages of single components to achieve better TE performances. However, for practical applications, the development of outstanding TE performing materials is a persisting challenge as better control of charge carrier in these composites remains elusive. The use of common conducting polymers limits the high-temperature TE applications of these hybrid nanocomposites as the thermal stability of conducting polymers is limited. Additionally, the cost of graphene CNTs and other semiconductor nanoparticles still remains relatively high, creating another issue in their technological transfer. Thus, effective synthetic methods are needed to develop hybrid nanocomposites with controlled structures and refined heterojunctions for better TE efficiency. In terms of thermoelectric devices, their performances can be enhanced in various ways as follows:

1. Improving the properties of thermoelectric materials by computational screening. Here, the synthesis of nanoscale materials, doping of inorganic/organic materials, and formation of hybrid composites are very promising routes to improve the $\mathrm{TE}$ conversion efficiency.

2. Thermal matching of materials, the effect of heat transfer law, the temperature dependency of the material properties, and the greater temperature difference between the two sides of the TE device are possible routes for improvement.

3. Geometrical design including leg length, the thickness of the generator, and the size of hot and cold side substrate has a strong impact on the efficiency of TEGs. Contact quality and resistance also become important parameters for improvement.

4. Fixing the working temperature range for TE materials by enforcing potential heat dissipaters on the cold side.

5. Design optimization of device structure through computer simulations, including the arrangement of the TE elements and device to amend the improved packaging technology for TEGs.

\section{Conflicts of interest}

The authors declare no conflict of interest.

\section{Acknowledgements}

The authors thank the Vice-Chancellor, Defence Institute of Advanced Technology (DIAT), Girinagar, Pune for encouragement.
PKK thanks DMSRDE (DRDO) Kanpur and DIAT, Pune India for the support. Neha thanks Priyanka for fruitful discussion.

\section{Notes and references}

1 S. Patidar, International Journal for Research in Applied Science \& Engineering Technology, 2018, 6, 1992-1996, DOI: $10.22214 /$ ijraset.2018.5325.

2 J. T. Jarman, E. E. Khalil and E. Khalaf, Open Journal of Energy Efficiency, 2013, 2, 143-153.

3 R. Ahiska and H. Mamur, Int. J. Renewable Energy Res., 2014, 4, 128-136.

4 X. Qiu, Western Reverse University, Cleveland, 2008.

5 D. E. Demirocak, Middle East Technical University, Ankara, 2008.

6 Y. Gelbstein, Thermoelectric Energy Conversion: Basic Concepts and Device Applications, 2017, vol. 1, pp. 1-13.

7 G. J. Snyder and E. S. Toberer, Nat. Mater., 2008, 7, 105-114.

8 ed. D. M. Rowe, Thermoelectrics Handbook, CRC Press, 2018.

9 C. B. Vining, W. Laskow, J. O. Hanson, R. R. Van der Beck and P. D. Gorsuch, J. Appl. Phys., 1991, 69, 4333-4340.

10 S. R. Brown, S. M. Kauzlarich, F. Gascoin and G. J. Snyder, Chem. Mater., 2006, 18, 1873-1877.

11 N. V. Nong and L. T. Hung, Thermoelectric Energy Conversion, Basic Concepts and Device Applications, 2017, vol. 1, pp. 15-35.

12 R. Venkatasubramanian, E. Siivola, T. Colpitts, K. The, G. Kanatzidis, T. Zt and B. Vining, Nature, 2001, 413, 597-602.

13 Y. Ma, G. Chen, M. S. Dresselhaus, B. Poudel, B. Yu, D. Vashaee, D. Wang, X. Yan, Z. Ren, A. Minnich, A. Muto, X. Chen, J. Liu, Q. Hao and Y. Lan, Science, 2008, 320, 634-638.

14 T. C. Harman, M. P. Walsh, B. E. Laforge and G. W. Turner, J. Electron. Mater., 2005, 34, L19-L22.

15 Y. Pei, X. Shi, A. Lalonde, H. Wang, L. Chen and G. J. Snyder, Nature, 2011, 473, 66-69.

16 R. Amatya and R. J. Ram, J. Electron. Mater., 2012, 41, 1011-1019.

17 G. J. Snyder, Int. Conf. Thermoelectr. ICT, Proc., 2008, 2002-Janua, 424-426.

18 Y. Du, J. Xu, B. Paul and P. Eklund, Appl. Mater. Today, 2018, 12, 366-388.

19 J. Yang, H. L. Yip and A. K. Y. Jen, Adv. Energy Mater., 2013, 3, 549-565.

20 J. Choi, Y. Jung, S. J. Yang, J. Y. Oh, J. Oh, K. Jo, J. G. Son, S. E. Moon, C. R. Park and H. Kim, ACS Nano, 2017, 11, 7608-7614.

21 M. S. Dresselhaus, G. Chen, M. Y. Tang, R. Yang, H. Lee, D. Wang, Z. Ren, J. P. Fleurial and P. Gogna, Adv. Mater., 2007, 19, 1043-1053.

22 L. Wang, Y. Liu, Z. Zhang, B. Wang, J. Qiu, D. Hui and S. Wang, Composites, Part B, 2017, 122, 145-155.

23 G.-H. Kim, L. Shao, K. Zhang and K. P. Pipe, Nat. Mater., 2013, 12, 719-723. 
24 C. Ou, A. L. Sangle, T. Chalklen, Q. Jing, V. Narayan and S. Kar-Narayan, APL Mater., 2018, 6, 096101.

25 C. Ou, A. L. Sangle, A. Datta, Q. Jing, T. Busolo, T. Chalklen, V. Narayan and S. Kar-Narayan, ACS Appl. Mater. Interfaces, 2018, 10, 19580-19587.

26 O. Bubnova and X. Crispin, Energy Environ. Sci., 2012, 5, 9345-9362.

27 Q. Wei, M. Mukaida, K. Kirihara, Y. Naitoh and T. Ishida, RSC Adv., 2014, 4, 28802-28806.

28 S. J. Kim, J. H. We and B. J. Cho, Energy Environ. Sci., 2014, 7, 1959-1965.

29 O. Bubnova, Z. U. Khan, A. Malti, S. Braun, M. Fahlman, M. Berggren and X. Crispin, Nat. Mater., 2011, 10, 429-433.

30 Z. Lu, M. Layani, X. Zhao, L. P. Tan, T. Sun, S. Fan, Q. Yan, S. Magdassi and H. H. Hng, Small, 2014, 10, 3551-3554.

31 G. H. Kim, L. Shao, K. Zhang and K. P. Pipe, Nat. Mater., 2013, 12, 719-723.

32 E. Destouesse, M. Top, J. Lamminaho, H.-G. Rubahn, J. Fahlteich and M. Madsen, Flexible Printed Electron., 2019, 4, 045004.

33 E. Yvenou, M. Sandroni, A. Carella, M. N. Gueye, J. FaureVincent, S. Pouget, R. Demadrille and J. P. Simonato, Mater. Chem. Front., 2020, 4, 2054-2063.

34 A. Chen, D. Madan, P. K. Wright and J. W. Evans, J. Micromech. Microeng., 2011, 21, 104006, DOI: 10.1088/ 0960-1317/21/10/104006.

35 Y. Nogami, H. Kaneko, T. Ishiguro, A. Takahashi, J. Tsukamoto and N. Hosoito, Solid State Commun., 1990, 76, 583-586.

36 Q. Wang, Q. Yao, J. Chang and L. Chen, J. Mater. Chem., 2012, 22, 17612.

37 N. Nandihalli, C.-J. Liu and T. Mori, Nano Energy, 2020, 78, 105186.

38 C.-T. Hsu, D.-J. Yao, K.-J. Ye and B. Yu, J. Renewable Sustainable Energy, 2010, 2, 013105.

39 W. Glatz and C. Hierold, IEEE 20th International Conference on Micro Electro Mechanical Systems (MEMS), Hyogo, Japan, 2007, pp. 89-92, DOI: 10.1109/MEMSYS.2007.4433021.

40 Z. Fan, Y. Zhang, L. Pan, J. Ouyang and Q. Zhang, Renewable Sustainable Energy Rev., 2020, 110448.

41 A. Nozariasbmarz, H. Collins, K. Dsouza, M. H. Polash, M. Hosseini, M. Hyland, J. Liu, A. Malhotra, F. M. Ortiz, F. Mohaddes, V. P. Ramesh, Y. Sargolzaeiaval, N. Snouwaert, M. C. Özturk and D. Vashaee, Appl. Energy, 2020, 258, 114069.

42 J.-H. Bahk, H. Fang, K. Yazawa and A. Shakouri, J. Mater. Chem. C, 2015, 3, 10362-10374.

43 M. T. Dunham, M. T. Barako, S. LeBlanc, M. Asheghi, B. Chen and K. E. Goodson, Energy, 2015, 93, 2006-2017.

44 F. Suarez, A. Nozariasbmarz, D. Vashaee and M. C. Öztürk, Energy Environ. Sci., 2016, 9, 2099-2113.

45 A. Z. Sahin and B. S. Yilbas, Energy Convers. Manage., 2013, 65, 26-32.

46 K. Yazawa and A. Shakouri, Environ. Sci. Technol., 2011, 45, 7548-7553.

47 M. Hyland, H. Hunter, J. Liu, E. Veety and D. Vashaee, Appl. Energy, 2016, 182, 518-524.
48 D. K. Aswal, R. Basu and A. Singh, Energy Convers. Manage, 2016, 114, 50-67.

49 W. Glatz, S. Muntwyler and C. Hierold, Sens. Actuators, A, 2006, 132, 337-345.

50 W. Glatz, E. Schwyter, L. Durrer and C. Hierold, J. Microelectromech. Syst., 2009, 18, 763-772.

51 G. Liang, J. Zhou and X. Huang, Appl. Energy, 2011, 88, 5193-5199.

52 V. Leonov and R. J. M. Vullers, J. Renewable Sustainable Energy, 2009, 1, 062701.

53 V. Leonov, J. Micromech. Microeng., 2011, 21, 125013.

54 V. Leonov and R. J. M. Vullers, J. Electron. Mater., 2009, 38, 1491-1498.

55 K. Purohit, Int. J. Innov. Res. Eng. Manag., 2016, 3, 259-263.

56 S. K. Yee, S. LeBlanc, K. E. Goodson and C. Dames, Energy Environ. Sci., 2013, 6, 2561-2571.

57 M. Lossec, B. Multon, H. Ben Ahmed and C. Goupil, Eur. Phys. J. Appl. Phys., 2010, 52, 11103.

58 L. Francioso, C. De Pascali, I. Farella, C. Martucci, P. Cretì, P. Siciliano and A. Perrone, J. Power Sources, 2011, 196, 3239-3243.

59 Y.-G. Deng and J. Liu, J. Renewable Sustainable Energy, 2009, 1, 052701.

60 S. A. K., Solar energy engineering processes and systems, 2009.

61 Y. Wu, S. W. Finefrock and H. Yang, Nano Energy, 2012, 1, 651-653.

62 S. Twaha, J. Zhu, Y. Yan and B. Li, Renewable Sustainable Energy Rev., 2016, 65, 698-726.

63 H. J. Goldsmid and R. W. Douglas, Br. J. Appl. Phys., 1954, 5, 386-390.

64 Z. Tian, S. Lee and G. Chen, J. Heat Transfer, 2013, 135, 061605.

65 A. F. Ioffe, Physics of Semiconductors, Academic, 1960, pp. 282-282.

66 A. Bulusu and D. G. Walker, Superlattices Microstruct., 2008, 44, 1-36.

67 S. Fan, J. Zhao, J. Guo, Q. Yan, J. Ma and H. H. Hng, Appl. Phys. Lett., 2010, 96, 182104.

68 W. Xie, X. Tang, Y. Yan, Q. Zhang and T. M. Tritt, Appl. Phys. Lett., 2009, 94, 102111.

69 W. Xie, J. He, H. J. Kang, X. Tang, S. Zhu, M. Laver, S. Wang, J. R. D. Copley, C. M. Brown, Q. Zhang and T. M. Tritt, Nano Lett., 2010, 10, 3283-3289.

70 K. T. Kim and G. H. Ha, J. Nanomater., 2013, 2013, 821657, DOI: $10.1155 / 2013 / 821657$.

71 X. Yan, B. Poudel, Y. Ma, W. S. Liu, G. Joshi, H. Wang, Y. Lan, D. Wang, G. Chen and Z. F. Ren, Nano Lett., 2010, 10, 3373-3378.

72 Y. Q. Cao, X. B. Zhao, T. J. Zhu, X. B. Zhang and J. P. Tu, Appl. Phys. Lett., 2008, 92, 143106.

73 Z. Cao, E. Koukharenko, R. N. Torah, J. Tudor and S. P. Beeby, J. Phys. Conf. Ser., 2014, 557, 6-11.

74 M. K. Kim, M. S. Kim, S. Lee, C. Kim and Y. J. Kim, Smart Mater. Struct., 2014, 23, 105002, DOI: 10.1088/0964-1726/ 23/10/105002. 
75 D. Madan, A. Chen, P. K. Wright and J. W. Evans, J. Electron. Mater., 2012, 41, 1481-1486.

76 T. Nishino and T. Suzuki, J. Micromech. Microeng., 2017, 27, 1-8. 77 S. Shin, R. Kumar, J. W. Roh, D. S. Ko, H. S. Kim, S. Il Kim, L. Yin, S. M. Schlossberg, S. Cui, J. M. You, S. Kwon, J. Zheng, J. Wang and R. Chen, Sci. Rep., 2017, 7, 1-9.

78 F. Suarez, D. P. Parekh, C. Ladd, D. Vashaee, M. D. Dickey and M. C. Öztürk, Appl. Energy, 2017, 202, 736-745.

79 P. Sundarraj, D. Maity, S. S. Roy and R. A. Taylor, RSC Adv., 2014, 4, 46860-46874.

80 T. M. Tritt and M. A. Subramanian, MRS Bull., 2006, 31, 188-198.

81 Z. H. Dughaish, Phys. B, 2002, 322, 205-223.

82 H. J. Goldsmid, Introduction to Thermoelectricity, Springer Berlin Heidelberg, Berlin, Heidelberg, 2010, vol. 121.

83 G. Chen, M. S. Dresselhaus, G. Dresselhaus, J.-P. Fleurial and T. Caillat, Int. Mater. Rev., 2003, 48, 45-66.

84 K. Biswas, J. He, I. D. Blum, C.-I. Wu, T. P. Hogan, D. N. Seidman, V. P. Dravid and M. G. Kanatzidis, Nature, 2012, 489, 414-418.

85 K. F. Hsu, S. Loo, F. Guo, W. Chen, J. S. Dyck, C. Uher, T. Hogan, E. K. Polychroniadis and M. G. Kanatzidis, Science, 2004, 303, 818-821.

86 B. A. Cook, M. J. Kramer, J. L. Harringa, M.-K. Han, D.-Y. Chung and M. G. Kanatzidis, Adv. Funct. Mater., 2009, 19, 1254-1259.

87 D. L. Medlin and G. J. Snyder, Curr. Opin. Colloid Interface Sci., 2009, 14, 226-235.

88 J. Androulakis, C.-H. Lin, H.-J. Kong, C. Uher, C.-I. Wu, T. Hogan, B. A. Cook, T. Caillat, K. M. Paraskevopoulos and M. G. Kanatzidis, J. Am. Chem. Soc., 2007, 129, 9780-9788.

89 J. Androulakis, K. F. Hsu, R. Pcionek, H. Kong, C. Uher, J. J. D'Angelo, A. Downey, T. Hogan and M. G. Kanatzidis, Adv. Mater., 2006, 18, 1170-1173.

90 P. F. P. Poudeu, J. D’Angelo, A. D. Downey, J. L. Short, T. P. Hogan and M. G. Kanatzidis, Angew. Chem., Int. Ed., 2006, 118, 3919-3923.

91 S. N. Girard, J. He, X. Zhou, D. Shoemaker, C. M. Jaworski, C. Uher, V. P. Dravid, J. P. Heremans and M. G. Kanatzidis, J. Am. Chem. Soc., 2011, 133, 16588-16597.

92 Q. Zhang, H. Wang, W. Liu, H. Wang, B. Yu, Q. Zhang, Z. Tian, G. Ni, S. Lee, K. Esfarjani, G. Chen and Z. Ren, Energy Environ. Sci., 2012, 5, 5246-5251.

93 H. Wang, Y. Pei, A. D. LaLonde and G. J. Snyder, Adv. Mater., 2011, 23, 1366-1370.

94 H. Wang, Z. M. Gibbs, Y. Takagiwa and G. J. Snyder, Energy Environ. Sci., 2014, 7, 804-811.

95 W. Liu, X. Yan, G. Chen and Z. Ren, Nano Energy, 2012, 1, $42-56$.

96 Thermal Conductivity, ed. T. M. Tritt, Springer, US, 2004.

97 S. Q. Bai, X. Shi and L. D. Chen, Appl. Phys. Lett., 2010, 96, 202102.

98 Z.-G. Chen, G. Han, L. Yang, L. Cheng and J. Zou, Prog. Nat. Sci.: Mater. Int., 2012, 22, 535-549.

99 H. Li, X. Tang, Q. Zhang and C. Uher, Appl. Phys. Lett., 2008, 93, 252109.
100 W. Zhao, P. Wei, Q. Zhang, C. Dong, L. Liu and X. Tang, J. Am. Chem. Soc., 2009, 131, 3713-3720.

101 X. Shi, J. Yang, J. R. Salvador, M. Chi, J. Y. Cho, H. Wang, S. Bai, J. Yang, W. Zhang and L. Chen, J. Am. Chem. Soc., 2012, 134, 2842.

102 G. Rogl, A. Grytsiv, P. Rogl, E. Bauer, M. B. Kerber, M. Zehetbauer and S. Puchegger, Intermetallics, 2010, 18, 2435-2444.

103 W. Xie, A. Weidenkaff, X. Tang, Q. Zhang, J. Poon and T. Tritt, Nanomaterials, 2012, 2, 379-412.

104 G. Joshi, T. Dahal, S. Chen, H. Wang, J. Shiomi, G. Chen and Z. Ren, Nano Energy, 2013, 2, 82-87.

105 X. Yan, W. Liu, H. Wang, S. Chen, J. Shiomi, K. Esfarjani, H. Wang, D. Wang, G. Chen and Z. Ren, Energy Environ. Sci., 2012, 5, 7543.

106 X. W. Wang, H. Lee, Y. C. Lan, G. H. Zhu, G. Joshi, D. Z. Wang, J. Yang, A. J. Muto, M. Y. Tang, J. Klatsky, S. Song, M. S. Dresselhaus, G. Chen and Z. F. Ren, Appl. Phys. Lett., 2008, 93, 193121.

107 G. Joshi, H. Lee, Y. Lan, X. Wang, G. Zhu, D. Wang, R. W. Gould, D. C. Cuff, M. Y. Tang, M. S. Dresselhaus, G. Chen and Z. Ren, Nano Lett., 2008, 8, 4670-4674.

108 M. S. El-Genk, H. H. Saber and T. Caillat, Energy Convers. Manage., 2003, 44, 1755-1772.

109 S. Narazu, Y. Hadano, T. Takabatake and H. Sugawara, J. Phys. Soc. Jpn., 2008, 77, 238-241.

110 J. Grunlan, C. Yu, Y. Kim, K. Choi and D. Kim, ACS Nano, 2010, 4, 513-523.

111 R. S. Lee, H. J. Kim, J. E. Fischer, A. Thess and R. E. Smalley, Nature, 1997, 388, 255-257.

112 Y. Nonoguchi, M. Nakano, T. Murayama, H. Hagino, S. Hama, K. Miyazaki, R. Matsubara, M. Nakamura and T. Kawai, Adv. Funct. Mater., 2016, 26, 3021-3028.

113 C. Yu, A. Murali, K. Choi and Y. Ryu, Energy Environ. Sci., 2012, 5, 9481.

114 Y. Nonoguchi, K. Ohashi, R. Kanazawa, K. Ashiba, K. Hata, T. Nakagawa, C. Adachi, T. Tanase and T. Kawai, Sci. Rep., 2013, 3, 3344 .

115 P. G. Collins, Science, 2000, 287, 1801-1804.

116 C. J. An, Y. H. Kang, H. Song, Y. Jeong and S. Y. Cho, J. Mater. Chem. A, 2017, 5, 15631-15639.

117 S. L. Kim, K. Choi, A. Tazebay and C. Yu, ACS Nano, 2014, 8, 2377-2386.

118 M. Ito, T. Koizumi, H. Kojima, T. Saito and M. Nakamura, J. Mater. Chem. A, 2017, 5, 12068-12072.

119 C. Cho, M. Culebras, K. L. Wallace, Y. Song, K. Holder, J. H. Hsu, C. Yu and J. C. Grunlan, Nano Energy, 2016, 28, 426-432.

120 C. Yu, Y. S. Kim, D. Kim and J. C. Grunlan, Nano Lett., 2009, 9, 1283.

121 L. Vaisman, H. D. Wagner and G. Marom, Adv. Colloid Interface Sci., 2006, 128-130, 37-46.

122 R. Bandyopadhyaya, E. Nativ-Roth, O. Regev and R. Yerushalmi-Rozen, Nano Lett., 2002, 2, 25-28.

123 E. Kymakis and G. A. J. Amaratunga, J. Appl. Phys., 2006, 99, 084302 . 
124 M. C. Hermant, B. Klumperman, A. V. Kyrylyuk, P. van der Schoot and C. E. Koning, Soft Matter, 2009, 5, 878.

125 N. R. Tummala and A. Striolo, ACS Nano, 2009, 3, 595-602.

126 H. Wang, W. Zhou, D. L. Ho, K. I. Winey, J. E. Fischer, C. J. Glinka and E. K. Hobbie, Nano Lett., 2004, 4, 1789-1793.

127 L. Liu and J. C. Grunlan, Adv. Funct. Mater., 2007, 17, 2343-2348.

128 Y.-C. Tsai, C.-C. Chiu, M.-C. Tsai, J.-Y. Wu, T.-F. Tseng, T.-M. Wu and S.-F. Hsu, Carbon, 2007, 45, 2823-2827.

129 J. C. Grunlan, L. Liu and O. Regev, J. Colloid Interface Sci., 2008, 317, 346-349.

130 Y. Dror, W. Pyckhout-Hintzen and Y. Cohen, Macromolecules, 2005, 38, 7828-7836.

131 R. Haggenmueller, S. S. Rahatekar, J. A. Fagan, J. Chun, M. L. Becker, R. R. Naik, T. Krauss, L. Carlson, J. F. Kadla, P. C. Trulove, D. F. Fox, H. C. DeLong, Z. Fang, S. O. Kelley and J. W. Gilman, Langmuir, 2008, 24, 5070-5078.

132 M. Zheng, A. Jagota, E. D. Semke, B. A. Diner, R. S. Mclean, S. R. Lustig, R. E. Richardson and N. G. Tassi, Nat. Mater., 2003, 2, 338-342.

133 J. Y. Kim, J. H. Jung, D. E. Lee and J. Joo, Synth. Met., 2002, 126, 311-316.

134 C. Meng, C. Liu and S. Fan, Adv. Mater., 2010, 22, 535-539. 135 Q. Yao, L. Chen, W. Zhang, S. Liufu and X. Chen, ACS Nano, 2010, 4, 2445-2451.

136 C. Yu, K. Choi, L. Yin and J. C. Grunlan, ACS Nano, 2013, 7, 9506.

137 M. Nakano, Y. Nonoguchi, T. Nakashima, K. Hata and T. Kawai, RSC Adv., 2016, 6, 2489-2495.

138 Y. Mamunya, A. Boudenne, N. Lebovka, L. Ibos, Y. Candau and M. Lisunova, Compos. Sci. Technol., 2008, 68, 1981-1988.

139 Y. Agari, A. Ueda, Y. Omura and S. Nagai, Polymer, 1997, 38, 801-807.

140 M. Aghelinejad and S. N. Leung, Materials, 2018, 11, 1-13.

141 K. Suemori, S. Hoshino and T. Kamata, Appl. Phys. Lett., 2013, 103, 1-4, DOI: 10.1063/1.4824648.

142 C. A. Hewitt, A. B. Kaiser, S. Roth, M. Craps, R. Czerw and D. L. Carroll, Nano Lett., 2012, 12, 1307-1310.

143 G. H. Kim, D. H. Hwang and S. I. Woo, Phys. Chem. Chem. Phys., 2012, 14, 3530-3536.

144 H. Wang, S. I. Yi, X. Pu and C. Yu, ACS Appl. Mater. Interfaces, 2015, 7, 9589-9597.

145 T. Luo and K. Pan, ACS Appl. Energy Mater., 2018, 1, 1904-1912.

146 J. H. We, S. J. Kim and B. J. Cho, Energy, 2014, 73, 506-512.

147 Y. Yang, Z. H. Lin, T. Hou, F. Zhang and Z. L. Wang, Nano Res., 2012, 5, 888-895.

148 X. Wang, P. Liu, Q. Jiang, W. Zhou, J. Xu, J. Liu, Y. Jia, X. Duan, Y. Liu, Y. Du and F. Jiang, ACS Appl. Mater. Interfaces, 2019, 11, 2408-2417.

149 L. Wang, Z. Zhang, L. Geng, T. Yuan, Y. Liu, J. Guo, L. Fang, J. Qiu and S. Wang, Energy Environ. Sci., 2018, 11, 1307-1317.

150 M. He, F. Qiu and Z. Lin, Energy Environ. Sci., 2013, 6, 1352.
151 N. Xiao, X. Dong, L. Song, D. Liu, Y. Tay, S. Wu, L.-J. Li, Y. Zhao, T. Yu, H. Zhang, W. Huang, H. H. Hng, P. M. Ajayan and Q. Yan, ACS Nano, 2011, 5, 2749-2755.

152 T. O. Poehler and H. E. Katz, Energy Environ. Sci., 2012, 5,8110 .

153 Y. Du, S. Z. Shen, K. Cai and P. S. Casey, Prog. Polym. Sci., 2012, 37, 820-841.

154 A. J. Minnich, M. S. Dresselhaus, Z. F. Ren and G. Chen, Energy Environ. Sci., 2009, 2, 466.

155 J. R. Szczech, J. M. Higgins and S. Jin, J. Mater. Chem., 2011, 21, 4037-4055.

156 K. K. Jung, Y. Jung, C. J. Choi, J. M. Lee and J. S. Ko, Curr. Appl. Phys., 2016, 16, 1442-1448.

157 J. Choi, J. Y. Lee, S. S. Lee, C. R. Park and H. Kim, Adv. Energy Mater., 2016, 6, 1-8.

158 F. Erden, H. Li, X. Wang, F. K. Wang and C. He, Phys. Chem. Chem. Phys., 2018, 20, 9411-9418.

159 Y. Lu, Y. Qiu, Q. Jiang, K. Cai, Y. Du, H. Song, M. Gao, C. Huang, J. He and D. Hu, ACS Appl. Mater. Interfaces, 2018, 10, 42310-42319.

160 Q. Wu and J. Hu, Smart Mater. Struct., 2017, 26, 045037.

161 L. Wang, Q. Yao, W. Shi, S. Qu and L. Chen, Mater. Chem. Front, 2017, 1, 741-748.

162 Y. Wang, J. Yang, L. Wang, K. Du, Q. Yin and Q. Yin, ACS Appl. Mater. Interfaces, 2017, 9, 20124-20131.

163 C. Cho, B. Stevens, J. H. Hsu, R. Bureau, D. A. Hagen, O. Regev, C. Yu and J. C. Grunlan, Adv. Mater., 2015, 27, 2996-3001.

164 Q. Meng, K. Cai, Y. Du and L. Chen, J. Alloys Compd., 2019, 163-169.

165 P. V. More, C. Hiragond, A. Dey and P. K. Khanna, Sustainable Energy Fuels, 2017, 1, 1766-1773.

166 A. S. Kshirsagar, C. Hiragond, A. Dey, P. V. More and P. K. Khanna, ACS Appl. Energy Mater., 2019, 2, 2680-2691.

167 Thermoelectric Nanomaterials, ed. K. Koumoto and T. Mori, Springer Berlin Heidelberg, Berlin, Heidelberg, 2013, vol. 182

168 M. Hamid Elsheikh, D. A. Shnawah, M. F. M. Sabri, S. B. M. Said, M. Haji Hassan, M. B. Ali Bashir and M. Mohamad, Renewable Sustainable Energy Rev., 2014, 30, 337-355.

169 L. E. Bell, Science, 2008, 321, 1457-1461.

170 C. B. Vining, in Proceedings of the 5th European Conference on Thermoelectrics, ECT, Odessa Ukraine, 2007.

171 NASA.

172 A. Myers, R. Hodges and J. S. Jur, MRS Advances, 2016, 1, 2665-2670.

173 S. A. Omer and D. G. Infield, Energy Convers. Manage, 2000, 41, 737-756.

174 D. C. Talele, D. S. Deshmukh and P. M. Solanki, Pratibha: International Journal of Science, Spirituality, Business and Technology, 2015, 3, 26-31.

175 X. F. Zheng, C. X. Liu, Y. Y. Yan and Q. Wang, Renewable Sustainable Energy Rev., 2014, 32, 486-503.

176 C. Ou, A. L. Sangle, T. Chalklen, Q. Jing, V. Narayan and S. Kar-Narayan, APL Mater., 2018, 6, 1-8, DOI: 10.1063/ 1.5043547 . 
177 J. L. Creveling, US Pat., 1914, 1118269.

178 J. Bass, Proceedings of the 13th International Conference on Thermoelectrics, American Institute of Physics, New York, Kansas City USA, 1994.

179 B. L. Embry, Proceedings of the intersociety energy conversion engineering conference, IEEE, Boulder, CO, New York, 1968.

180 K. Matsubara, in Twenty-First International Conference on Thermoelectrics, 2002, Proceedings ICT '02., IEEE, pp. 418-423.

181 D. M. Rowe, Thermoelectrics Handbook: Macro to Nano, 2006, Boca Raton, CRC Press, p. 1008, DOI: 10.1201/ 9781420038903.

182 C. B. Vining, Nat. Mater., 2009, 8, 83-85.

$183 \mathrm{~J}$. Lofy, Proceedings of the 21st IEEE International Conference on Thermoelectrics, ICT'02, Long Beach CA, 2002, pp. 471-476.

184 J. Yang and T. Caillat, MRS Bull., 2006, 31, 224-229.

185 K. Ebrahimi, G. F. Jones and A. S. Fleischer, Renewable Sustainable Energy Rev., 2014, 31, 622-638.

186 M. K. Ewert, in International Solar Energy Conference, ASME, Solar Energy Division, Albuquerque, NM, 1998, pp. $375-382$.

187 K. S. Thomas and H. J. McMann, U. S. Spacesuits, Springer New York, New York, NY, 2012.

188 K. Tanaka, K. Nakamura and T. Katafuchi, Acta Astronaut., 2014, 104, 260-265.

189 L. Zhang, S. Lin, T. Hua, B. Huang, S. Liu and X. Tao, Adv. Energy Mater., 2017, 8, 1-18, DOI: 10.1002/aenm.201700524.

190 D. M. Rowe, Hand Book of Thermoelectrics, FL CRC Press, Baco Raton, 1995.

191 L. Francioso, C. De Pascali, P. Siciliano, A. De Risi, C. Veri and M. Pasca, 5th IEEE Int. Workshop on Advances in Sensors and Interfaces IWASI, Bari, Italy, 2013, pp. 104-107, DOI: 10.1109/IWASI.2013.6576100.

192 G. Min and D. M. Rowe, Solid. State. Electron., 1999, 43, 923-929.

193 G. Bale, A. Holland, P. Seller and B. Lowe, Nucl. Instrum. Methods Phys. Res., Sect. A, 1999, 436, 150-154.

194 E. G. M. Najarian, MSE, 2006, 226.

195 A. Lund, Y. Tian, S. Darabi and C. Müller, J. Power Sources, 2020, 480, 228836.

196 N. F. Güler and R. Ahiska, Appl. Therm. Eng., 2002, 22, 1271-1276.

197 M. V. Bavel, Sens. Transducers J., 2008, 94, 103-115.

198 S. Ullah, H. Higgins, B. Braem, B. Latre, C. Blondia, I. Moerman, S. Saleem, Z. Rahman and K. S. Kwak, J. Med. Syst., 2012, 36, 1065-1094.

199 L. D. Hicks and M. S. Dresselhaus, Phys. Rev. B: Condens. Matter Mater. Phys., 1993, 47, 12727-12731.

200 G. Chen, in Semiconductors and Semimetals, Elsevier, 2001, vol. 71, pp. 203-259.

201 J. Heremans and C. M. Thrush, Phys. Rev. B: Condens. Matter Mater. Phys., 1999, 59, 12579-12583.

202 J. F. Behnke, A. L. Prieto, A. M. Stacy and T. Sands, in Eighteenth International Conference on Thermoelectrics. Proceedings, ICT'99 (Cat. No. 99TH8407), IEEE, pp. 451-453.

203 L. E. Bell, J. Electron. Mater., 2009, 38, 1344-1349.

204 A. Dementyev, S. Hodges, S. Taylor and J. Smith, in 2013 IEEE International Wireless Symposium (IWS), IEEE, 2013, pp. 1-4.

205 D. P. Rose, M. E. Ratterman, D. K. Griffin, L. Hou, N. KelleyLoughnane, R. R. Naik, J. A. Hagen, I. Papautsky and J. C. Heikenfeld, IEEE Trans. Biomed. Eng., 2015, 62, 1457-1465. 